\title{
Asymptotic Rigidity of Layered Structures and Its Application in Homogenization Theory
}

\author{
FABian ChristowiaK \& CAROLIN KREISBEck@
}

\author{
Communicated by I. FonSECA
}

\begin{abstract}
In the context of elasticity theory, rigidity theorems allow one to derive global properties of a deformation from local ones. This paper presents a new asymptotic version of rigidity, applicable to elastic bodies with sufficiently stiff components arranged into fine parallel layers. We show that strict global constraints of anisotropic nature occur in the limit of vanishing layer thickness, and give a characterization of the class of effective deformations. The optimality of the scaling relation between layer thickness and stiffness is confirmed by suitable bending constructions. Beyond its theoretical interest, this result constitutes a key ingredient for the homogenization of variational problems modeling high-contrast bilayered composite materials, where the common assumption of strict inclusion of one phase in the other is clearly not satisfied. We study a model inspired by hyperelasticity via $\Gamma$-convergence, for which we are able to give an explicit representation of the homogenized limit problem; it turns out to be of integral form with its density corresponding to a cell formula.
\end{abstract}

\section{Introduction}

Rigidity is a prevalent concept in different areas of mathematics. Generally speaking, it refers to powerful statements that allow one to draw far-reaching conclusions from seemingly little information, such as deducing global properties of a function from local ones. A classical result along these lines is often referred to as Liouville's theorem on geometric rigidity, see e.g. [38]. This says that every smooth local isometry of a domain corresponds to a rigid body motion. A generalization to the Sobolev setting is due to ReshetNYAK [53], and states that if $u \in W^{1, p}\left(\Omega ; \mathbb{R}^{n}\right)$ with $\Omega \subset \mathbb{R}^{n}$ a bounded Lipschitz domain and $1<p<\infty$ satisfies

$$
\nabla u \in S O(n)
$$


pointwise almost everywhere in $\Omega$, then $u$ is harmonic and

$$
u(x)=R x+b \text { for } x \in \Omega \text { with } R \in S O(n) \text { and } b \in \mathbb{R}^{n} .
$$

It is not hard to see that if connectedness of the domain fails, then global rigidity is no longer true, as different connected components can then be rotated and translated individually.

Yet, for a domain that has several rigid components arranged into very fine parallel layers (see Fig. 1), global geometric constraints of anisotropic nature occur in the limit of vanishing layer thickness. Since these restrictions become prominent only after a limit passage, we speak of asymptotic rigidity of layered structures. A first rigorous result in this direction can be found in [16] for the special case $n=2$ and $p=2$. There it was proven that, under the assumption of local volume preservation and up to global rotations, only shear deformations aligned with the orientation of the layers can occur as effective deformations.

In this paper, we extend the result of [16] to arbitrary dimensions $n \geqq 2$ and general $1<p<\infty$, and more significantly, relax the assumption of rigid layers by requiring only sufficient stiffness (see Theorem 1.1). Formally, this corresponds to replacing the exact differential inclusion (1.1) by an approximate one, very much like the quantitative rigidity estimate by FRIESECKE et al. [33, Theorem 3.1] generalizes Reshetnyak's theorem. The paper [33] has initiated increased interest in rigidity and its quantification over the last few years, especially among analysts working on variational methods with applications in materials science. For instance, a quantitative version of piecewise rigidity for $S B V$-functions [12] was established in [32], and there is recent work on the rigidity of conformal maps [31], of nongradient fields [50] and of the non-Euclidean setting [45].

To be more precise about our results, some notation on the geometry of bilayered structures is needed. Throughout the manuscript, let $\Omega \subset \mathbb{R}^{n}$ with $n \geqq 2$ be a bounded Lipschitz domain, $\lambda \in(0,1)$, and $Y=(0,1]^{n}$ the periodicity cell. We set

$$
Y_{\text {soft }}=(0,1]^{n-1} \times(0, \lambda) \text { and } Y_{\text {stiff }}=Y \backslash Y_{\text {soft }},
$$

cf. Fig. 1. Without further mention, $Y_{\text {soft }}$ and $Y_{\text {stiff }}$ are identified with their $Y$-periodic extensions. To describe the thickness of two neighboring layers, we introduce a parameter $\varepsilon>0$, which is supposed to be small and captures the length scale of the heterogeneities. The disjoint sets $\varepsilon Y_{\text {stiff }} \cap \Omega$ and $\varepsilon Y_{\text {soft }} \cap \Omega$ partition the domain $\Omega$ into two phases of alternating layers. Notice that the parameter $\lambda$ stands for the relative thickness of the softer components.

Under certain technical assumptions on the domain, in particular, flatness and cross-section connectedness, which are specified in Definitions 3.6 and 3.7, we obtain as our first main result a characterization for the asymptotic behavior of sequences of functions on $\Omega$ whose gradients are increasingly close to $S O(n)$ in $\varepsilon Y_{\text {stiff }} \cap \Omega$ as $\varepsilon \rightarrow 0$.

Theorem 1.1. Let $\Omega \subset \mathbb{R}^{n}$ be a bounded, flat and cross-section connected Lipschitz domain and $1<p<\infty$. 


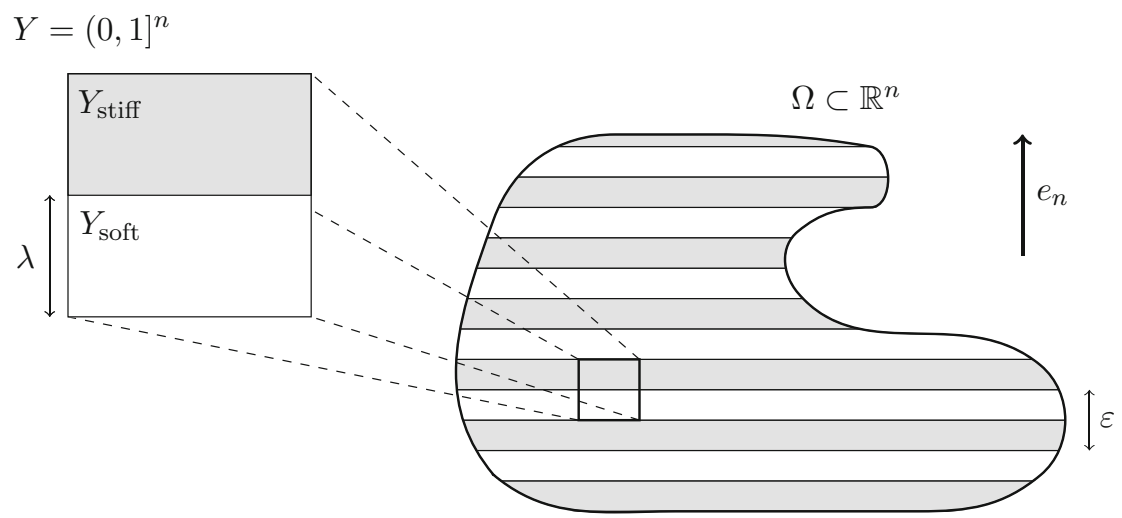

Fig. 1. Illustration of bi-layered structure with stiff (gray) and softer (white) components and periodicity cell $Y$, subdivided into $Y_{\text {soft }}$ and $Y_{\text {stiff }}$

(i) Suppose that $\left(u_{\varepsilon}\right)_{\varepsilon} \subset W^{1, p}\left(\Omega ; \mathbb{R}^{n}\right)$ is such that

$$
\int_{\varepsilon Y_{\text {stiff }} \cap \Omega} \operatorname{dist}^{p}\left(\nabla u_{\varepsilon}, S O(n)\right) \mathrm{d} x \leqq C \varepsilon^{\alpha}
$$

for all $\varepsilon>0$ with $\alpha \geqq 0$ and a constant $C>0$. If $\alpha>p$ and $u_{\varepsilon} \rightarrow u$ in $W^{1, p}\left(\Omega ; \mathbb{R}^{n}\right)$ for some $u \in W^{1, p}\left(\Omega ; \mathbb{R}^{n}\right)$, then

$$
u(x)=R(x) x+b(x), \quad x \in \Omega,
$$

with $R \in W^{1, p}(\Omega ; S O(n))$ and $b \in W^{1, p}\left(\Omega ; \mathbb{R}^{n}\right)$ such that $\partial_{i} R=0$ and $\partial_{i} b=0$ for $i=1, \ldots,(n-1)$.

(ii) If $u \in W^{1, p}\left(\Omega ; \mathbb{R}^{n}\right)$ is of the form (1.4), then there exists a sequence $\left(u_{\varepsilon}\right)_{\varepsilon} \subset$ $W^{1, p}\left(\Omega ; \mathbb{R}^{n}\right)$ such that $u_{\varepsilon} \rightarrow u$ in $W^{1, p}\left(\Omega ; \mathbb{R}^{n}\right)$ and $\nabla u_{\varepsilon} \in S O(n)$ a.e. in $\varepsilon Y_{\text {stiff }} \cap \Omega$ for every $\varepsilon>0$.

One observes that (1.4) resembles (1.2), just that now $R$ will in general not be constant, but depends on the $x_{n}$-variable, and hence, varies in the direction orthogonal to the layers. This condition can be considered the result of a non-trivial interplay between the effects of rigidity and anisotropy.

The proof of Theorem 1.1(i) consists of three main steps: the layerwise approximation of each $u_{\varepsilon}$ by rigid body motions, a compactness argument for the resulting one-dimensional auxiliary functions of piecewise constant rotations, and a limit representation argument. Regarding its overall structure, the reasoning is organized similarly to [16, Proposition 2.1]. Technically, however, the transition from exact to the approximate differential inclusions requires two substantial changes, which make the arguments more involved than in [16]. Instead of Reshetnyak's theorem, we apply the quantitative rigidity estimate on each layer, and the FréchetKolmogorov compactness result (see Lemma 3.4) is used as a refinement of Helly's selection principle.

Proving the second part of Theorem 1.1 involves the explicit construction of an approximating sequence $\left(u_{\varepsilon}\right)_{\varepsilon}$ with the desired properties. To this end, we critically 
exploit the special structure of $u$ as in (1.4), which features a splitting of the $x_{n^{-}}$ variable from the remaining ones, so that $u$ has essentially the character of a onedimensional function.

Remark 1.2. (a) The gradient of $u$ as in (1.4) takes the form

$$
\nabla u=R+\left(\partial_{n} R\right) x \otimes e_{n}+\partial_{n} b \otimes e_{n},
$$

which necessarily requires that $(\nabla u) e_{i}=R e_{i}$ for all $i=1, \ldots, n-1$.

(b) We point out that the scaling regime $\alpha>p$, which quantifies the relation between thickness and stiffness of the layers, is optimal for Theorem 1.1(i). As shown in Section 2, asymptotic rigidity of layered structures fails for $\alpha \leqq p$. We provide explicit examples inspired by bending deformations, for which the limit maps $u$ are such that $\partial_{1} u$ depends non-trivially on $x_{1}$ or $\partial_{1} u$ is not normed to one.

Note that the two extreme cases $\alpha=0$ and " $\alpha=\infty$ " (formal for $\varepsilon^{\alpha}=0$ ) in (1.3) correspond the situations of the stiff layers being actually soft or fully rigid, respectively.

(c) Theorem 1.1 can be extended in different directions. One generalization concerns a $(p, q)$-version Theorem 1.1(i). Indeed, if the exponent $p$ in (1.3) is replaced by $q \in(p, \infty)$ the statement remains valid provided that $\alpha>q$. In this more general setting, we can let $1 \leqq p<\infty$. The only modification in the case $p=1$ is that $R$ and $b$ will be $B V$-functions. We refer to Remark 3.5(a) and Remark 3.2(b) for more details. Moreover, as mentioned in Remark 3.2(c), asymptotic rigidity in the sense of Theorem 1.1(i) still holds if the relative thickness of the stiff layers depend on $\varepsilon$, being much larger than $\varepsilon^{\frac{\alpha}{p}-1}$. For a comment on reduced assumptions for the domain $\Omega$, see Remark 4.2 as well as Theorem 3.1.

(d) If one requires additionally in Theorem 1.1 that the limit function $u$ is locally volume preserving: that is $u \in W^{1, r}\left(\Omega ; \mathbb{R}^{n}\right)$ for $r \geqq n$ with det $\nabla u=1$ a.e. in $\Omega$, then $R e_{n}$ is constant, see Corollary 3.9. In the two-dimensional setting with $n=2$, this implies that $R$ is constant, and one can think of $u$ as horizontal shear deformation up to global rotations, cf. also [16, Proposition 2.1].

From the viewpoint of applications in materials science, Theorem 1.1 identifies characteristics of macroscopically attainable deformations of bi-layered highcontrast composite materials. This observation constitutes an important step towards a rigorous characterization of their effective behavior via homogenization. Indeed, we will discuss in the following how asymptotic rigidity of layered structures serves as the basis for solving a relevant class of homogenization problems in the context of hyperelasticity.

In the 1970s, the Italian school around De Giorgi established the concept of $\Gamma$ convergence $[28,29]$ (see also $[8,26]$ for a comprehensive introduction), which has been used successfully among others in homogenization theory to bridge between microscopic and macroscopic scales. This is a natural notion for variational convergence, i.e. limit passages in parameter-dependent minimization problems. The 
key property is that if a sequence of energy functionals $\Gamma$-converges to a limit functional, this implies, under the assumption of suitable compactness, the convergence of the corresponding infima and (almost) minimizers.

By now classical homogenization results via $\Gamma$-convergence include the papers by MARCELlini [46] in the convex setting, as well as the first work in the nonconvex case with standard $p$-growth by MüLler [47] and BRAIDES [7]. Within multiscale analysis, which comprises homogenization and relaxation theory, variational problems with non-convex pointwise or differential constraints are known to be technically challenging, cf. $[9,17,20,30,42]$. Despite recent partial progress towards attacking the issue of localization, i.e. proving that limit functionals preserve integral form, with different methods, e.g. [20,30,40,52], there are still general open questions that cannot be worked out with existing tools. In this article, we investigate homogenization problems subject to a special type of approximate differential inclusion constraint, which do not satisfy standard assumptions and therefore require a tailored approach.

Let $\alpha>0$ and $p \in(1, \infty)$. Consider for each $\varepsilon>0$ the integral functional $E_{\varepsilon}$ defined for $u \in W^{1, p}\left(\Omega ; \mathbb{R}^{n}\right)$ by

$$
E_{\varepsilon}(u)=\int_{\varepsilon Y_{\text {stiff } \cap \Omega}} \frac{1}{\varepsilon^{\alpha}} \operatorname{dist}^{p}(\nabla u, S O(n)) \mathrm{d} x+\int_{\varepsilon Y_{\text {soft } \cap \Omega}} W_{\text {soft }}(\nabla u) \mathrm{d} x
$$

with an integrand $W_{\text {soft }}: \mathbb{R}^{n \times n} \rightarrow \mathbb{R}$, which is in general not convex or quasiconvex. These functionals model the elastic energy of a layered composite. The first term with diverging elastic constants, scaling like $\varepsilon^{-\alpha}$, is the contribution of the stiff components and the second term is associated with the softer components.

In the regime $\alpha>p$, we show that the $\Gamma$-limit of $\left(E_{\varepsilon}\right)_{\varepsilon}$ as $\varepsilon \rightarrow 0$ with respect to strong convergence in $L^{p}\left(\Omega ; \mathbb{R}^{n}\right)$, or equivalently weak convergence in $W^{1, p}\left(\Omega ; \mathbb{R}^{n}\right)$, exists and determine a characterizing formula. The required technical assumptions on the geometry of $\Omega$ are those of Definitions 3.6 and 3.7 and the density $W_{\text {soft }}$ is supposed to satisfy $(H 1)-(H 3)$, see Section 5. In fact, the $\Gamma$-limit has integral form, is subject to the constraints on the admissible macroscopic deformations induced by asymptotic rigidity (cf. Theorem 1.1), and can be expressed purely in terms of the energy density $W_{\text {soft }}$ and the relative thickness $\lambda$ of the softer layers. More precisely,

$$
E_{\mathrm{hom}}(u):=\Gamma-\lim _{\varepsilon \rightarrow 0} E_{\varepsilon}(u)=\int_{\Omega} \lambda W_{\mathrm{soft}}^{\mathrm{qc}}\left(\frac{1}{\lambda}(\nabla u-(1-\lambda) R)\right) \mathrm{d} x
$$

for all $u$ of the form (1.4), and $E_{\mathrm{hom}}(u)=\infty$ otherwise. Here, $W_{\mathrm{soft}}^{\mathrm{qc}}$ stands for the quasiconvex envelope of $W_{\text {soft }}$; for background information on generalized notions of convexity and relaxations, see e.g. [25].

Next, we collect a few remarks to put the above mentioned homogenization result $-\mathrm{a}$ detailed formulation of the full version is given in Theorem 5.2 - in context with related work in the literature.

Remark 1.3. (a) General theorems on homogenization tend to be rather implicit in the sense that they involve (multi)cell formulas (e.g. [7,47]), which again require to solve infinite dimensional minimization problems. In contrast, the 
$\Gamma$-limit in (1.6) is clearly explicit with regards to the macroscopic effect of the heterogeneities. If the relaxation of the softer components, or in other words, the quasiconvexification of $W_{\text {soft }}$, is known, the representation of the homogenized energy density becomes even fully explicit. To illustrate the latter, we discuss the prototypical example of the Saint-Venant Kirchhoff stored energy function in Example 5.1.

(b) As we demonstrate in Remark 5.5, the density in (1.6) coincides with a singlecell formula. This indicates that microstructures ranging over multiple cells (or layers) are not energetically favorable, in contrast with the general theory. Indeed, Müller's well-known counterexample [47], which involves a polyconvex energy density function, gives evidence that multi-cell formulas are necessary in general to describe homogenized limits of non-convex problems (see also [5] for further examples). The recent paper [51] refines this observation by showing that a single-cell formula is sufficient in a neighborhood of rotations, though.

(c) Next, we highlight a selection of related references on the variational analysis of different types of elastic high-contrast composites. The case of stiff inclusions in a softer phase is covered in $[10,30]$, while $[10,13,14]$ study the asymptotics of material models with increasingly soft inclusions. For results on the extreme regime of perforated materials, we refer to the seminal paper [1], and more recently, in the context of brittle elastic materials to [11]. The effective behavior of fiber-reinforced brittle materials is studied in [6].

A common feature of all these results is the isotropy of the derived homogenized energies. In contrast, strong anisotropy at the macroscopic level arises in Theorem 5.2 from the layered geometry of the heterogeneities, especially in the form of restrictions on the class of admissible deformations.

(d) Asymptotic rigidity as a concept and technical tool is not only limited to the homogenization problem in Theorem 5.2. It can be used also in other contexts and has the potential for extensions in different directions, as recent work on the asymptotic analysis of models for layered materials in finite crystal plasticity illustrates, see [16,27] and [15, Chapter 5, 6]. In particular, [27] contains a $B V$ version of Theorem 1.1 in the case of fully rigid components, which makes a first connection with applications in fracture mechanics. A step towards carrying the results to problems in stochastic homogenization is made in [15], by assuming a random distribution of the layer thickness.

We conclude the introduction with a few words about the proof of Theorem 5.2, focussing on the main ideas and technical challenges. The construction of a recovery sequence for affine limit maps (Step 1) is based on laminates made of rotations and shear components (cf. [16, Section 4]), which we augment with suitable perturbations on the softer layers. The harder part is the case of general limits (Step 3). Recall that Theorem 1.1(ii) provides an admissible approximating sequence for any possible limit map as in (1.4). However, these sequences fail to be energetically optimal in general. To remedy this problem, we localize by piecewise constant approximation of the limit functions, which can be done in a constraint preserving way due to the essentially one-dimensional character of the representation in (1.4) (see also (1.5)). Finally, we determine locally optimal microstructures as in the 
affine case and glue them onto the sequence from Theorem 1.1(ii) in the softer parts. This construction is sufficient to recover the energy.

In essence, our reasoning for the liminf-inequality (Steps 2 and 4) comes down to using Theorem 1.1(i) and to applying Jensen's inequality twice, first to obtain a lower bound energy estimate on each softer layer and then, in the optimization process over the entirety of layers. Besides, we employ the properties of NullLagrangians. The presented arguments rely strongly on the hypothesis that $W_{\text {soft }}^{\mathrm{qc}}$ is polyconvex (referred to as $(H 1)$ ), meaning that the quasiconvex envelope can be written as a convex function of the vector of minors, or in other words, that the quasiconvex envelope coincides with the polyconvex one. Notice that the same assumption can be found e.g. in [20] in the context of relaxation problems with constraints on the determinant.

Dropping $(H 1)$ appears to be a non-trivial task. On a technical level, if the Jensen's inequalities mentioned above were to be replaced straight away by the related formulas defining quasiconvexity (see (5.2)), this would require careful cut-off arguments at the boundaries. In the stiff layers, though, cut-off conflicts with the rigidity constraints and difficulties may arise from non-local effects due to interaction between different layers. Hence, it remains an open question to understand whether removing $(H 1)$ from the list of assumptions makes the $\Gamma$-limit $E_{\text {hom }}$ in (1.6) (if existent) smaller. Or in more intuitive terms, can the energy be further reduced by oscillations of the rotation matrices and long range effects over multiple layers?

Structure of the article This paper is organized into five sections. In the subsequent Section 2, we discuss a range of explicit bending examples, which illustrate softer macroscopic behavior in the regimes $0<\alpha \leqq p$ and establish in particular the optimality of the condition $\alpha>p$ in Theorem 1.1(i). Sections 3 and 4 contain the proofs of the asymptotic rigidity result formulated in Theorem 1.1. In Section 3, we prove a generalization of the necessity part (i) as well as Corollary 3.9, followed by a more detailed discussion on the geometric assumptions on the domain $\Omega$. Section 4 proceeds with the proof of the sufficiency statement (ii) of Theorem 1.1. In Section 5, we state our second main result on homogenization via $\Gamma$-convergence, that is Theorem 5.2. For its proof, both parts of Theorem 1.1 are key. We conclude by relating the homogenization formula of (1.6) to the cell formula as it occurs in models of composites with rigid layers. The "Appendix" provides two technical auxiliary results in form of a specialized reverse Poincaré type inequality and a lemma on locally one-dimensional functions.

Notation The standard unit vectors in $\mathbb{R}^{n}$ are denoted by $e_{1}, \ldots, e_{n}$. For the Euclidean inner product between two vectors $a, b \in \mathbb{R}^{n}$ we write $a \cdot b$. Moreover, let $a \otimes b=a b^{T} \in \mathbb{R}^{n \times n}$ for $a, b \in \mathbb{R}^{n}$, and set $a^{\perp}=\left(-a_{n}, a_{2}, \ldots, a_{n-1}, a_{1}\right)^{T} \in \mathbb{R}^{n}$ for $a \in \mathbb{R}^{n}$, which generalizes the usual notation for perpendicular vectors in two dimensions. The Frobenius norm of $A \in \mathbb{R}^{n \times n}$ is given by $|A|=\sqrt{A A^{T}}$. Our notation for block diagonal matrices is $A=\operatorname{diag}\left(A_{1}, A_{2}, \ldots, A_{m}\right) \in \mathbb{R}^{n \times n}$ with $A_{i} \in \mathbb{R}^{n_{i} \times n_{i}}$ and $\sum_{i=1}^{m} n_{i}=n$. In the following, we will often split up $a \in \mathbb{R}^{n}$ as $a=\left(a^{\prime}, a_{n}\right)$, where $a^{\prime}=\left(a_{1}, \ldots, a_{n-1}\right)$. For a matrix $A \in \mathbb{R}^{n \times n}$ a similar splitting into its columns is used, that is $A=\left(A^{\prime} \mid A e_{n}\right)$ with $A^{\prime} \in \mathbb{R}^{n \times(n-1)}$. For $t \in \mathbb{R}$, the 
expressions $\lfloor t\rfloor$ and $\lceil t\rceil$ stand for the largest integer smaller and smallest integer larger than $t$, respectively.

By a domain $\Omega \subset \mathbb{R}^{n}$ we mean an open, connected subset of $\mathbb{R}^{n}$. An open cuboid is the Cartesian product $Q=\left(a_{1}, b_{1}\right) \times \cdots \times\left(a_{n}, b_{n}\right)=: \times_{i}\left(a_{i}, b_{i}\right) \subset \mathbb{R}^{n}$ with $a_{i}, b_{i} \in \mathbb{R}$ and $a_{i}<b_{i}$ for $i=1, \ldots, n$. Hence for us, cuboids will always be oriented along the coordinate axes. Furthermore, $\mathbb{1}_{E}$ and $\chi_{E}$ are the indicator and characteristic function corresponding to a subset $E \subset \mathbb{R}^{n}$, i.e., $\mathbb{1}_{E}(x)=1$ and $\chi_{E}(x)=0$ if $x \in E$, and $\mathbb{1}_{E}(x)=0$ and $\chi_{E}(x)=\infty$ if $x \notin E$. For a measurable set $U$ and an integrable function $f: U \rightarrow \mathbb{R}^{m}$, let $f_{U} f \mathrm{~d} x:=\frac{1}{|U|} \int_{U} f \mathrm{~d} x$.

We use the common notation for Lebesgue and Sobolev spaces, as well as for function spaces of continuously differentiable functions. By $L_{0}^{p}\left(\Omega ; \mathbb{R}^{m}\right)$, we denote the space of functions in $L^{p}\left(\Omega ; \mathbb{R}^{m}\right)$ with the property that their mean value vanishes. Periodic boundary condition are indicated by a lower case \#, for example in $W_{\#}^{1, p}\left(Y ; \mathbb{R}^{m}\right)$.

The distributional derivative of a function $f \in L_{\text {loc }}^{1}\left(\Omega ; \mathbb{R}^{m}\right)$ is denoted by $D f$, for partial derivatives in the $e_{i}$-direction we write $\partial_{i} u$. Moreover, $D f=\left(D^{\prime} f \mid \partial_{n} f\right)$ with $D^{\prime} f=\left(\partial_{1} f|\ldots| \partial_{n-1} f\right)$. If $f: \Omega \rightarrow \mathbb{R}^{m}$ is classically or weakly differentiable, we denote the (weak) gradient of $f$ by $\nabla f$. Here again, one has the splitting $\nabla f=\left(\nabla^{\prime} f \mid \partial_{n} f\right)$ with $\nabla^{\prime} f=\left(\partial_{1} f|\ldots| \partial_{n-1} f\right)$. In case $f: J \rightarrow \mathbb{R}^{m}$ is a onedimensional function with $J \subset \mathbb{R}$ an open interval, we simply write $f^{\prime}$ for the derivative of $f$.

Convergence of a sequence $\left(u_{\varepsilon}\right)_{\varepsilon}$ as $\varepsilon \rightarrow 0$ means that $\left(u_{\varepsilon_{j}}\right)_{j}$ converges as $j \rightarrow \infty$ for any subsequence $\varepsilon_{j} \downarrow 0$. Note finally the use of generic constants, mostly denoted by $c$ or $C$, which may vary from line to line without change in notation.

\section{Optimality of the Scaling Regimes}

While for $\alpha=0$ in (3.2) the class of effective deformations with finite energy comprises arbitrary Sobolev maps with vanishing mean value, the material response in the case " $\alpha=\infty$ " is rather rigid. This raises the natural question up to which value of $\alpha$ softer material response can be encountered. In this section, we discuss four examples of macroscopically attainable deformations. They show that Theorem 1.1 and Corollary 3.9 fail for small elastic constants in the regime $\alpha \leqq p$, and illustrate the effect of (local) volume preservation. For simplicity, we assume throughout this section that $\Omega \subset \mathbb{R}^{n}$ is the unit cube, i.e. $\Omega=(0,1)^{n}$.

The idea behind the first two constructions for $\alpha=p$ is to bend the individual stiffer layers, first uniformly in Example 2.3, and then in a locally volumepreserving way inspired by the bending of a stack of paper in Example 2.4. Example 2.5 is based on a wrinkling construction for the individual layers, and shows that compression in layer direction is possible for $\alpha \in(0, p)$. Finally, we look into the effect of the local volume condition for $\alpha>p$ in Example 2.6.

The calculations behind these examples share a common structure and are all based on the following auxiliary result. We deliberately keep its formulation slightly more general than actually needed in what follows. This facilitates the construction 
of an even larger variety of explicit deformations and yields immediate insight into their asymptotic properties.

As regards notation, we write $\llbracket x \rrbracket_{\varepsilon}$ for the orthogonal projection of $x \in \varepsilon Y_{\text {stiff }}$ onto the midsection of the stiff layer containing $x$; if $x \in \varepsilon Y_{\text {soft }}, \llbracket x \rrbracket_{\varepsilon}$ refers to the projection onto the midsection of the closest stiff layer above $x$. Precisely, for $\varepsilon>0$ and $t \in \mathbb{R}$, we let $[t]_{\varepsilon}=\varepsilon\left\lceil\frac{t}{\varepsilon}\right\rceil-\varepsilon+\frac{1+\lambda}{2} \varepsilon$, so that

$$
\llbracket x \rrbracket_{\varepsilon}=\left(x_{1}, \ldots, x_{n-1},\left[x_{n}\right]_{\varepsilon}\right) \text { for } x \in \mathbb{R}^{n} .
$$

Due to

$$
\left|x_{n}-\left[x_{n}\right]_{\varepsilon}\right|=\varepsilon\left|\frac{x_{n}}{\varepsilon}-\left\lceil\frac{x_{n}}{\varepsilon}\right\rceil+1-\frac{1+\lambda}{2}\right| \leqq 2 \varepsilon \text { for any } x \in \mathbb{R}^{n} \text {, }
$$

we observe that $\llbracket x \rrbracket_{\varepsilon} \rightarrow x$ as $\varepsilon \rightarrow 0$.

Lemma 2.1. Let $\bar{Q}=[0,1]^{n-1} \times\left[-\frac{1}{2}, 2\right]$ and $1<p<\infty$. For $\varepsilon \in(0,1)$, let $f_{\varepsilon} \in C^{2}\left(\bar{Q} ; \mathbb{R}^{n}\right)$ be such that $\left|\partial_{1} f_{\varepsilon}\right|=1, \partial_{1} f_{\varepsilon} \in \operatorname{span}\left\{e_{1}, e_{n}\right\}$ and $\partial_{i} f_{\varepsilon}=e_{i}$ for $i=2, \ldots, n-1$, and define a Lipschitz function $u_{\varepsilon}: \Omega \rightarrow \mathbb{R}^{n}$ by

$$
u_{\varepsilon}(x)=f_{\varepsilon}\left(\llbracket x \rrbracket_{\varepsilon}\right)+\left(x_{n}-\left[x_{n}\right]_{\varepsilon}\right) \partial_{1} f_{\varepsilon}^{\perp}\left(\llbracket x \rrbracket_{\varepsilon}\right) \text { for } x \in \varepsilon Y_{\text {stiff }} \cap \Omega,
$$

and by linear interpolation in the $e_{n}$-direction in $\varepsilon Y_{\mathrm{soft}} \cap \Omega$.

Then, for any $\varepsilon \in(0,1)$,

$$
\int_{\varepsilon Y_{\text {stiff }} \cap \Omega} \operatorname{dist}^{p}\left(\nabla u_{\varepsilon}, S O(n)\right) \mathrm{d} x \leqq 2^{p} \varepsilon^{p}\left\|\partial_{11}^{2} f_{\varepsilon}\right\|_{L^{\infty}\left(\bar{Q} ; \mathbb{R}^{n}\right)}^{p} .
$$

Moreover, if $\lim _{\mathcal{\varepsilon} \rightarrow 0} \varepsilon\left\|\nabla^{2} f_{\varepsilon}\right\|_{L^{\infty}\left(\bar{Q} ; \mathbb{R}^{n \times n \times n}\right)}=0$ and if there is $F \in L^{p}\left(\bar{Q} ; \mathbb{R}^{n \times n}\right)$ such that either

(i) $\nabla f_{\varepsilon} \rightarrow F$ in $L^{p}\left(\bar{Q} ; \mathbb{R}^{n \times n}\right)$ as $\varepsilon \rightarrow 0$, or

(ii) $\nabla f_{\varepsilon} \rightarrow F$ in $L^{p}\left(\bar{Q} ; \mathbb{R}^{n \times n}\right)$ as $\varepsilon \rightarrow 0$ and $\partial_{n}\left(\nabla f_{\varepsilon}\right)=0$ for all $\varepsilon \in(0,1)$,

then

$$
\nabla u_{\varepsilon} \rightarrow F \text { in } L^{p}\left(\Omega ; \mathbb{R}^{n \times n}\right) .
$$

Remark 2.2. The choice of $\bar{Q}$ as the domain of the functions $f_{\varepsilon}$ ensures that $u_{\varepsilon}$ as in (2.2) is well-defined. Indeed, if $t \in[0,1]$ and $\varepsilon \in(0,1)$, then $-\frac{1}{2}<-\frac{\varepsilon}{2} \leqq$ $[t]_{\varepsilon} \leqq 1+\varepsilon<2$.

Proof. By definition, the functions $u_{\varepsilon}$ are continuously differentiable on the connected components of $\varepsilon Y_{\text {stiff }} \cap \Omega$ and $\varepsilon Y_{\text {soft }} \cap \Omega$. Then,

$$
\begin{aligned}
\nabla u_{\varepsilon}(x)= & \partial_{1} f_{\varepsilon}\left(\llbracket x \rrbracket_{\varepsilon}\right) \otimes e_{1}+\left(x_{n}-\left[x_{n}\right]_{\varepsilon}\right) \partial_{11}^{2} f_{\varepsilon}^{\perp}\left(\llbracket x \rrbracket_{\varepsilon}\right) \otimes e_{1} \\
& +\sum_{i=2}^{n-1} e_{i} \otimes e_{i}+\partial_{1} f_{\varepsilon}^{\perp}\left(\llbracket x \rrbracket_{\varepsilon}\right) \otimes e_{n}
\end{aligned}
$$


for $x \in \varepsilon Y_{\text {stiff }} \cap \Omega$, and a straight-forward calculation yields the gradients for $x \in \varepsilon Y_{\text {soft }} \cap \Omega$,

$$
\begin{aligned}
\nabla u_{\varepsilon}(x)= & \left(\partial_{1} f_{\varepsilon}\left(\llbracket x \rrbracket_{\varepsilon}-\varepsilon e_{n}\right)+\frac{1-\lambda}{2} \varepsilon \partial_{11}^{2} f_{\varepsilon}^{\perp}\left(\llbracket x \rrbracket_{\varepsilon}-\varepsilon e_{n}\right)\right) \otimes e_{1} \\
& +\frac{1}{\lambda \varepsilon}\left(x_{n}-\left\lceil\frac{x_{n}}{\varepsilon} \rrbracket \varepsilon+\varepsilon\right)\left(\partial_{1} f_{\varepsilon}\left(\llbracket x \rrbracket_{\varepsilon}\right)-\partial_{1} f_{\varepsilon}\left(\llbracket x \rrbracket_{\varepsilon}-\varepsilon e_{n}\right)\right) \otimes e_{1}\right. \\
& -\frac{1-\lambda}{2 \lambda}\left(x_{n}-\left\lceil\frac{x_{n}}{\varepsilon}\right\rceil \varepsilon+\varepsilon\right)\left(\partial_{11}^{2} f_{\varepsilon}^{\perp}\left(\llbracket x \rrbracket_{\varepsilon}\right)+\partial_{11}^{2} f_{\varepsilon}^{\perp}\left(\llbracket x \rrbracket_{\varepsilon}-\varepsilon e_{n}\right)\right) \otimes e_{1} \\
& +\sum_{i=2}^{n-1} e_{i} \otimes e_{i}+\frac{1}{\lambda \varepsilon}\left(f_{\varepsilon}\left(\llbracket x \rrbracket_{\varepsilon}\right)-f_{\varepsilon}\left(\llbracket x \rrbracket_{\varepsilon}-\varepsilon e_{n}\right)\right) \otimes e_{n} \\
& -\frac{1-\lambda}{2 \lambda}\left(\partial_{1} f_{\varepsilon}^{\perp}\left(\llbracket x \rrbracket_{\varepsilon}\right)+\partial_{1} f_{\varepsilon}^{\perp}\left(\llbracket x \rrbracket_{\varepsilon}-\varepsilon e_{n}\right)\right) \otimes e_{n},
\end{aligned}
$$

see [15, Lemma 3.4.3]) for more details.

In view of (2.5) and the observation that $\partial_{1} f_{\varepsilon}\left(\llbracket x \rrbracket_{\varepsilon}\right) \otimes e_{1}+\sum_{i=2}^{n-1} e_{i} \otimes e_{i}+$ $\partial_{1} f_{\varepsilon}^{\perp}\left(\llbracket x \rrbracket_{\varepsilon}\right) \otimes e_{n} \in S O(n)$ for all $x \in \Omega$ due to $\left|\partial_{1} f_{\varepsilon}\right|=\left|\partial_{1} f_{\varepsilon}^{\perp}\right|=1$ and $\partial_{1} f_{\varepsilon} \in \operatorname{span}\left\{e_{1}, e_{n}\right\}$, the elastic energy contribution on the stiffer layers can be estimated by

$$
\begin{aligned}
\int_{\varepsilon Y_{\text {stiff }} \cap \Omega} \operatorname{dist}^{p}\left(\nabla u_{\varepsilon}, S O(n)\right) \mathrm{d} x & \leqq \int_{\varepsilon Y_{\text {stiff } \cap \Omega}}\left|\left(x_{n}-\left[x_{n}\right]_{\varepsilon}\right) \partial_{11}^{2} f_{\varepsilon}^{\perp}\left(\llbracket x \rrbracket_{\varepsilon}\right) \otimes e_{1}\right|^{p} \mathrm{~d} x \\
& \leqq\left\|\partial_{11}^{2} f_{\varepsilon}\right\|_{L^{\infty}\left(\bar{Q} ; \mathbb{R}^{n}\right)}^{p} \int_{\Omega}\left|x_{n}-\left[x_{n}\right]_{\varepsilon}\right|^{p} \mathrm{~d} x
\end{aligned}
$$

By (2.1), this implies (2.3).

For the proof of (2.4), consider the auxiliary fields $V_{\varepsilon} \in L^{\infty}\left(\Omega ; \mathbb{R}^{n \times n}\right)$ given by

$$
\begin{aligned}
V_{\varepsilon}= & \partial_{1} f_{\varepsilon} \otimes e_{1}+\sum_{i=2}^{n-1} e_{i} \otimes e_{i}+\left(\partial_{1} f_{\varepsilon}^{\perp} \otimes e_{n}\right) \mathbb{1}_{\varepsilon} Y_{\text {stiff }} \cap \Omega \\
& +\left(\left(\frac{1}{\lambda} \partial_{n} f_{\varepsilon}-\frac{1-\lambda}{\lambda} \partial_{1} f_{\varepsilon}^{\perp}\right) \otimes e_{n}\right) \mathbb{1}_{\varepsilon} Y_{\text {soft }} \cap \Omega .
\end{aligned}
$$

Recall that the indicator function associated with a set $E \subset \mathbb{R}^{n}$ is denoted by $\mathbb{1}_{E}$. We will show that

$$
V_{\varepsilon}-\nabla u_{\varepsilon} \rightarrow 0 \text { in } L^{\infty}\left(\Omega ; \mathbb{R}^{n \times n}\right)
$$

Indeed, along with the mean value theorem and (2.1), one obtains for $x$ in the interior of $\varepsilon Y_{\text {stiff }} \cap \Omega$ that

$$
\begin{aligned}
\mid \nabla & u_{\varepsilon}(x)-V_{\varepsilon}(x) \mid \\
\leqq & \left|\left(\partial_{1} f_{\varepsilon}\left(\llbracket x \rrbracket_{\varepsilon}\right)-\partial_{1} f_{\varepsilon}(x)\right) \otimes e_{1}\right|+\left|\left(x_{n}-\left[x_{n}\right]_{\varepsilon}\right) \partial_{11}^{2} f_{\varepsilon}^{\perp}\left(\llbracket x \rrbracket_{\varepsilon}\right) \otimes e_{n}\right| \\
& +\left|\left(\partial_{1} f_{\varepsilon}^{\perp}\left(\llbracket x \rrbracket_{\varepsilon}\right)-\partial_{1} f_{\varepsilon}^{\perp}(x)\right) \otimes e_{n}\right| \\
\leqq & \left|x_{n}-\left[x_{n}\right]_{\varepsilon}\right|\left(\left\|\partial_{1 n}^{2} f_{\varepsilon}\right\|_{L^{\infty}\left(\bar{Q} ; \mathbb{R}^{n}\right)}+\left\|\partial_{11}^{2} f_{\varepsilon}\right\|_{L^{\infty}\left(\bar{Q} ; \mathbb{R}^{n}\right)}+\left\|\partial_{1 n}^{2} f_{\varepsilon}^{\perp}\right\|_{L^{\infty}\left(\bar{Q} ; \mathbb{R}^{n}\right)}\right) \\
\leqq & 6 \varepsilon\left\|\nabla^{2} f_{\varepsilon}\right\|_{L^{\infty}\left(\bar{Q} ; \mathbb{R}^{n \times n \times n}\right)},
\end{aligned}
$$


and, similarly, for $x \in \varepsilon Y_{\text {soft }} \cap \Omega$,

$$
\begin{aligned}
\left|\nabla u_{\varepsilon}(x)-V_{\varepsilon}(x)\right| \leqq & \left|\nabla u_{\varepsilon} e_{1}(x)-V_{\varepsilon} e_{1}(x)\right|+\left|\nabla u_{\varepsilon} e_{n}(x)-V_{\varepsilon} e_{n}(x)\right| \\
\leqq & 3 \varepsilon\left(\frac{1+\lambda}{\lambda}\right)\left\|\partial_{1 n}^{2} f_{\varepsilon}\right\|_{L^{\infty}\left(\bar{Q} ; \mathbb{R}^{n}\right)}+\frac{2}{\lambda} \varepsilon\left\|\partial_{11}^{2} f_{\varepsilon}^{\perp}\right\|_{L^{\infty}\left(\bar{Q} ; \mathbb{R}^{n}\right)} \\
& +\frac{3}{\lambda} \varepsilon\left\|\partial_{1 n}^{2} f_{\varepsilon}\right\|_{L^{\infty}\left(\bar{Q} ; \mathbb{R}^{n}\right)}+\frac{1}{\lambda}\left|\partial_{n} f_{\varepsilon}\left(x_{1}, \xi\right)-\partial_{n} f_{\varepsilon}(x)\right| \\
\leqq & 6 \varepsilon\left(\frac{1+\lambda}{\lambda}\right)\left(\left\|\partial_{1 n}^{2} f_{\varepsilon}\right\|_{L^{\infty}\left(\bar{Q} ; \mathbb{R}^{n}\right)}+\left\|\partial_{11}^{2} f_{\varepsilon}\right\|_{L^{\infty}\left(\bar{Q} ; \mathbb{R}^{n}\right)}\right. \\
& \left.+\left\|\partial_{n n}^{2} f_{\varepsilon}\right\|_{L^{\infty}\left(\bar{Q} ; \mathbb{R}^{n}\right)}\right) \\
\leqq & 18 \varepsilon\left(\frac{1+\lambda}{\lambda}\right)\left\|\nabla^{2} f_{\varepsilon}\right\|_{L^{\infty}\left(\bar{Q} ; \mathbb{R}^{n \times n \times n}\right)},
\end{aligned}
$$

with some $\xi \in\left(\left[x_{n}\right]_{\varepsilon}-\varepsilon,\left[x_{n}\right]_{\varepsilon}\right)$. Accounting for $\lim _{\varepsilon \rightarrow 0} \varepsilon\left\|\nabla^{2} f_{\varepsilon}\right\|_{L^{\infty}\left(\bar{Q} ; \mathbb{R}^{n \times n \times n}\right)}=$ 0 leads to (2.7).

In case (i), it follows from (2.6) along with a weak-strong convergence argument that

$$
\begin{aligned}
& V_{\varepsilon} \rightarrow F e_{1} \otimes e_{1}+\sum_{i=2}^{n-1} e_{i} \otimes e_{i}+(1-\lambda)\left(F e_{1}\right)^{\perp} \otimes e_{n} \\
& \quad+F e_{n} \otimes e_{n}-(1-\lambda)\left(F e_{1}\right)^{\perp} \otimes e_{n}=F \quad \text { in } L^{1}\left(\Omega ; \mathbb{R}^{n \times n}\right),
\end{aligned}
$$

where we have used in particular that $\mathbb{1}_{\varepsilon} Y_{\text {stiff } \cap \Omega} \stackrel{*}{\rightarrow}(1-\lambda)$ and $\mathbb{1}_{\varepsilon} Y_{\text {soft }} \cap \Omega \stackrel{*}{\rightarrow} \lambda$ in $L^{\infty}(\Omega)$, as well as $F e_{i}=e_{i}$ for $i=2, \ldots, n-1$. The latter follows directly from the assumption that $\partial_{i} f_{\varepsilon}=e_{i}$ for all $\varepsilon \in(0,1)$ if $i=2, \ldots, n-1$.

Combining (2.8) and (2.7) shows that $\nabla u_{\varepsilon} \rightarrow F$ in $L^{1}\left(\Omega ; \mathbb{R}^{n \times n}\right)$. Since $\left(\nabla u_{\varepsilon}\right)_{\varepsilon}$ is uniformly bounded in $L^{p}\left(\Omega ; \mathbb{R}^{n \times n}\right)$ by (2.3) and the requirement that $\varepsilon\left\|\partial_{11}^{2} f_{\varepsilon}\right\|_{L^{\infty}\left(\bar{Q} ; \mathbb{R}^{n}\right)} \rightarrow 0$, we finally infer (2.4), which finishes the proof under the assumption of (i).

If assumption (ii) is satisfied, then $\partial_{1} f_{\varepsilon}^{\perp}$ depends only on $x_{1}$. Since $\mathbb{1}_{\varepsilon} Y_{\text {stiff }}$ on the other hand is constant in the $x_{1}$-variable, we observe a separation of variables in the product $\left(\partial_{1} f_{\varepsilon}^{\perp}\right) \mathbb{1}_{\varepsilon} Y_{\text {stiff }}$. In light of this observation, consider test functions $\varphi \in C^{0}\left(\bar{\Omega} ; \mathbb{R}^{n}\right)$ of the form $\varphi(x)=(\phi \otimes \psi)(x):=\phi\left(x_{1}\right) \psi\left(x_{2}, \ldots, x_{n}\right)$ for $x \in \bar{\Omega}$ with $\phi \in C^{0}\left([0,1] ; \mathbb{R}^{n}\right)$ and $\psi \in C^{0}\left([0,1]^{n-1}\right)$. Then, due to Fubini's theorem and the lemma on weak convergence of rapidly oscillating periodic functions (see e.g. [18, Section 2.3]), it follows that

$$
\begin{aligned}
& \int_{\Omega}\left(\partial_{1} f_{\varepsilon}^{\perp} \cdot \varphi\right) \mathbb{1}_{\varepsilon} Y_{\text {stiff }} \cap \Omega \mathrm{d} x \\
& \quad=\left(\int_{[0,1]} \partial_{1} f_{\varepsilon}^{\perp} \cdot \phi \mathrm{d} x_{1}\right)\left(\int_{[0,1]^{n-1}} \mathbb{1}_{\varepsilon Y_{\text {stiff }} \cap \Omega} \psi \mathrm{d} x_{2} \ldots \mathrm{d} x_{n}\right) \\
& \quad \rightarrow\left(\int_{[0,1]}\left(F e_{1}\right)^{\perp} \phi\left(x_{1}\right) \mathrm{d} x_{1}\right)\left(\int_{[0,1]^{n-1}}(1-\lambda) \psi \mathrm{d} x_{2} \ldots \mathrm{d} x_{n}\right) \\
& =(1-\lambda) \int_{\Omega}\left(F e_{1}\right)^{\perp} \cdot \varphi \mathrm{d} x \text { as } \varepsilon \rightarrow 0 .
\end{aligned}
$$

We recall that as a corollary of the Stone-Weierstrass theorem (see e.g. [54, Theorem 7.32]) and the density of $C^{0}\left(\bar{\Omega} ; \mathbb{R}^{n}\right)$ in $L^{q}\left(\Omega ; \mathbb{R}^{n}\right)$ with $1 \leqq q<\infty$, the span of functions $\phi \otimes \psi$ is dense in $L^{q}\left(\Omega ; \mathbb{R}^{n}\right)$. Consequently, we infer from (2.9) that

$$
\left(\partial_{1} f_{\varepsilon}^{\perp}\right) \mathbb{1}_{\varepsilon} Y_{\text {stiff }} \cap \Omega \rightarrow(1-\lambda)\left(F e_{1}\right)^{\perp} \text { in } L^{p}\left(\Omega ; \mathbb{R}^{n}\right) .
$$




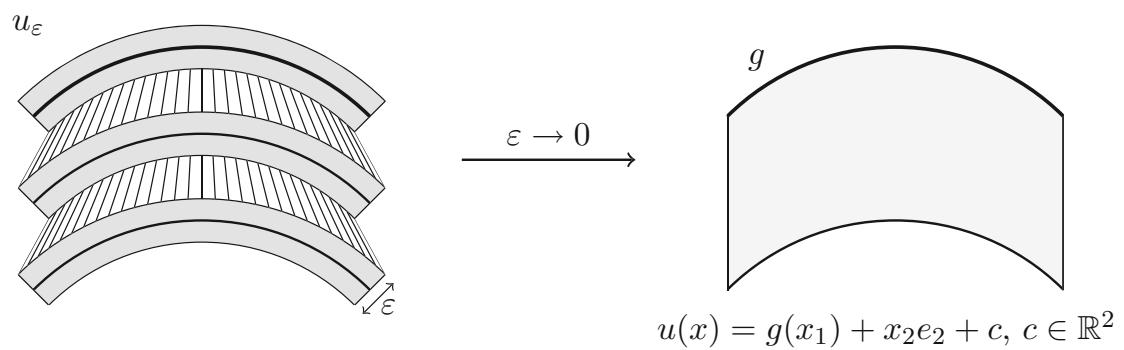

Fig. 2. Illustration of the deformations of Example 2.3 for $n=2$, with uniform bending of the stiffer layers described by $g(t)=\sin \left(t-\frac{1}{2}\right) e_{1}+\cos \left(t-\frac{1}{2}\right) e_{2}$ for $t \in[0,1]$

Then the third term in (2.6) converges weakly to $(1-\lambda)\left(F e_{1}\right)^{\perp} \otimes e_{n}$ in $L^{p}\left(\Omega ; \mathbb{R}^{n \times n}\right)$. Arguing similarly for the other product terms in (2.6) eventually yields $V_{\varepsilon} \rightarrow F$ in $L^{p}\left(\Omega ; \mathbb{R}^{n \times n}\right)$. In conjunction with (2.7) this proves (2.4), and thus the statement in case (ii).

As announced at the beginning of the section, we will next discuss four specializations of Lemma 2.1, using the same notations. These examples illustrate the optimality of the scaling regimes in Theorem 1.1 and Corollary 3.9.

Example 2.3. (Uniform bending of the individual stiffer layers) Let $g:[0,1] \rightarrow$ $\operatorname{span}\left\{e_{1}, e_{n}\right\} \subset \mathbb{R}^{n}$ be a $C^{2}$-curve parametrized by arc length, i.e., $\left|g^{\prime}(t)\right|=1$ for all $t \in[0,1]$. We follow Lemma 2.1 to define deformations $u_{\varepsilon}$ by choosing for all $\varepsilon \in(0,1)$,

$$
f_{\varepsilon}(y)=f(y):=g\left(y_{1}\right)+\sum_{i=2}^{n} y_{i} e_{i}, \quad y \in \bar{Q} .
$$

This choice of $f$ is motivated by uniform bending of the individual stiffer layers in the two-dimensional setting, where the curve $g$ describes the bending of the mid-fibers, see Fig. 2.

Then, Lemma 2.1 implies that for any constant $C>2^{p}\left\|g^{\prime \prime}\right\|_{L^{\infty}\left(0,1 ; \mathbb{R}^{n}\right)}^{p}$,

$$
\int_{\varepsilon Y_{\text {stiff }} \cap \Omega} \operatorname{dist}^{p}\left(\nabla u_{\varepsilon}, S O(n)\right) \mathrm{d} x \leqq C \varepsilon^{p},
$$

which shows that the sequence $\left(u_{\varepsilon}\right)_{\varepsilon}$ has finite elastic energy on the stiffer component for $\alpha=p$. As for the gradient of the limit deformation $u$, we infer from version i) of Lemma 2.1 that $\nabla u_{\varepsilon} \rightarrow \nabla u=\nabla f$ in $L^{p}\left(\Omega ; \mathbb{R}^{n \times n}\right)$. In view of (2.10),

$$
\nabla u(x)=g^{\prime}\left(x_{1}\right) \otimes e_{1}+\sum_{i=2}^{n} e_{i} \otimes e_{i}=R(x)+\tilde{a}(x) \otimes e_{n}, \quad x \in \Omega,
$$

with $R(x)=g^{\prime}\left(x_{1}\right) \otimes e_{1}+\sum_{i=1}^{n-1} e_{i} \otimes e_{i}+g^{\prime}\left(x_{1}\right)^{\perp} \otimes e_{n}$ and $\tilde{a}(x)=e_{n}-g^{\prime}\left(x_{1}\right)^{\perp}$. Clearly, for general $g, \partial_{1} R \neq 0$, so that the limit deformation $u$ does not have the form (3.3) obtained in Theorem 1.1 for the regime $\alpha>p$. 
We remark that the limit deformation $u$ is not locally volume preserving for $g$ with non-trivial curvature, since det $\nabla u=g^{\prime} \cdot e_{1} \not \equiv 1$.

To recover limit deformations that satisfy the local volume constraint, a slightly more involved bending construction as in the next example is needed.

Example 2.4. (Macroscopically volume-preserving bending deformations) In the context of Lemma 2.1, we consider for $\varepsilon \in(0,1)$ the functions

$$
f_{\varepsilon}(y)=f(y):=\left(y_{n}+1\right) g\left(\frac{y_{1}}{y_{n}+1}\right)+\sum_{i=2}^{n-1} y_{i} e_{i}, \quad y \in \bar{Q},
$$

with $g:[0,2] \rightarrow \operatorname{span}\left\{e_{1}, e_{n}\right\} \subset \mathbb{R}^{n}$ a $C^{2}$-curve parametrized by arclength.

Then the sequence $\left(u_{\varepsilon}\right)_{\varepsilon}$ defined by (2.2) in the stiffer component and by linear interpolation in the softer one satisfies

$$
\int_{\varepsilon Y_{\text {stiff } \cap \Omega}} \operatorname{dist}^{p}\left(\nabla u_{\varepsilon}, S O(n)\right) \mathrm{d} x \leqq 2^{p}\left\|g^{\prime \prime}\right\|_{L^{\infty}\left(0,2 ; \mathbb{R}^{n}\right)}^{p} \varepsilon^{p},
$$

and we obtain that $\nabla u_{\varepsilon} \rightarrow \nabla u=\nabla f$ in $L^{p}\left(\Omega ; \mathbb{R}^{n \times n}\right)$. Due to

$$
\begin{aligned}
\nabla f(x)= & g^{\prime}\left(\frac{x_{1}}{x_{n}+1}\right) \otimes e_{1}+\sum_{i=2}^{n-1} e_{i} \otimes e_{i} \\
& -\frac{x_{1}}{x_{n}+1} g^{\prime}\left(\frac{x_{1}}{x_{n}+1}\right) \otimes e_{n}+g\left(\frac{x_{1}}{x_{n}+1}\right) \otimes e_{n}
\end{aligned}
$$

for $x \in \Omega$, one can rewrite the gradient of the limit deformation $u$ with the help of a map of rotations $R \in L^{\infty}(\Omega ; S O(n))$ defined for $x \in \Omega$ by $R(x) e_{1}=g^{\prime}\left(\frac{x_{1}}{x_{n}+1}\right)$ and $R(x) e_{i}=e_{i}$ for $i=2, \ldots, n-1$. Precisely,

$$
\nabla u=R+\tilde{a} \otimes e_{n},
$$

with $\tilde{a}(x)=-\frac{x_{1}}{x_{n}+1} g^{\prime}\left(\frac{x_{1}}{x_{n}+1}\right)+g\left(\frac{x_{1}}{x_{n}+1}\right)-g^{\prime}\left(\frac{x_{1}}{x_{n}+1}\right)^{\perp}$ for $x \in \Omega$. The rotations $R$ depend non-trivially on $x_{1}$, hence, the limit map $u$ is not in compliance with Theorem 1.1. Since det $\nabla u=\operatorname{det} \nabla f=-g^{\prime} \cdot g^{\perp}$, the deformation $u$ is locally volume preserving if we chose $g$ such that $g^{\prime} \cdot g^{\perp} \equiv 1$.

An simple deformation of this type, which is intuitively inspired by the bending of a stack of paper, is depicted in Fig. 3.

Next, we discuss an example in the regime $\alpha<p$, where macroscopic shortening in the $e_{1}$-direction occurs due to wrinkling of the stiffer layers. A similar effect occurs in the context of plate theory, cf. [33, Section 5].

Example 2.5. (Wrinkling of stiffer layers) Let $\beta \in \mathbb{R}, \gamma \in(0,1)$, and $g:[0,1] \rightarrow$ $\operatorname{span}\left\{e_{1}, e_{n}\right\} \subset \mathbb{R}^{n}$ be a 1 -periodic $C^{2}$-function with $\left|g^{\prime}(t)\right|=1$ for all $t \in \mathbb{R}$. We define $g_{\varepsilon}:[0,1] \rightarrow \mathbb{R}^{n}$ by $g_{\varepsilon}(t)=\varepsilon^{\gamma} g\left(\varepsilon^{-\gamma} t\right)$ for $t \in[0,1]$ and $\varepsilon \in(0,1)$, and observe that by the weak convergence of periodically oscillating sequences,

$$
g_{\varepsilon}^{\prime} \rightarrow \bar{g}^{\prime}:=\int_{0}^{1} g^{\prime}(t) \mathrm{d} t=g(1)-g(0) \text { in } L^{1}\left(0,1 ; \mathbb{R}^{n}\right) .
$$


$u_{\varepsilon}$

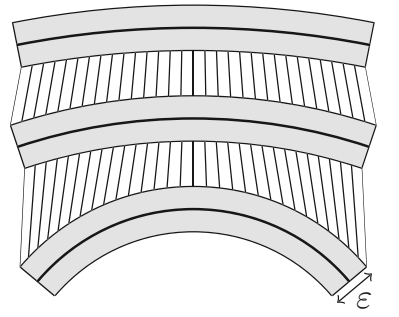

$u$
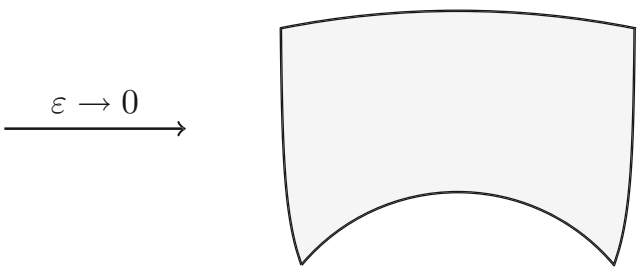

Fig. 3. Illustration of the deformations of Example 2.4 for $n=2$, with $g(t)=\sin (t-$ $\left.\frac{1}{2}\right) e_{1}+\cos \left(t-\frac{1}{2}\right) e_{2}$ for $t \in[0,2]$. Notice also that the limit deformation $u$ satisfies the local volume constraint, whereas the bending deformations of the individual layers in the left picture do not
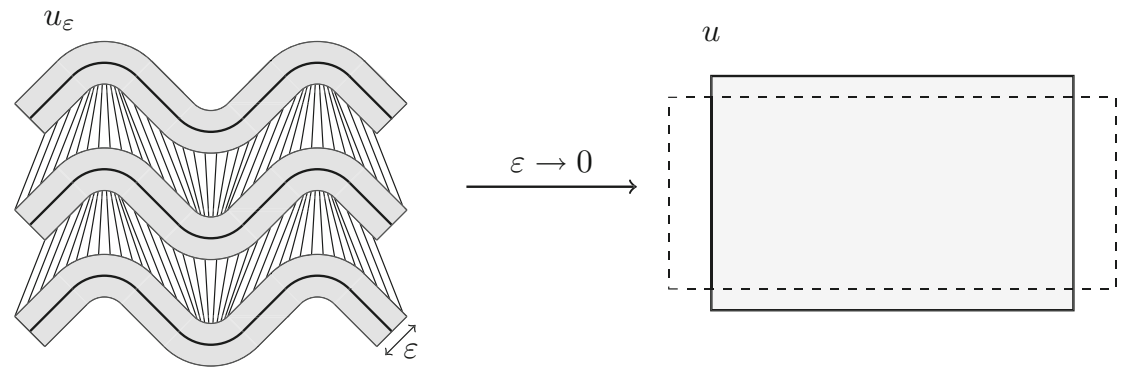

Fig. 4. Illustration of the deformation in Example 2.5 for $n=2$

Unless $g^{\prime}$ is constant, $\left|\bar{g}^{\prime}\right|<1$. Under these assumptions, the functions

$$
f_{\varepsilon}(y)=g_{\varepsilon}\left(y_{1}\right)+\beta y_{n} e_{n}+\sum_{i=2}^{n-1} y_{i} e_{i}, \quad y \in \bar{Q}
$$

meet the requirements of Lemma 2.1 with assumption (ii) and $F=\bar{g}^{\prime} \otimes e_{1}+$ $\sum_{i=2}^{n-1} e_{i} \otimes e_{i}+\beta e_{n} \otimes e_{n}$. Thus, for $u_{\varepsilon}$ as in Lemma 2.1,

$$
\begin{aligned}
& \int_{\varepsilon Y_{\text {stiff }} \cap \Omega} \operatorname{dist}^{p}\left(\nabla u_{\varepsilon}, S O(n)\right) \mathrm{d} x \leqq 2^{p} \varepsilon^{p}\left\|g_{\varepsilon}^{\prime \prime}\right\|_{L^{\infty}\left(0,1 ; \mathbb{R}^{n}\right)}^{p} \\
& \quad \leqq 2^{p} \varepsilon^{p(1-\gamma)}\left\|g^{\prime \prime}\right\|_{L^{\infty}\left(0,1 ; \mathbb{R}^{n}\right)}^{p} \leqq C \varepsilon^{p(1-\gamma)}
\end{aligned}
$$

and $\nabla u_{\varepsilon} \rightarrow \nabla u=F$ in $L^{1}\left(\Omega ; \mathbb{R}^{n \times n}\right)$. In particular, $\left|(\nabla u) e_{1}\right|=\left|F e_{1}\right|=\left|\bar{g}^{\prime}\right|<1$.

Since det $\nabla u=\operatorname{det} F=\beta\left(\bar{g}^{\prime} \cdot e_{1}\right)$, (local) volume preservation of the limit deformation $u$ can be achieved by a suitable choice of $\beta$ and $g$. Graphically speaking, $\beta$ can be viewed as a stretching factor in the $e_{n}$-direction that compensates the loss of length in the $e_{1}$-direction due to the asymptotic shortening of the mid-fibers in the stiffer layers, so that overall volume is preserved. A specific case of this wrinkling construction is depicted in Fig. 4.

Our last example highlights the role of the local volume constraint of the limit deformation in the regime $\alpha \geqq 0$. In particular, it shows that for $\alpha>p$ local volume 

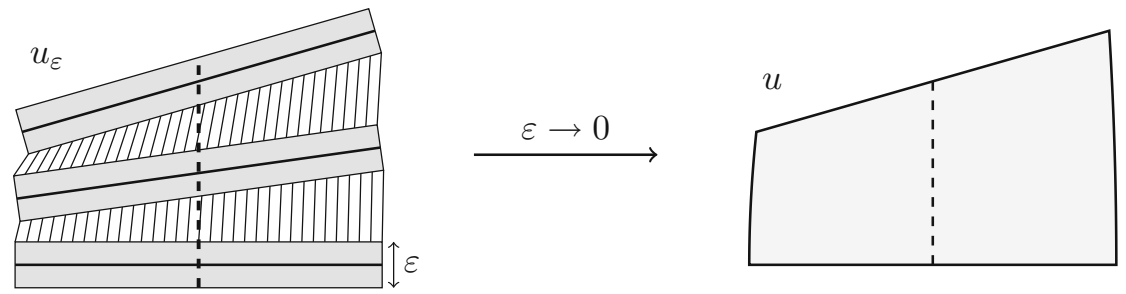

Fig. 5. Illustration of the deformation of Example 2.6 for $n=2$, where the increasing rotation of the stiffer layers is described by $R \in C^{2}\left(\left[-\frac{1}{2}, 2\right] ; S O(2)\right)$ with $R(t) e_{1}=\cos (t) e_{1}+$ $\sin (t) e_{2}$ for $t \in\left[-\frac{1}{2}, 2\right]$

preservation of the limit deformation is necessary to obtain asymptotic rigidity in the sense of Corollary 3.9.

Example 2.6. (Rotation of stiffer layers) Let $R \in C^{1}\left(\left[-\frac{1}{2}, 2\right] ; S O(n)\right)$ with $R e_{i}=$ $e_{i}$ for $i=2, \ldots, n-1$. For each $\varepsilon \in(0,1)$, we set

$$
f_{\varepsilon}(y)=f(y):=\left(y_{1}-\frac{1}{2}\right) R\left(y_{n}\right) e_{1}+\frac{1}{2} e_{1}+\sum_{i=2}^{n} y_{i} e_{i}, \quad y \in \bar{Q},
$$

and take $u_{\varepsilon}$ as defined in Lemma 2.1. Since $\partial_{11} f=0$, it follows from (2.3) that

$$
\int_{\varepsilon Y_{\text {stiff } \cap \Omega}} \operatorname{dist}^{p}\left(\nabla u_{\varepsilon}, S O(n)\right) \mathrm{d} x=0
$$

for any $\varepsilon \in(0,1)$. Moreover, $\nabla u_{\varepsilon} \rightarrow \nabla u=\nabla f$ in $L^{p}\left(\Omega ; \mathbb{R}^{n \times n}\right)$, so that for $x \in \Omega$,

$$
\begin{aligned}
\nabla u(x) & =R\left(x_{n}\right) e_{1} \otimes e_{1}+\sum_{i=2}^{n} e_{i} \otimes e_{i}+\left(x_{1}-\frac{1}{2}\right) R^{\prime}\left(x_{n}\right) e_{1} \otimes e_{n} \\
& =R\left(x_{n}\right)+R^{\prime}\left(x_{n}\right) x \otimes e_{n}+d\left(x_{n}\right) \otimes e_{n},
\end{aligned}
$$

where $d(t)=-\frac{1}{2} R^{\prime}(t) e_{1}-R^{\prime}(t) t e_{n}$ for $t \in(0,1)$. Hence, we obtain

$$
u(x)=R\left(x_{n}\right) x+b\left(x_{n}\right),
$$

with $b(t)=-\frac{1}{2} R e_{1}-\int_{0}^{t} s R^{\prime}(s) e_{n} \mathrm{~d} s+c$ for $t \in(0,1)$ and $c \in \mathbb{R}^{n}$. It is now immediate to see that $u$ has the form stated in Theorem 1.1, but neither is $\operatorname{Re}_{n}$ constant nor is the local volume condition satisfied in general.

In $2 \mathrm{~d}$, this construction corresponds to a $x_{2}$-dependent rotation of the individual stiffer layers around their barycenters, see Fig. 5.

We conclude this section with a reference to Fig. 6, which illustrates at one glance our findings in different scaling regimes for two space dimensions. Notice that any $(2 \times 2)$-matrix can be expressed as $R\left(\beta \mathbb{I}+a \otimes e_{2}\right)$ with $R \in S O(2)$, $\beta \in \mathbb{R}$ and $a \in \mathbb{R}^{2}$. 


\begin{tabular}{|c||l|l|}
\hline & (a) $\nabla u$ & (b) $\nabla u$ with det $\nabla u=1$ \\
\hline \hline$\alpha=0$ & Characterization: & Characterization: \\
& no further restriction on $R, \beta, a$ & $\beta^{2}+\beta a_{2}=1$ \\
\hline$\alpha \in(0, p)$ & Explicit construction: & \\
& $\beta \neq 1$ & \\
& see Example 2.5 & \\
\hline$\alpha=p$ & Explicit construction: & Explicit construction: \\
& $\nabla^{\prime} R=\partial_{1} R \neq 0$ & $R \neq$ const. \\
& see Example 2.3 & see Example 2.4 \\
\hline$\alpha \in(p, \infty)$ & Characterization: & Characterization: \\
& $R \in W^{1, p}(\Omega ; S O(2))$ with $\partial_{1} R=0$, & $R=$ const., $\beta=1$ \\
& $\beta=1, \partial_{1} a=R^{T}$ curl $R$ & $a \| e_{1}, \partial_{1} a=0$ \\
& see Theorem 1.1 & \\
\cline { 2 - 3 } & Explicit construction: & \\
& $R \neq$ const. & \\
& see Example 2.6 & \\
\hline
\end{tabular}

Fig. 6. Overview of the results on the asymptotic behavior of weakly converging sequences $\left(u_{\varepsilon}\right)_{\varepsilon} \subset W^{1, p}\left(\Omega ; \mathbb{R}^{2}\right)$ satisfying (1.3) in the different scaling regimes for $n=2$, a without and $\mathbf{b}$ with local volume constraint on the limit map $u$. It is used here that for any $u \in$ $W^{1, p}\left(\Omega ; \mathbb{R}^{2}\right)$ there are $R \in L^{\infty}(\Omega ; S O(2)), \beta \in L^{p}(\Omega)$ and $a \in L^{p}\left(\Omega ; \mathbb{R}^{2}\right)$ with $\nabla u=$ $R\left(\beta \mathbb{I}+a \otimes e_{2}\right)$

\section{Proof of Necessity in Theorem 1.1}

We will show in this section that weak limits of bounded energy sequences in the context of our model for layered materials with stiff and soft components have a strongly one-dimensional character. To make this more precise, we first introduce the following terminology. A measurable function $f: \Omega \rightarrow \mathbb{R}^{m}$, where $\Omega \subset \mathbb{R}^{n}$ is an open set, is said to be locally one-dimensional in the $e_{n}$-direction if for every $x \in \Omega$ there is an open cuboid $Q_{x} \subset \Omega$ with $x \in Q_{x}$ such that for all $y, z \in Q_{x}$,

$$
f(y)=f(z) \text { if } y_{n}=z_{n} .
$$

We call $f$ (globally) one-dimensional in the $e_{n}$-direction if (3.1) holds for all $y, z \in \Omega$. For $f \in W_{\text {loc }}^{1, p}\left(\Omega ; \mathbb{R}^{m}\right)$ with $p \geqq 1$ local one-dimensionality in the $e_{n^{-}}$ direction of $f$, which means that there exists a representative of $f$ with the property, is equivalent to the condition $\nabla^{\prime} f=0$, as can be seen from a standard mollification argument. Hence, if $\nabla^{\prime} f=0$, the function $f$ can be identified locally (i.e. for any $x \in \Omega$ on an open cuboid $Q_{x} \subset \Omega$ containing $x$ ) with a one-dimensional $W^{1, p}$. function. Since the latter is absolutely continuous, it follows that $f$ is continuous.

The next result and its implications discussed subsequent to its proof generalize the necessity statement of Theorem 1.1 relaxing the assumptions on the domain.

Theorem 3.1. Let $\Omega \subset \mathbb{R}^{n}$ with $n \geqq 2$ be a bounded open set, $1<p<\infty$ and $\alpha>$ p. Furthermore, let $\left(u_{\varepsilon}\right)_{\varepsilon} \subset W^{\overline{1}, p}\left(\Omega ; \mathbb{R}^{n}\right)$ be such that for all $\varepsilon>0$,

$$
\int_{\varepsilon Y_{\text {stiff }} \cap \Omega} \operatorname{dist}^{p}\left(\nabla u_{\varepsilon}, S O(n)\right) \mathrm{d} x \leqq C \varepsilon^{\alpha}
$$


with a constant $C>0$, and $u_{\varepsilon} \rightarrow u$ in $W^{1, p}\left(\Omega ; \mathbb{R}^{n}\right)$ for some $u \in W^{1, p}\left(\Omega ; \mathbb{R}^{n}\right)$.

Then there exist $R \in W_{\mathrm{loc}}^{1, p}(\Omega ; S O(n))$ with $\nabla^{\prime} R=0$ and $b \in W_{\mathrm{loc}}^{1, p}\left(\Omega ; \mathbb{R}^{n}\right)$ with $\nabla^{\prime} b=0$ such that

$$
u(x)=R(x) x+b(x) \text { for } x \in \Omega .
$$

Remark 3.2. (a) Notice that the functions $R$ and $b$ are both locally one-dimensional in the $e_{n}$-direction and continuous. In particular, $u \in C^{0}\left(\Omega ; \mathbb{R}^{n}\right)$.

(b) It is straightforward to generalize Theorem 3.1 to a $(p, q)$-version. Precisely, if $p$ in (3.2) is replaced with any $p<q<\infty$, the same conclusion remains true under the assumption that $\alpha>q$, cf. [15, Section 3.3]. For a discussion of the case $p=1$, see Remark 3.5

(c) One can show that the statement of Theorem 3.1 remains true if the relative thickness of the softer layers $\lambda \in(0,1)$ depends on $\varepsilon$ (then denoted by $\lambda_{\varepsilon}$ ) in such a way that $1-\lambda_{\varepsilon} \gg \varepsilon^{\frac{\alpha}{p}-1}$. For more details, we refer to [15, Theorem 3.3.1].

Theorem 3.1 builds on two classical results, which we recall here for the readers' convenience. The first one is the quantified rigidity result for Sobolev functions established in [33, Theorem 3.1], cf. also [19,22,23] for generalizations to other $W^{1, p}$-settings.

Theorem 3.3. (Quantitative rigidity estimate) Let $U \subset \mathbb{R}^{n}$ with $n \geqq 2$ be a bounded Lipschitz domain and $1<p<\infty$. Then there exists a constant $C=C(U, p)>0$ with the property that for each $u \in W^{1, p}\left(U ; \mathbb{R}^{n}\right)$ there is a rotation $R \in S O(n)$ such that

$$
\|\nabla u-R\|_{L^{p}\left(U ; \mathbb{R}^{n \times n}\right)} \leqq C\|\operatorname{dist}(\nabla u, S O(n))\|_{L^{p}(U)} .
$$

A straightforward scaling argument shows that the constant $C$ remains unaffected by uniform scaling and translation of $U$. Applying the above theorem to increasingly thinner domains, however, leads to degenerating constants. If $U=$ $P_{\varepsilon}=O \times \varepsilon I \subset \mathbb{R}^{n}$ with $\varepsilon>0, O \subset \mathbb{R}^{n-1}$ a cube and $I \subset \mathbb{R}$ a bounded open interval one obtains that

$$
C\left(P_{\varepsilon}, p\right)=\varepsilon^{-1} C\left(P_{1}, p\right),
$$

see [34, Section 4] and [15, Section 3.5.1].

The second tool is the Fréchet-Kolmogorov theorem, a compactness result for $L^{p}$-functions, see e.g. [2, Sections 2.15, U.2] and [37]. Here, we will apply it only in the basic version formulated in the next lemma, that is, for families of functions of one real variable with uniformly bounded essential supremum.

Lemma 3.4. Let $J, J^{\prime} \subset \mathbb{R}$ be open, bounded intervals with $J \subset \subset J^{\prime}$ and $1 \leqq$ $p<\infty$. If the sequence $\left(f_{\varepsilon}\right)_{\varepsilon}$ is uniformly bounded in $L^{\infty}\left(J^{\prime} ; \mathbb{R}^{m}\right)$ satisfying

$$
\lim _{|\xi| \rightarrow 0} \sup _{\varepsilon>0} \int_{J}\left|f_{\varepsilon}(t+\xi)-f_{\varepsilon}(t)\right|^{p} \mathrm{~d} t=0,
$$

then $\left(f_{\varepsilon}\right)_{\varepsilon}$ is relatively compact in $L^{p}\left(J ; \mathbb{R}^{m}\right)$. 
Regarding structure, the next proof proceeds along the lines of [16, Proposition 2.1], which, as mentioned in the introduction, constitutes a special case of Theorem 1.1. Yet, the individual steps are more involved and require new, refined arguments to relax the assumption of the stiff layers being fully rigid and to overcome the restriction to two space dimensions.

Proof of Theorem 3.1. Let $Q=O \times J \subset \Omega$ be a cuboid with $O \subset \mathbb{R}^{n-1}$ an open cube of side length $l>0$ and $J \subset \mathbb{R}$ an open interval. Suppose that there exist open intervals $J^{\prime}, J^{\prime \prime}$ with $J \subset \subset J^{\prime} \subset \subset J^{\prime \prime}$ and $Q^{\prime \prime}:=O \times J^{\prime \prime} \subset \Omega$. Moreover, let $Q^{\prime}:=O \times J^{\prime}$. We define horizontal strips by setting

$$
P_{\varepsilon}^{i}=\left(\mathbb{R}^{n-1} \times \varepsilon[i, i+1)\right) \cap Q^{\prime \prime} \text { for } i \in \mathbb{Z} \text { and } \varepsilon>0 \text {. }
$$

The index set $I_{\varepsilon}$ contains all $i \in \mathbb{Z}$ with $\left|P_{\varepsilon}^{i}\right|=\varepsilon|O|$, and we assume $\varepsilon>0$ to be small enough, so that $Q \subset Q^{\prime} \subset \bigcup_{i \in I_{\varepsilon}} P_{\varepsilon}^{i} \subset Q^{\prime \prime}$.

For the proof, it suffices to show the existence of $R \in W^{1, p}(Q ; S O(n))$ and $b \in W^{1, p}\left(Q ; \mathbb{R}^{n}\right)$ with $\nabla^{\prime} R=0$ and $\nabla^{\prime} b=0$ in $Q$, respectively, such that the characterization (3.3) holds for $x \in Q$. Then we can approximate $\Omega$ from inside with overlapping cuboids to obtain the same statements for any compact $K \subset \Omega$. Indeed, the resulting characterizations in terms of $R$ and $b$ coincide on the overlapping parts. Finally, exhausting $\Omega$ with compact nested subsets proves Theorem 3.1 in the stated generality.

In what follows, the constants $C>0$ depend at most on $n, p, \lambda, \Omega$ and $c$ from (3.2), in particular, they are independent of $\varepsilon, l$ and $J$.

Step 1: Layerwise approximation by rigid body motions In this first step, we will construct a sequence of piecewise affine functions $\left(w_{\varepsilon}\right)_{\varepsilon}$ such that the restriction of each $w_{\varepsilon}$ to a strip $P_{\varepsilon}^{i}$ is a rigid body motion and

$$
\lim _{\varepsilon \rightarrow 0}\left\|u_{\varepsilon}-w_{\varepsilon}\right\|_{L^{p}\left(Q^{\prime} ; \mathbb{R}^{n}\right)}=0
$$

Applying Theorem 3.3 (under consideration of the scaling behavior of the constant according to (3.4)) to the individual stiff layers yields the existence of $C>0$ and of rotations $R_{\varepsilon}^{i} \in S O(n)$ for every $i \in I_{\varepsilon}$ such that

$$
\left\|\nabla u_{\varepsilon}-R_{\varepsilon}^{i}\right\|_{L^{p}\left(\varepsilon Y_{\text {stiff }} \cap P_{\varepsilon}^{i} ; \mathbb{R}^{n \times n}\right)} \leqq C \varepsilon^{-1}\left\|\operatorname{dist}\left(\nabla u_{\varepsilon}, S O(n)\right)\right\|_{L^{p}\left(\varepsilon Y_{\text {stiff }} \cap P_{\varepsilon}^{i}\right)} .
$$

Let $w_{\varepsilon} \in L^{\infty}\left(Q^{\prime} ; \mathbb{R}^{n}\right)$ be defined by $w_{\varepsilon}=\sigma_{\varepsilon}+b_{\varepsilon}$, where

$$
\sigma_{\varepsilon}(x)=\sum_{i \in I_{\varepsilon}}\left(R_{\varepsilon}^{i} x\right) \mathbb{1}_{P_{\varepsilon}^{i} \cap Q^{\prime}}(x), \quad x \in Q^{\prime},
$$

and

$$
b_{\varepsilon}=\sum_{i \in I_{\varepsilon}} b_{\varepsilon}^{i} \mathbb{1}_{P_{\varepsilon}^{i} \cap Q^{\prime}} \quad \text { with } b_{\varepsilon}^{i}=f_{\varepsilon Y_{\text {stiff }} \cap P_{\varepsilon}^{i}} u_{\varepsilon}-R_{\varepsilon}^{i} x \mathrm{~d} x
$$


The specific choice of the values $b_{\varepsilon}^{i}$ implies that $\int_{\varepsilon Y_{\text {stiff }} \cap P_{\varepsilon}^{i}} u_{\varepsilon}-w_{\varepsilon} \mathrm{d} x=0$, and therefore allows us to apply Poincaré's inequality to $u_{\varepsilon}-w_{\varepsilon}$ on each stiff layer. Hence, one obtains for every $i \in I_{\varepsilon}$ that

$$
\left\|u_{\varepsilon}-w_{\varepsilon}\right\|_{L^{p}\left(\varepsilon Y_{\text {stiff }} \cap P_{\varepsilon}^{i} ; \mathbb{R}^{n}\right)} \leqq C\left\|\nabla u_{\varepsilon}-R_{\varepsilon}^{i}\right\|_{L^{p}\left(\varepsilon Y_{\text {stiff }} \cap P_{\varepsilon}^{i} ; \mathbb{R}^{n \times n}\right)},
$$

see e.g. [35, Section 7.8] for details on the domain dependence of the Poincare constant.

Next we derive a corresponding bound on the softer layers. By a shifting argument, this problem can be reduced to estimate (3.8) for the stiff layers. The error is given in terms of difference quotients in the $e_{n}$-direction of $u_{\varepsilon}-w_{\varepsilon}$, which we control uniformly. More precisely, for fixed $i \in I_{\varepsilon}$ we cover $\varepsilon Y_{\text {soft }} \cap P_{\varepsilon}^{i}$ with finitely many shifted copies of $\varepsilon Y_{\text {stiff }} \cap P_{\varepsilon}^{i}$, that is, we choose $0<\delta_{\varepsilon, k} \leqq \lambda \varepsilon$ for $k=1, \ldots, N:=\left\lceil\frac{\lambda}{1-\lambda}\right\rceil$ such that the $\delta_{\varepsilon, k}$-shifted stiff layers $O_{\varepsilon, k}^{i}:=\left(\varepsilon Y_{\text {stiff }} \cap\right.$ $\left.P_{\varepsilon}^{i}\right)-\delta_{\varepsilon, k} e_{n}$ satisfy $\varepsilon Y_{\text {soft }} \cap P_{\varepsilon}^{i} \subset \bigcup_{k=1}^{N} O_{\varepsilon, k}^{i}$. Then,

$$
\begin{aligned}
\int_{O_{\varepsilon, k}^{i}}\left|u_{\varepsilon}-w_{\varepsilon}\right|^{p} \mathrm{~d} x \leqq & C \int_{\varepsilon Y_{\text {stiff }} \cap P_{\varepsilon}^{i}}\left|u_{\varepsilon}-w_{\varepsilon}\right|^{p} \mathrm{~d} x \\
& +C \int_{\varepsilon Y_{\text {stiff } \cap P_{\varepsilon}^{i}}}\left|\left(u_{\varepsilon}-w_{\varepsilon}\right)(x)-\left(u_{\varepsilon}-w_{\varepsilon}\right)\left(x-\delta_{\varepsilon, k} e_{n}\right)\right|^{p} \mathrm{~d} x \\
\leqq & C\left(\left\|u_{\varepsilon}-w_{\varepsilon}\right\|_{L^{p}\left(\varepsilon Y_{\text {stiff }} \cap P_{\varepsilon}^{i} ; \mathbb{R}^{n}\right)}^{p}+\delta_{\varepsilon, k}^{p}\left\|\partial_{n} u_{\varepsilon}-\partial_{n} w_{\varepsilon}\right\|_{L^{p}\left(P_{\varepsilon}^{i} ; \mathbb{R}^{n}\right)}^{p}\right) .
\end{aligned}
$$

Here, we have used a one-dimensional difference quotient estimate with respect to the $x_{n}$-variable. Summing over the $N$ covering cuboids then leads to

$$
\begin{aligned}
& \int_{\varepsilon Y_{\text {soft }} \cap P_{\varepsilon}^{i}}\left|u_{\varepsilon}-w_{\varepsilon}\right|^{p} \mathrm{~d} x \\
& \quad \leqq C\left(\left\|u_{\varepsilon}-w_{\varepsilon}\right\|_{L^{p}\left(\varepsilon Y_{\text {stiff }}^{p} \cap P_{\varepsilon}^{i} ; \mathbb{R}^{n}\right)}^{p}+\varepsilon^{p}\left\|\nabla u_{\varepsilon}\right\|_{L^{p}\left(P_{\varepsilon}^{i} ; \mathbb{R}^{n \times n}\right)}^{p}+\varepsilon^{p}\left|P_{\varepsilon}^{i}\right|\right)
\end{aligned}
$$

notice that the last term results from the fact that $\nabla w_{\varepsilon} \in S O(n)$ on $P_{\varepsilon}^{i}$ by (3.7).

Finally, we take the sum over $i \in I_{\varepsilon}$ to deduce from (3.8) and (3.6) that

$$
\begin{aligned}
& \int_{Q^{\prime}}\left|u_{\varepsilon}-w_{\varepsilon}\right|^{p} \mathrm{~d} x \\
& \quad \leqq C\left(\varepsilon^{-p} \| \operatorname{dist}\left(\nabla u_{\varepsilon}, S O(n)\left\|_{L^{p}\left(\varepsilon Y_{\text {stiff } \cap \Omega)}\right.}^{p}+\varepsilon^{p}\right\| u_{\varepsilon} \|_{W^{1, p}\left(\Omega ; \mathbb{R}^{n}\right)}^{p}+\varepsilon^{p}|\Omega|\right) .\right.
\end{aligned}
$$

Therefore, by (3.2) and the uniform boundedness of $\left(u_{\varepsilon}\right)_{\varepsilon}$ in $W^{1, p}\left(\Omega ; \mathbb{R}^{n}\right)$,

$$
\left\|u_{\varepsilon}-w_{\varepsilon}\right\|_{L^{p}}\left(Q^{\prime} ; \mathbb{R}^{n}\right) \leqq C\left(\varepsilon^{\frac{\alpha}{p}-1}+\varepsilon\right) .
$$

Since $\alpha>p$, this implies (3.5).

Step 2: Compactness of the approximating rigid body motions Consider for $\varepsilon>0$ the piecewise constant one-dimensional auxiliary function $\Sigma_{\varepsilon}: J^{\prime} \rightarrow S O(n)$ defined by

$$
\Sigma_{\varepsilon}(t)=\sum_{i \in I_{\varepsilon}} R_{\varepsilon}^{i} \mathbb{1}_{\varepsilon[i, i+1)}(t), \quad t \in J^{\prime},
$$


with $R_{\varepsilon}^{i}$ as in Step 1. In relation to (3.7), it holds that $\sigma_{\varepsilon}(x)=\Sigma_{\varepsilon}\left(x_{n}\right) x$ for $x \in Q^{\prime}$.

Step 2a: Estimate for rotations on different strips Next we will show that for every $\xi \in \mathbb{R}$ such that $J \cup(J+\xi) \subset J^{\prime}$,

$$
\begin{aligned}
& \left\|\Sigma_{\varepsilon}(\cdot+\xi)-\Sigma_{\varepsilon}\right\|_{L^{p}\left(J ; \mathbb{R}^{n \times n}\right)} \\
& \quad \leqq C l^{-p-n+1}\left(\left\|u_{\varepsilon}-w_{\varepsilon}\right\|_{L^{p}\left(Q^{\prime} ; \mathbb{R}^{n}\right)}+\xi\left\|u_{\varepsilon}\right\|_{W^{1, p}\left(\Omega ; \mathbb{R}^{n}\right)}\right) .
\end{aligned}
$$

To this end, we estimate the expression $\left\|w_{\varepsilon}\left(\cdot+\xi e_{n}\right)-w_{\varepsilon}\right\|_{L^{p}}\left(Q ; \mathbb{R}^{n}\right)$ from above and below.

The upper bound follows from

$$
\begin{aligned}
& \left\|w_{\varepsilon}\left(\cdot+\xi e_{n}\right)-w_{\varepsilon}\right\|_{L^{p}\left(Q ; \mathbb{R}^{n}\right)} \\
& \quad \leqq \\
& \quad\left\|w_{\varepsilon}-u_{\varepsilon}\right\|_{L^{p}}\left(Q^{\prime} ; \mathbb{R}^{n}\right)+\left\|w_{\varepsilon}\left(\cdot+\xi e_{n}\right)-u_{\varepsilon}\left(\cdot+\xi e_{n}\right)\right\|_{L^{p}\left(Q ; \mathbb{R}^{n}\right)} \\
& \quad+\left\|u_{\varepsilon}\left(\cdot+\xi e_{n}\right)-u_{\varepsilon}\right\|_{L^{p}}\left(Q ; \mathbb{R}^{n}\right) \\
& \leqq 2\left\|w_{\varepsilon}-u_{\varepsilon}\right\|_{L^{p}\left(Q^{\prime} ; \mathbb{R}^{n}\right)}+\xi\left\|\partial_{n} u_{\varepsilon}\right\|_{L^{p}\left(Q^{\prime} ; \mathbb{R}^{n}\right) .}
\end{aligned}
$$

For the lower bound, we set $d_{\varepsilon, \xi}^{i}=b_{\varepsilon}^{i+\left\lfloor\frac{\xi}{\varepsilon}\right\rfloor}-b_{\varepsilon}^{i}+\xi R_{\varepsilon}^{i+\left\lfloor\frac{\xi}{\varepsilon}\right\rfloor} e_{n}$ and use Lemma A.1 to derive that

$$
\begin{aligned}
& \left\|w_{\varepsilon}\left(\cdot+\xi e_{n}\right)-w_{\varepsilon}\right\|_{L^{p}\left(Q ; \mathbb{R}^{n}\right)}^{p}=\sum_{i \in I_{\varepsilon}} \int_{P_{\varepsilon}^{i} \cap Q}\left|\left(R_{\varepsilon}^{i+\left\lfloor\frac{\xi}{\varepsilon}\right\rfloor}-R_{\varepsilon}^{i}\right) x+d_{\varepsilon, \xi}^{i}\right|^{p} \mathrm{~d} x \\
& \geqq C l^{p} \sum_{i \in I_{\varepsilon}}\left|R_{\varepsilon}^{i+\left\lfloor\frac{\xi}{\varepsilon}\right\rfloor}-R_{\varepsilon}^{i}\right|^{p}\left|P_{\varepsilon}^{i} \cap B^{\prime}\right| \geqq C l^{p}\left\|\Sigma_{\varepsilon}(\cdot+\xi)-\Sigma_{\varepsilon}\right\|_{L^{p}\left(J ; \mathbb{R}^{n \times n}\right)}^{p} .
\end{aligned}
$$

Combining (3.12) and (3.13) gives (3.11).

Step 2b: Application of the Fréchet-Kolmogorov theorem To establish strong $L^{p}$-convergence of $\left(\Sigma_{\varepsilon}\right)_{\varepsilon}$ as $\varepsilon \rightarrow 0$, observe that in view of (3.9) and the uniform boundedness of $\left(u_{\varepsilon}\right)_{\varepsilon}$ in $W^{1, p}\left(\Omega ; \mathbb{R}^{n}\right)$, estimate (3.11) turns into

$$
\left\|\Sigma_{\varepsilon}(\cdot+\xi)-\Sigma_{\varepsilon}\right\|_{L^{p}\left(J ; \mathbb{R}^{n \times n}\right)} \leqq C l^{-p-n+1}\left(\xi+\varepsilon^{\frac{\alpha}{p}-1}\right) .
$$

It is standard to verify (see e.g. [33, Proof of Theorem 4.1] for an analogous argument) that then

$$
\limsup _{|\xi| \rightarrow 0} \sup _{\varepsilon>0}\left\|\Sigma_{\varepsilon}(\cdot+\xi)-\Sigma_{\varepsilon}\right\|_{L^{p}\left(J ; \mathbb{R}^{n \times n}\right)}=0 .
$$

Hence, by Theorem 3.4, there exist a subsequence (not relabeled) and a $\Sigma_{0} \in$ $L^{p}\left(J ; \mathbb{R}^{n \times n}\right)$ such that

$$
\Sigma_{\varepsilon} \rightarrow \Sigma_{0} \text { in } L^{p}\left(J ; \mathbb{R}^{n \times n}\right) .
$$

Note that $\Sigma_{0}$ may still depend on the subsequence at this point. In Step 3, $\Sigma_{0}$ will be characterized in terms of the limit function $u$, which makes $\Sigma_{0}$ unique and the above argument independent of the choice of subsequences. Due to the strong $L^{p}$ convergence of $\left(\Sigma_{\varepsilon}\right)_{\varepsilon}$, which preserves lengths and angles almost everywhere, we conclude that $\Sigma_{0} \in S O(n)$ a.e. in $J^{\prime}$. 
Step 2c: Regularity of $\Sigma_{0}$ As a result of (3.14), we obtain an estimate on the difference quotients of $\Sigma_{\varepsilon}$, precisely

$$
\int_{J}\left|\frac{\Sigma_{\varepsilon}(t+\xi)-\Sigma_{\varepsilon}(t)}{\xi}\right|^{p} \mathrm{~d} t \leqq C l^{-p-n+1}\left(1+\xi^{-p} \varepsilon^{\alpha-p}\right) .
$$

Passing to the limit $j \rightarrow \infty$ results in

$$
\int_{J}\left|\frac{\Sigma_{0}(t+\xi)-\Sigma_{0}(t)}{\xi}\right|^{p} \mathrm{~d} t \leqq C l^{-p-n+1}
$$

which shows that $\Sigma_{0} \in W^{1, p}\left(J ; \mathbb{R}^{n \times n}\right)$, see e.g. [44, Theorem 10.55].

Step 3: Representation of the limit function $u$ Recall the definitions of $\sigma_{\varepsilon}$ in (3.7) and $\Sigma_{\varepsilon}$ in (3.10). With $\sigma_{0}(x)=\Sigma_{0}\left(x_{n}\right) x$ for $x \in Q$ one has that

$$
\int_{Q}\left|\sigma_{\varepsilon}-\sigma_{0}\right|^{p} \mathrm{~d} x \leqq \sum_{i \in I_{\varepsilon}} \int_{P_{\varepsilon} \cap Q}\left|R_{\varepsilon}^{i}-\Sigma_{0}\left(x_{n}\right)\right|^{p}|x|^{p} \mathrm{~d} x \leqq C \int_{J}\left|\Sigma_{\varepsilon}-\Sigma_{0}\right|^{p} \mathrm{~d} t,
$$

Then, by (3.15),

$$
\sigma_{\varepsilon} \rightarrow \sigma_{0} \quad \text { in } L^{p}\left(Q ; \mathbb{R}^{n}\right)
$$

Since $b_{\varepsilon}=w_{\varepsilon}-\sigma_{\varepsilon}=\left(w_{\varepsilon}-u_{\varepsilon}\right)+u_{\varepsilon}-\sigma_{\varepsilon}$ we find in view of (3.5), (3.17) and the convergence $u_{\varepsilon} \rightarrow u$ in $L^{p}\left(\Omega ; \mathbb{R}^{n}\right)$ by the compact embedding of $W^{1, p}\left(Q ; \mathbb{R}^{n}\right)$ into $L^{p}\left(Q ; \mathbb{R}^{n}\right)$ that

$$
b_{\varepsilon} \rightarrow u-\sigma_{0}=: b \text { in } L^{p}\left(Q ; \mathbb{R}^{n}\right) .
$$

Due to the regularity of $u$ and $\sigma_{0}$, it follows that $b \in W^{1, p}\left(Q ; \mathbb{R}^{n}\right)$. Since $b_{\varepsilon}$ is independent of the $x^{\prime}$-variables, the same is true for $b$. Finally, defining

$$
R(x)=\Sigma_{0}\left(x_{n}\right) \quad \text { for } x \in Q,
$$

proves the desired representation of $u$.

Remark 3.5. (a) Setting $p=1$ in Remark 3.2b) in combination with Theorem 3.1 leads to the representation (3.3) with $R \in B V_{\text {loc }}(\Omega ; S O(n))$ and $b \in B V_{\text {loc }}\left(\Omega ; \mathbb{R}^{n}\right)$ satisfying $D^{\prime} R=0$ and $D^{\prime} b=0$, respectively. The reasoning is the same as for $p>1$, but instead of getting $\Sigma_{0} \in W^{1,1}\left(J ; \mathbb{R}^{n \times n}\right)$ from (3.16), we can only deduce that $\Sigma_{0} \in B V\left(J ; \mathbb{R}^{n \times n}\right)$, see e.g. [44, Corollary 2.43].

(b) Notice that in view of (3.18) and (3.16) it holds that

$$
\|R\|_{W^{1, p}\left(Q ; \mathbb{R}^{n \times n}\right)}^{p} \leqq C\left(1+l^{-p}\right) .
$$

This estimate is not uniform for all cuboids $Q \subset \Omega$ as used in the proof of Theorem 3.1. In fact, the bound becomes large for cuboids with small crosssection. One can therefore not expect in general that the weak derivatives of $R$ be $p$-integrable on the whole of $\Omega$. 
(a)

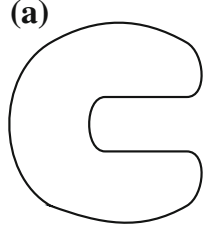

(b)

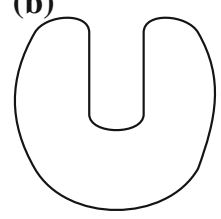

(c)

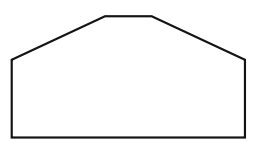

(d)

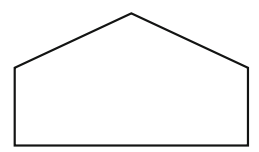

Fig. 7. Illustration of Definitions 3.6 and 3.7: examples of bounded Lipschitz domains that are $\mathbf{a}$ cross-section connected, $\mathbf{b}$ not cross-section connected, $\mathbf{c}$ flat and $\mathbf{d}$ not flat

(c) If $\Omega$ in Theorem 3.1 is of the form $\Omega=O \times I$ with $O \subset \mathbb{R}^{n-1}$ an open cube of side length $l>0$ and $I \subset \mathbb{R}$ an interval, then the proof shows that $R \in W^{1, p}(\Omega ; S O(n))$, and hence also $b \in W^{1, p}\left(\Omega ; \mathbb{R}^{n}\right)$, for any $p>1$.

Indeed, let us choose intervals $J_{k} \subset \subset I$ for $k \in \mathbb{N}$ such that $J_{k} \subset J_{k+1}$ and $I=\bigcup_{k=1}^{\infty} J_{k}$ and set $Q_{k}=O \times J_{k}$. Then by estimate (3.19),

$$
\|R\|_{W^{1, p}\left(Q_{k} ; \mathbb{R}^{n \times n}\right)}^{p} \leqq C,
$$

with $C>0$ independent of $k$. Since the cuboids $Q_{k}$ exhaust $\Omega$, the uniform bound (3.20) yields that $R \in W^{1, p}(\Omega ; S O(n))$.

The observation of Remark 3.5(c) can be extended to a larger class of Lipschitz domains. In fact, under suitable additional assumptions on $\Omega$, namely connectedness of cross-sections and a flatness property, which are introduced in Definitions 3.6 and 3.7, we can drop the restriction to local $W^{1, p}$-regularity of $R$ and $b$ in Theorem 3.1, as Corollary 3.8 below shows.

Definition 3.6. (Connectedness of cross-sections) An open set $\Omega \subset \mathbb{R}^{n}$ is called cross-section connected if for any $t \in \mathbb{R}$ the intersection $\Omega_{t}$ of $\Omega$ with the hyperplane $H_{t}=\left\{x \in \mathbb{R}^{n}: x_{n}=t\right\}$ is connected.

Clearly, every convex set is cross-section connected, but also cylinders and cones in $\mathbb{R}^{n}$ (oriented in the $e_{n}$-direction) with non-convex cross section are. In Fig. 7a, b we give a two-dimensional example for illustration. An important property of domains $\Omega$ as in Definition 3.6 is that any locally one-dimensional vector (and matrix) field in the $e_{n}$-direction defined on $\Omega$ is already globally one-dimensional in the $e_{n}$-direction, cf. Lemma A.2.

Definition 3.7. (Flatness) We call an open set $\Omega \subset \mathbb{R}^{n}$ flat, if for all $t \in \mathbb{R}$ the intersection of $\bar{\Omega}$ with the hyperplane $H_{t}=\left\{x \in \mathbb{R}^{n}: x_{n}=t\right\}$ is either empty or has nonempty relative interior.

The intuitive geometric interpretation of flatness of bounded domains is that it rules out sets with sharp or rounded corners and peaks pointing in the direction of $e_{n}$. Simple examples include cylinders with axis parallel to $e_{n}$, whereas cones with the same orientation are not flat, see also Fig. 7c, d. A bounded Lipschitz domain $\Omega \subset \mathbb{R}^{n}$ does in general not satisfy the condition of Definition (3.7), but it can be turned into a flat Lipschitz domain by cutting it off on top and bottom, i.e., by taking $\left(\mathbb{R}^{n-1} \times(a, b)\right) \cap \Omega$, where $a, b \in \mathbb{R}$ with $a<b$ are such that the cross sections $\Omega_{a}$ and $\Omega_{b}$ are non-empty. 
Corollary 3.8. In addition to the assumptions of Theorem 3.1 , let $\Omega \subset \mathbb{R}^{n}$ be a flat and cross-section connected Lipschitz domain. Then the representation (3.3) holds with $R \in W^{1, p}(\Omega ; S O(n))$ and $b \in W^{1, p}\left(\Omega ; \mathbb{R}^{n}\right)$.

Proof. Let $Q_{\Omega}$ be the smallest open cuboid containing $\Omega$ and let $a, b \in \mathbb{R}$ with $a<b$ and $O_{\Omega} \subset \mathbb{R}^{n-1}$ be such that $Q_{\Omega}=O_{\Omega} \times J_{\Omega}$ with $J_{\Omega}=(a, b)$. We observe first that due to the connectedness of the cross-sections of $\Omega$, the map $R$ from (3.3) is globally one-dimensional in the $e_{n}$-direction and can thus be identified with a onedimensional function $\Sigma \in W_{\mathrm{loc}}^{1, p}\left(J_{\Omega} ; S O(n)\right)$, see Lemma A.2 and Remark A.3.

Moreover, since $\Omega$ is a flat Lipschitz domain there exist $x_{a} \in \Omega_{a}$ and $x_{b} \in \Omega_{b}$ along with open cuboids $Q_{a}=O \times(a, a+r)$ and $Q_{b}=O \times(b-r, b)$ of height $r>0$ and cross-section $O \subset \mathbb{R}^{n-1}$ such that $Q_{a} \cap Q_{\Omega} \subset \Omega$ and $Q_{b} \cap Q_{\Omega} \subset \Omega$. Applying Remark 3.5(c) to the restrictions $R_{a}=\left.R\right|_{Q_{a}}$ and $R_{b}=\left.R\right|_{Q_{b}}$ gives that $R_{a} \in W^{1, p}\left(Q_{a} ; S O(n)\right)$ and $R_{b} \in W^{1, p}\left(Q_{b} ; S O(n)\right)$, which correspond to elements in $\Sigma_{a} \in W^{1, p}(a, a+r ; S O(n))$ and $\Sigma_{b} \in W^{1, p}(b-r, b ; S O(n))$, respectively. Hence, $\Sigma \in W^{1, p}\left(J_{\Omega} ; S O(n)\right)$ and $R \in W^{1, p}\left(Q_{\Omega} ; S O(n)\right)$, thus also $R \in W^{1, p}(\Omega ; S O(n))$.

Since $b=u-R x$ with $u \in W^{1, p}\left(\Omega ; \mathbb{R}^{m}\right)$, one immediately gets the desired statement for $b$.

We conclude this section with the following specialization of Corollary 3.8, which involves the additional condition that the limit map is locally volume preserving.

Corollary 3.9. In addition to the assumptions on $\Omega,\left(u_{\varepsilon}\right)_{\varepsilon}$ and $u$ in Corollary 3.8, let $u \in W^{1, r}\left(\Omega ; \mathbb{R}^{n}\right)$ for $r \geqq n$ be such that $\operatorname{det} \nabla u=1$ a.e. in $\Omega$.

Then the limit representation in (3.3) holds with $R_{n}$ constant. If $\Omega$ is simply connected, one has in particular that

$$
\nabla u=Q S\left(\mathbb{I}+a \otimes e_{n}\right),
$$

where $Q \in S O(n), S=\operatorname{diag}\left(S^{\prime}, 1\right)$ with $S^{\prime} \in W^{1, p}(\Omega ; S O(n-1))$ satisfying $\nabla^{\prime} S^{\prime}=0$ and $a \in L^{\max \{r, p\}}\left(\Omega ; \mathbb{R}^{n}\right)$ with $D^{\prime} a=\left(S^{\prime}\right)^{T}\left(\partial_{n} S^{\prime}\right)$ and $a_{n}=0$.

Proof of Corollary 3.9. By Theorem 3.1, we know that $u$ has the representation (3.3). Hence,

$$
\nabla u=R+\left(\partial_{n} R\right) x \otimes e_{n}+\partial_{n} b \otimes e_{n}=R+\tilde{a} \otimes e_{n}=R\left(\mathbb{I}+a \otimes e_{n}\right)
$$

with $\tilde{a}=\left(\partial_{n} R\right) x+\partial_{n} b$ and $a=R^{T} \tilde{a}$. Since det $\nabla u=\operatorname{det}\left(R\left(\mathbb{I}+a \otimes e_{n}\right)\right)=1+a_{n}$, we conclude in view of the local volume preservation constraint that $a_{n}=0$.

Differentiating the identity $0=a_{n}=\tilde{a} \cdot R e_{n}$ with respect to the $i$ th variable for $i \in\{1, \ldots, n-1\}$, while taking into account that $\nabla^{\prime} R=0$ and $\nabla^{\prime} b=0$, implies that $\partial_{n}\left(R e_{i}\right) \cdot R e_{n}=0$. Since $R e_{i}$ is orthogonal on $R e_{n}$ pointwise almost everywhere, it follows from the product rule that

$$
\partial_{n}\left(R e_{n}\right) \cdot R e_{i}=0 \text { for } i=1, \ldots, n-1 .
$$


Together with

$$
0=\frac{1}{2} \partial_{n}\left|R e_{n}\right|^{2}=\partial_{n}\left(R e_{n}\right) \cdot R e_{n},
$$

we obtain that $\partial_{n}\left(R e_{n}\right)=0$. Hence, $R e_{n}$ is constant, and $R$ splits multiplicatively into the product of $Q$ and $S$ as in the statement.

Finally, the restriction on the distributional derivatives of $a$ with respect to the first $n-1$ variables follows via straightforward calculation from the gradient structure of $\nabla u$, which requires that $\operatorname{curl} \nabla u=0$.

Remark 3.10. If $n=2$, the gradient representation of $u$ in (3.21) becomes

$$
\nabla u=Q\left(\mathbb{I}+\gamma e_{1} \otimes e_{2}\right),
$$

with $Q \in S O(2)$ and $\gamma \in L^{p}(\Omega)$ with $\partial_{1} \gamma=0$, cf. also [16, Proposition 2.1]. In the two-dimensional setting, the class of limit deformations $u$ of $\Omega$ is highly restricted, in fact, only horizontal shearing and global rotation can occur.

\section{Sufficiency Statement in Theorem 1.1}

Our starting point in this section are functions $u \in W^{1, p}\left(\Omega ; \mathbb{R}^{n}\right)$ with gradients of the form

$$
\nabla u(x)=R(x)+\partial_{n} R(x) x \otimes e_{n}+d(x) \otimes e_{n}, \quad x \in \Omega,
$$

where $R \in W^{1, p}(\Omega ; S O(n))$ and $d \in L^{p}\left(\Omega ; \mathbb{R}^{n}\right)$ with $\nabla^{\prime} R=0$ and $D^{\prime} d=0$, respectively. If not mentioned otherwise, $1<p<\infty$ and $\Omega \subset \mathbb{R}^{n}$ is a bounded domain.

We will show how such $u$ (under suitable technical assumptions) can be approximated in the sense of weak convergence in $W^{1, p}\left(\Omega ; \mathbb{R}^{n \times n}\right)$ by functions $u_{\varepsilon}$ that are defined on a layered domain with length scale of oscillations $\varepsilon$ and coincide with rigid body motions on the stiff components. This in particular proves Theorem 1.1(ii).

Before stating the general result, let us consider a simple example for motivation. If $u$ is affine, then $\nabla u=F$ for some $F \in \mathbb{R}^{n \times n}$ and there exist a matrix $R_{F} \in S O(n)$ and a vector $d_{F} \in \mathbb{R}^{n}$ such that $\nabla u=F=R_{F}+d_{F} \otimes e_{n}$. This motivates the definition

$$
\mathcal{A}=\left\{F \in \mathbb{R}^{n \times n}: F=R_{F}+d_{F} \otimes e_{n} \text { with } R_{F} \in S O(n) \text { and } d_{F} \in \mathbb{R}^{n}\right\} .
$$

Moreover, we set

$$
F_{\lambda}=R_{F}+\frac{1}{\lambda} d_{F} \otimes e_{n} \text { for } F \in \mathcal{A}
$$

In the affine case, the construction of a suitable approximation is particularly simple. The idea is to compensate for the stiff layers by performing stronger deformations 
on the softer layers, which leads to the following laminate construction: for $\varepsilon>0$, let $v_{\varepsilon}^{F} \in W^{1, \infty}\left(\Omega ; \mathbb{R}^{n}\right)$ be such that

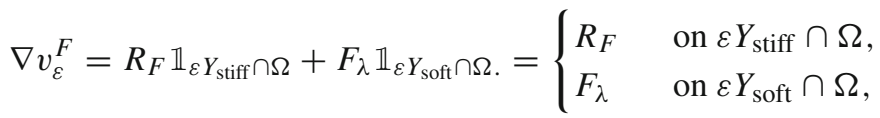

then $\nabla v_{\varepsilon}^{F} \in S O(n)$ a.e. in $\varepsilon Y_{\text {stiff }} \cap \Omega$ and $\nabla v_{\varepsilon}^{F}=R+\frac{1}{\lambda} \mathbb{1}_{\varepsilon} Y_{\text {soft }} d \otimes e_{n} \rightarrow \nabla u$ in $L^{p}\left(\Omega ; \mathbb{R}^{n \times n}\right)$ as a consequence of the weak convergence of highly oscillating sequences (see e.g. [18, Section 2.3]). Finally, we set $u_{\varepsilon}=v_{\varepsilon}^{F}$ for all $\varepsilon$ to obtain the desired approximating functions in this special case.

The construction behind the general approximation result is inspired by the case of affine limits. In view of (4.3), we have

$$
(\nabla u)_{\lambda}(x)=(\nabla u(x))_{\lambda}=R(x)+\frac{1}{\lambda}\left(\partial_{n} R\right)(x) x \otimes e_{n}+\frac{1}{\lambda} d(x) \otimes e_{n}, \quad x \in \Omega .
$$

Proposition 4.1. Let $\Omega \subset \mathbb{R}^{n}$ be a bounded, flat and cross-section connected Lipschitz domain and let $u \in W^{1, p}\left(\Omega ; \mathbb{R}^{n}\right)$ with $\nabla u$ as in (4.1). Then there exists a sequence $\left(u_{\varepsilon}\right)_{\varepsilon} \subset W^{1, p}\left(\Omega ; \mathbb{R}^{n}\right)$ with $\nabla u_{\varepsilon} \in S O(n)$ a.e. in $\varepsilon Y_{\text {stiff }} \cap \Omega$ such that $\nabla u_{\varepsilon} \rightarrow \nabla u$ in $L^{p}\left(\Omega ; \mathbb{R}^{n \times n}\right)$.

More specifically, there is $\left(R_{\varepsilon}\right)_{\varepsilon} \subset W^{1, p}(\Omega ; S O(n))$ with $\nabla^{\prime} R_{\varepsilon}=0$ on $\Omega$ and $\partial_{n} R_{\varepsilon}=0$ on $\varepsilon Y_{\text {stiff }} \cap \Omega$ such that

$$
\nabla u_{\varepsilon}=R_{\varepsilon} \text { in } \varepsilon Y_{\text {stiff }} \cap \Omega,
$$

and

$R_{\varepsilon} \rightarrow R$ in $W^{1, p}\left(\Omega ; \mathbb{R}^{n \times n}\right)$ and $\left\|\nabla u_{\varepsilon}-(\nabla u)_{\lambda}\right\|_{L^{p}\left(\varepsilon Y_{\text {soft }} \cap \Omega ; \mathbb{R}^{n \times n}\right)} \rightarrow 0$ as $\varepsilon \rightarrow 0$.

Remark 4.2. The same result still holds also under relaxed conditions on a bounded Lipschitz domain $\Omega$, namely when $\Omega$ can be partitioned into finitely many components that are flat and cross-section connected. More details can be found in [15, Section 4.2].

Proof. Let $Q_{\Omega}$ denote the smallest cuboid containing $\Omega$. By (4.1) and Lemma A.2 (see also Remark A.3(b)), we may assume after constant extension orthogonal to $e_{n}$ that $R \in W^{1, p}\left(Q_{\Omega} ; S O(n)\right)$ is globally one-dimensional in the $e_{n}$-direction and continuous. Upon writing $Q_{\Omega}=O_{\Omega} \times J_{\Omega}$ with $O_{\Omega} \subset \mathbb{R}^{n-1}$ an open cuboid and $J_{\Omega} \subset \mathbb{R}$ an open, bounded interval, there is a one-dimensional function $\Sigma \in$ $W^{1, p}\left(J_{Q} ; S O(n)\right)$ such that $R(x)=\Sigma\left(x_{n}\right)$ for $x \in Q_{\Omega}$.

Let $\left(\Sigma_{\varepsilon}\right)_{\varepsilon} \subset W^{1, p}\left(J_{\Omega} ; S O(n)\right)$ be the approximating sequence for $\Sigma$ resulting from Lemma 4.3 below, that is, $\Sigma_{\varepsilon} \rightarrow \Sigma$ in $W^{1, p}\left(J_{\Omega} ; \mathbb{R}^{n \times n}\right)$ and $\Sigma_{\varepsilon}^{\prime}=0$ in $\varepsilon I_{\text {stiff }} \cap J_{\Omega}$. Moreover, the convergence (4.8) holds. We set $R_{\varepsilon}(x)=\Sigma_{\varepsilon}\left(x_{n}\right)$ for $x \in Q_{\Omega}$, so that $R_{\varepsilon} \in W^{1, p}\left(Q_{\Omega} ; S O(n)\right)$ with $\nabla^{\prime} R_{\varepsilon}=0$, and define

$$
U_{\varepsilon}=R_{\varepsilon} \mathbb{1}_{\varepsilon} Y_{\text {stiff }} \cap Q_{\Omega}+U_{\lambda, \varepsilon} \mathbb{1}_{\varepsilon} Y_{\text {soft }} \cap Q_{\Omega},
$$


where

$$
U_{\lambda, \varepsilon}(x)=R_{\varepsilon}(x)+\left(\partial_{n} R_{\varepsilon}\right)(x) x \otimes e_{n}+\frac{1}{\lambda} d(x) \otimes e_{n}, \quad x \in Q_{\Omega} .
$$

We claim that for each $\varepsilon>0$ the function $U_{\varepsilon}$ has gradient structure, meaning that there exists a potential $u_{\varepsilon} \in W^{1, p}\left(Q_{\Omega} ; \mathbb{R}^{n}\right)$ with $\nabla u_{\varepsilon}=U_{\varepsilon}$. To see this, it suffices to show that the distributional curl of $U_{\varepsilon}$ vanishes on $Q_{\Omega}$. We remark that $Q_{\Omega}$ as a cuboid is simply connected. Indeed, let $\varphi \in C_{c}^{\infty}\left(Q_{\Omega} ; \mathbb{R}^{n}\right)$ and $k, l \in\{1, \ldots, n\}$ with $k<l$. Due to $\nabla^{\prime} R_{\varepsilon}=0$ and $\nabla^{\prime} d=0$, one obtains in the case $l<n$ that

$$
\begin{aligned}
& \int_{Q_{\Omega}} U_{\varepsilon} e_{k} \cdot \partial_{l} \varphi \mathrm{d} x-\int_{Q_{\Omega}} U_{\varepsilon} e_{l} \cdot \partial_{k} \varphi \mathrm{d} x \\
& \quad=\int_{Q_{\Omega}} R_{\varepsilon} e_{k} \cdot \partial_{l} \varphi \mathrm{d} x-\int_{Q_{\Omega}} R_{\varepsilon} e_{l} \cdot \partial_{k} \varphi \mathrm{d} x=0
\end{aligned}
$$

and for $l=n$, along with $\partial_{n} R_{\varepsilon}=0$ on $\varepsilon Y_{\text {stiff }} \cap Q_{\Omega}$, that

$$
\begin{aligned}
& \int_{Q_{\Omega}} U_{\varepsilon} e_{k} \cdot \partial_{n} \varphi \mathrm{d} x-\int_{Q_{\Omega}} U_{\varepsilon} e_{n} \cdot \partial_{k} \varphi \mathrm{d} x \\
& =\int_{Q_{\Omega}} R_{\varepsilon} e_{k} \cdot \partial_{n} \varphi \mathrm{d} x-\int_{Q_{\Omega}} \mathbb{1}_{\varepsilon} Y_{\text {soft }}\left(\partial_{n} R_{\varepsilon}\right) x \cdot \partial_{k} \varphi \mathrm{d} x \\
& =\int_{Q_{\Omega}} R_{\varepsilon} e_{k} \cdot \partial_{n} \varphi \mathrm{d} x+\int_{Q_{\Omega}}\left(\partial_{n} R_{\varepsilon}\right) e_{k} \cdot \varphi \mathrm{d} x=0 .
\end{aligned}
$$

Thus, curl $U_{\varepsilon}=0$ as desired.

After restricting $u_{\varepsilon}$ and $R_{\varepsilon}$ to $\Omega$, the statements (4.6) and (4.7) follow now directly from the properties of the sequence $\left(\Sigma_{\varepsilon}\right)_{\varepsilon}$.

The proof of the previous proposition builds builds on a structure preserving approximation result for one-dimensional functions with values in the set of rotations. Let us denote by $I_{\text {soft }}$ the 1-periodic extensions of the interval $(0, \lambda)$ to the real line, which corresponds to a one-dimensional section of $Y_{\text {stiff }}$ in the $e_{n}$-direction, that is $Y_{\text {stiff }}=\mathbb{R}^{n-1} \times I_{\text {stiff }}$. In addition, we set $I_{\text {stiff }}=\mathbb{R}^{n} \backslash I_{\text {soft }}$.

Lemma 4.3. Let $J \subset \mathbb{R}$ be an open and bounded interval, $1 \leqq p<\infty$ and $\Sigma \in W^{1, p}(J ; S O(n))$. Then there exists a sequence $\left(\Sigma_{\varepsilon}\right)_{\varepsilon} \subset \bar{W}^{1, p}(J ; S O(n))$ with

$$
\Sigma_{\varepsilon}^{\prime}=0 \text { a.e. in } \varepsilon I_{\mathrm{stiff}} \cap J
$$

such that $\Sigma_{\varepsilon} \rightarrow \Sigma$ in $W^{1, p}\left(J ; \mathbb{R}^{n \times n}\right)$. Furthermore,

$$
\left\|\Sigma_{\varepsilon}^{\prime}-\frac{1}{\lambda} \Sigma^{\prime}\right\|_{L^{p}\left(\varepsilon I_{\text {soft }} \cap J ; \mathbb{R}^{n \times n}\right)} \rightarrow 0 \text { as } \varepsilon \rightarrow 0 .
$$

Proof. Instead of trying to approximate $\Sigma$ directly with $S O(n)$-valued functions, it seems easier to parametrize $\Sigma$ in a suitable way. Intuitively speaking, the idea is to stop the parametrization on the stiff layers and accelerate it on the softer ones. 


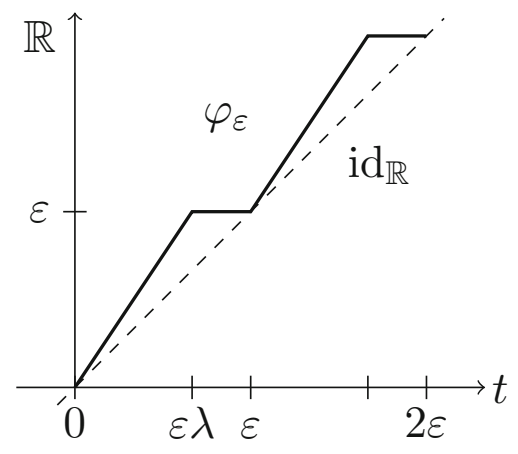

Fig. 8. Illustration of the re-parametrization function $\varphi_{\varepsilon}$

More precisely, for every $\varepsilon>0$, take $\varphi_{\varepsilon}: \mathbb{R} \rightarrow \mathbb{R}$ as the piecewise affine function defined by

$$
\varphi_{\varepsilon}(t)=\varepsilon\lceil t\rceil \text { for } t \in \varepsilon I_{\text {stiff }},
$$

and by linear interpolation on $\varepsilon I_{\text {soft }}$, see Fig. 8 . By construction, one has that

$$
\varphi_{\varepsilon}^{\prime}=\frac{1}{\lambda} \quad \text { on } \varepsilon I_{\text {soft }}
$$

and $\left(\varphi_{\varepsilon}\right)_{\varepsilon}$ converges locally uniformly to the identity function on $\mathbb{R}$ for $\varepsilon \rightarrow 0$.

First, we extend the function $\Sigma$ from $J$ to an open real interval $J^{\prime}$ that contains $J$ compactly. In fact, via reflection one obtains $\Sigma \in W^{1, p}\left(J^{\prime} ; S O(n)\right)$ (not renamed) with

$$
\|\Sigma\|_{W^{1, p}\left(J^{\prime} ; S O(n)\right)} \leqq c\|\Sigma\|_{W^{1, p}(J ; S O(n))}<\infty,
$$

where $c>0$ depends only on $J^{\prime}$.

Next, we define $\Sigma_{\varepsilon}: J \rightarrow S O(n)$ by $\Sigma_{\varepsilon}=\Sigma \circ \varphi_{\varepsilon}$ for sufficiently small $\varepsilon$. Notice that $\Sigma_{\varepsilon}$ is well-defined, since $\varphi_{\varepsilon}(J)=\varphi_{\varepsilon}\left(\varepsilon I_{\text {soft }} \cap J\right) \subset J^{\prime}$ if $\varepsilon$ is small enough. As the composition of an absolutely continuous function with a monotone Lipschitz function, $\Sigma_{\varepsilon}$ is absolutely continuous. In particular, the chain rule holds (see e.g. [44, Theorem 3.44]), i.e.

$$
\Sigma_{\varepsilon}^{\prime}=\left(\Sigma^{\prime} \circ \varphi_{\varepsilon}\right) \varphi_{\varepsilon}^{\prime}
$$

and thus, $\Sigma_{\varepsilon} \in W^{1, p}(J ; S O(n))$. Since $\Sigma_{\varepsilon} \rightarrow \Sigma$ pointwise and the functions $\left|\Sigma_{\varepsilon}\right|^{2} \leqq n$ a.e. in $J$, it follows from Lebesgue's dominated convergence theorem that $\Sigma_{\varepsilon} \rightarrow \Sigma$ in $L^{p}\left(J ; \mathbb{R}^{n \times n}\right)$.

For the asserted weak convergence of $\left(\Sigma_{\varepsilon}\right)_{\varepsilon}$ in $W^{1, p}\left(J ; \mathbb{R}^{n \times n}\right)$, it suffices according to Urysohn's lemma to show that the sequence $\left(\Sigma_{\varepsilon}^{\prime}\right)_{\varepsilon}$ is uniformly bounded in $L^{p}\left(J ; \mathbb{R}^{n \times n}\right)$. Indeed, 


$$
\begin{aligned}
\left\|\Sigma_{\varepsilon}^{\prime}\right\|_{L^{p}\left(J ; \mathbb{R}^{n \times n)}\right.}^{p} & =\int_{\varepsilon I_{\text {soft }} \cap J}\left|\Sigma^{\prime}\left(\varphi_{\varepsilon}\right) \varphi_{\varepsilon}^{\prime}\right|^{2} \mathrm{~d} t=\frac{1}{\lambda^{2}} \int_{\varepsilon I_{\text {soft }} \cap J}\left|\Sigma^{\prime}\left(\varphi_{\varepsilon}\right)\right|^{2} \mathrm{~d} t \\
& =\frac{1}{\lambda^{2}} \sum_{i \in \mathbb{Z}} \int_{\varepsilon(i, i+\lambda) \cap J}\left|\Sigma^{\prime}\left(\varphi_{\varepsilon}\right)\right|^{2} \mathrm{~d} t \\
& \leqq \frac{1}{\lambda^{2}} \sum_{i \in \mathbb{Z}} \int_{\varepsilon(i, i+1) \cap J^{\prime}}\left|\Sigma^{\prime}\right|^{2}\left|\varphi_{\varepsilon}^{\prime}\right|^{-1} \mathrm{~d} t \\
& =\frac{1}{\lambda}\left\|\Sigma^{\prime}\right\|_{L^{2}\left(J^{\prime} ; \mathbb{R}^{n \times n}\right)}^{2}
\end{aligned}
$$

Here we have exploited (4.10) and (4.9), the fact that $\Sigma_{\varepsilon}$ is constant on $\varepsilon I_{\text {stiff }}$, as well as the chain rule and transformation formula on the (finitely many) connected components of $\varepsilon I_{\text {soft }}$, where the restriction of $\varphi_{\varepsilon}$ is invertible.

To show (4.8), we approximate $\Sigma^{\prime}$ in $L^{p}\left(J^{\prime} ; \mathbb{R}^{n \times n}\right)$ by a sequence $\left(g_{j}\right)_{j} \subset$ $C_{c}^{\infty}\left(J^{\prime} ; \mathbb{R}^{n \times n}\right)$. By change of variables on the connected components of $\varepsilon I_{\text {soft }}$ it follows that

$$
\left\|\Sigma^{\prime} \circ \varphi_{\varepsilon}-g_{j} \circ \varphi_{\varepsilon}\right\|_{L^{p}\left(\varepsilon I_{\text {soft }} \cap J ; \mathbb{R}^{n \times n}\right)} \leqq\left\|\Sigma^{\prime}-g_{j}\right\|_{L^{p}\left(J^{\prime} ; \mathbb{R}^{n \times n}\right)},
$$

and therefore

$$
\begin{aligned}
& \left\|\Sigma^{\prime} \circ \varphi_{\varepsilon}-\Sigma^{\prime}\right\|_{L^{p}\left(\varepsilon I_{\text {soft }} \cap J ; \mathbb{R}^{n \times n}\right)} \\
& \leqq\left\|g_{j} \circ \varphi_{\varepsilon}-g_{j}\right\|_{L^{p}\left(J ; \mathbb{R}^{n \times n}\right)}+2\left\|g_{j}-\Sigma^{\prime}\right\|_{L^{p}\left(J^{\prime} ; \mathbb{R}^{n \times n}\right)} \text {. }
\end{aligned}
$$

Since $g_{j} \circ \varphi_{\varepsilon} \rightarrow g_{j}$ in $L^{p}\left(J ; \mathbb{R}^{n \times n}\right)$ for every $j \in \mathbb{N}$ by dominated convergence, passing to the limits $\varepsilon \rightarrow 0$ and $j \rightarrow \infty$ (in this order) in (4.11) proves (4.8).

\section{Homogenization of Layered High-Contrast Materials}

Before proving Theorem 5.2, formulated below, we introduce the setting and precise assumptions. Throughout this section, $\Omega \subset \mathbb{R}^{n}$ is a bounded Lipschitz domain that satisfies the flatness condition and connectedness property of Definitions 3.7 and 3.6, respectively, and $p>n$. For $\varepsilon>0$ and $\alpha>0$ we consider the heterogeneous energy density $W_{\varepsilon}^{\alpha}: \Omega \times \mathbb{R}^{n \times n} \rightarrow[0, \infty)$ given by

$$
W_{\varepsilon}^{\alpha}(x, F)= \begin{cases}\varepsilon^{-\alpha} W_{\text {stiff }}(F) & \text { if } x \in \varepsilon Y_{\text {stiff }} \cap \Omega, \\ W_{\text {soft }}(F) & \text { if } x \in \varepsilon Y_{\text {soft }} \cap \Omega,\end{cases}
$$

where $W_{\text {stiff }}, W_{\text {soft }}: \mathbb{R}^{n \times n} \rightarrow[0, \infty)$ are continuous functions that satisfy the following conditions regarding convexity, growth and coercivity, and local Lipschitz continuity:

(H1) $W_{\text {soft }}^{\mathrm{qc}}$ is polyconvex;

(H2) $c|F|^{p}-\frac{1}{C} \leqq W_{\text {soft }}(F) \leqq C\left(1+|F|^{p}\right)$ for all $F \in \mathbb{R}^{n \times n}$ with constants $C, c>0$

(H3) $\left|W_{\text {soft }}(F)-W_{\text {soft }}(G)\right| \leqq L\left(1+|F|^{p-1}+|G|^{p-1}\right)|F-G|$ for all $F, G \in$ $\mathbb{R}^{n \times n}$ with $L>0$; 
(H4) $W_{\text {stiff }}(F) \geqq k \operatorname{dist}^{p}(F, S O(n))$ for all $F \in \mathbb{R}^{n \times n}$ with a constant $k>0$.

An equivalent way of expressing $(H 1)$ is by

$$
W_{\mathrm{soft}}^{\mathrm{qc}}=W_{\mathrm{soft}}^{\mathrm{pc}}
$$

where $W_{\text {soft }}^{\mathrm{qc}}$ and $W_{\text {soft }}^{\mathrm{pc}}$ are the quasiconvex and polyconvex envelopes of $W_{\text {soft }}$, that is, the largest quasiconvex and polyconvex functions below $W_{\text {soft }}$. For a detailed introduction to generalized notions of convexity and the corresponding generalized convexifications we refer to [25]. Let us just recall briefly that a continuous function $W: \mathbb{R}^{n \times n} \rightarrow \mathbb{R}$ with standard $p$-growth (i.e., with an the upper bound as in (H2)) is quasiconvex if for any $F \in \mathbb{R}^{n \times n}$,

$$
\inf _{\varphi \in W_{0}^{1, p}\left((0,1)^{n} ; \mathbb{R}^{n}\right)} f_{(0,1)^{n}} W(F+\nabla \varphi) \mathrm{d} x \geqq W(F) .
$$

Moreover, a continuous $W: \mathbb{R}^{n \times n} \rightarrow \mathbb{R}$ is polyconvex if there exists a convex function $g: \mathbb{R}^{\tau(n)} \rightarrow \mathbb{R}$ such that

$$
W(F)=g(\mathcal{M}(F)) \text { for all } F \in \mathbb{R}^{n \times n},
$$

where $\mathcal{M}(F) \in \mathbb{R}^{\tau(n)}$ with $\tau(n)=\sum_{i=1}^{n}\left(\begin{array}{l}n \\ k\end{array}\right)$ is the vector of minors of $F$.

We remark that explicit formulas for quasiconvex envelopes are in general hard to obtain. This is why quasiconvexifications are rather rare in the literature, see e.g. [21,24,43] for a few examples (including extended-valued densities). A common strategy is to determine upper and lower bounds in terms of rank-one and polyconvex envelopes and to show that the latter two match. Hence, in those cases where relaxations are explicitly known, $(H 1)$ is usually satisfied.

Example 5.1. Let $n=2$ or $n=3$. The Saint Venant-Kirchhoff stored energy function,

$$
W_{S K}(F)=\frac{\lambda}{4}\left|F^{T} F-\mathbb{I}\right|^{2}+\frac{\mu}{8}\left(|F|^{2}-n\right)^{2}, \quad F \in \mathbb{R}^{n \times n},
$$

with the Lamé constants $\lambda, \mu>0$, is one of the simplest energy densities of relevance in hyperelasticity (see e.g. [36, Section 28]), and meets requirements for $W_{\text {soft }}$. It is straightforward to see that $W_{S K}$ has standard growth $(H 2)$ with $p=4$ and is locally Lipschitz continuous in the sense of (H3). In [43], Le Dret and Raoult give an explicit expression of the quasiconvexification $W_{S K}^{\mathrm{qc}}$, which coincides with the convex, polyconvex and rank-one convex envelopes. Thus, in particular, $(H 1)$ is satisfied, too.

Let $E_{\varepsilon}: L^{p}\left(\Omega ; \mathbb{R}^{n}\right) \rightarrow \mathbb{R} \cup\{\infty\}$ be the integral functional with density $W_{\varepsilon}^{\alpha}$, i.e.

$$
E_{\varepsilon}(u)=\int_{\Omega} W_{\varepsilon}^{\alpha}(x, \nabla u) \mathrm{d} x
$$

if $u \in W^{1, p}\left(\Omega ; \mathbb{R}^{n}\right)$ and $E_{\varepsilon}(u)=\infty$ otherwise in $L^{p}\left(\Omega ; \mathbb{R}^{n}\right)$. 
Recalling that $\mathcal{A}=\left\{F \in \mathbb{R}^{n \times n}: F=R_{F}+d_{F} \otimes e_{n}\right.$ with $R_{F} \in S O(n)$ and $d_{F} \in$ $\left.\mathbb{R}^{n}\right\}$ (cf. (4.2)), we define for $F \in \mathcal{A}$,

$$
W_{\text {hom }}(F)=\lambda W_{\text {soft }}^{\mathrm{qc}}\left(F_{\lambda}\right)=\lambda \inf _{\varphi \in W_{0}^{1, p}\left((0,1)^{n} ; \mathbb{R}^{n}\right)} f_{(0,1)^{n}} W_{\text {soft }}\left(F_{\lambda}+\nabla \varphi\right) \mathrm{d} x,
$$

where $F_{\lambda}=R_{F}+\frac{1}{\lambda} d_{F} \otimes e_{n}=\frac{1}{\lambda}\left(F-(1-\lambda) R_{F}\right) \in \mathcal{A}$.

Now we are ready to formulate the main theorem of this section. Theorem 5.2 provides a characterization of the effective behavior of the bilayered materials modeled by (5.3) by homogenization via $\Gamma$-convergence for vanishing layer thickness. The limit problem shows a splitting of the effects of the heterogeneities and relaxation of microstructures on the softer components. With regards to homogenization, the resulting formulas are explicit and can be expressed in terms of the relative layer thickness. Provided the relaxation of $W_{\text {soft }}$ is known, $W_{\text {hom }}$ is even fully explicit.

Theorem 5.2. If $\alpha>p$, the family $\left(E_{\varepsilon}\right)_{\varepsilon}$ as in (5.3) converges in the sense of $\Gamma$-convergence regarding the strong $L^{p}$-topology to the limit functional $E_{\mathrm{hom}}$ : $L^{p}\left(\Omega ; \mathbb{R}^{n}\right) \rightarrow \mathbb{R}_{\infty}$ given by

$E_{\mathrm{hom}}(u)= \begin{cases}\int_{\Omega} W_{\mathrm{hom}}(\nabla u) \mathrm{d} x & \text { if } u(x)=R(x) x+b(x) \text { with } R \in W^{1, p}(\Omega ; S O(n)) \text { such } \\ & \text { that } \nabla^{\prime} R=0 \text { and } b \in W^{1, p}\left(\Omega ; \mathbb{R}^{n}\right) \text { such that } \nabla^{\prime} b=0, \\ & \text { otherwise. }\end{cases}$

Precisely, this means that the following two conditions are satisfied:

(i) (Lower bound) For each $u \in L^{p}\left(\Omega ; \mathbb{R}^{n}\right)$ and any sequence $\left(u_{\varepsilon}\right)_{\varepsilon} \subset L^{p}\left(\Omega ; \mathbb{R}^{n}\right)$ with $u_{\varepsilon} \rightarrow u$ in $L^{p}\left(\Omega ; \mathbb{R}^{n}\right)$ as $\varepsilon \rightarrow 0$ it holds that

$$
\liminf _{\varepsilon \rightarrow 0} E_{\varepsilon}\left(u_{\varepsilon}\right) \geqq E_{\mathrm{hom}}(u) ;
$$

(ii) (Existence of a recovery sequence) For each $u \in L^{p}\left(\Omega ; \mathbb{R}^{n}\right)$ there exists a sequence $\left(u_{\varepsilon}\right)_{\varepsilon} \subset L^{p}\left(\Omega ; \mathbb{R}^{n}\right)$ with $u_{\varepsilon} \rightarrow u$ in $L^{p}\left(\Omega ; \mathbb{R}^{n}\right)$ as $\varepsilon \rightarrow 0$ such that

$$
\lim _{\varepsilon \rightarrow 0} E_{\varepsilon}\left(u_{\varepsilon}\right)=E_{\mathrm{hom}}(u) .
$$

Moreover, any sequence $\left(u_{\varepsilon}\right)_{\varepsilon} \subset L_{0}^{p}\left(\Omega ; \mathbb{R}^{n}\right)$ of uniformly bounded energy for $\left(E_{\varepsilon}\right)_{\varepsilon}$, that is $E_{\varepsilon}\left(u_{\varepsilon}\right)<C$ for all $\varepsilon>0$, is relatively compact in $L^{p}\left(\Omega ; \mathbb{R}^{n}\right)$.

Remark 5.3. (a) If $W_{\text {soft }}$ is convex, then $W_{\text {soft }}^{\mathrm{qc}}=W_{\text {soft }}^{\mathrm{c}}=W_{\text {soft }}$, so that $W_{\text {hom }}(F)=$ $\lambda W_{\text {soft }}\left(F_{\lambda}\right)$ for $F \in \mathcal{A}$. In this case, the proof of Theorem 5.2 can be simplified as indicated below.

(b) It is well-known that the definition of quasiconvexity in (5.2), as well as the representation formula for the quasiconvex envelope $W^{\mathrm{qc}}$, is independent of the choice of the domain, see e.g. [25, Proposition 5.11]. Therefore, we have for any open set $O \subset \mathbb{R}^{n}$ that

$$
W_{\text {soft }}^{\mathrm{qc}}(F)=\inf _{\varphi \in W_{0}^{1, p}\left(O ; \mathbb{R}^{n}\right)} f_{O} W_{\text {soft }}(F+\nabla \varphi) \mathrm{d} y, \quad F \in \mathbb{R}^{n \times n} .
$$


Alternatively, $W_{\text {soft }}^{\mathrm{qc}}$ can be expressed with the help periodic perturbations on a cube $Q \subset \mathbb{R}^{n}$ as

$$
W_{\text {soft }}^{\mathrm{qc}}(F)=\inf _{\varphi \in W_{\#}^{1, p}\left(Q ; \mathbb{R}^{n}\right)} f_{Q} W_{\text {soft }}(F+\nabla \varphi) \mathrm{d} y, \quad F \in \mathbb{R}^{n \times n},
$$

see e.g. [49, Proposition 4.19] or [25, Proposition 5.13].

(c) The homogenized energy density $W_{\text {hom }}$ is non-negative and inherits the property $(H 2)$ from $W_{\text {soft }}$. This follows from the fact that $W_{\text {soft }}^{\mathrm{qc}}$ has standard $p$ growth, because $W_{\text {soft }}$ has, along with the estimate

$$
\frac{1}{2 \lambda}|F|-\frac{n}{\lambda} \leqq\left|F_{\lambda}\right| \leqq \frac{1}{\lambda}(|F|+1) \text { for } F \in \mathcal{A} .
$$

Moreover, $W_{\text {hom }}$ is locally Lipschitz continuous in the sense that, just as $W_{\text {hom }}$, it satisfies hypothesis (H3). Precisely, one can find $L_{\text {hom }}>0$ such that

$\left|W_{\text {hom }}(F)-W_{\text {hom }}(G)\right| \leqq L_{\text {hom }}\left(1+|F|^{p-1}+|G|^{p-1}\right)|F-G|$ for all $F, G \in \mathcal{A}$.

To see this, we exploit that the property $(H 3)$ carries over from $W_{\text {soft }}$ to $W_{\text {soft }}^{\mathrm{qc}}$ (cf. e.g. [48, Lemma 2.1 c)]). Hence,

$$
\begin{aligned}
& \left|W_{\text {hom }}(F)-W_{\text {hom }}(G)\right| \leqq \lambda\left|W_{\text {soft }}^{\mathrm{qc}}\left(F_{\lambda}\right)-W_{\text {soft }}^{\mathrm{qc}}\left(G_{\lambda}\right)\right| \\
& \quad \leqq \lambda \tilde{L}\left(1+\left|F_{\lambda}\right|^{p-1}+\left|G_{\lambda}\right|^{p-1}\right)\left|F_{\lambda}-G_{\lambda}\right|
\end{aligned}
$$

for $F, G \in \mathcal{A}$ with a constant $\tilde{L}>0$. In view of (5.5), it only remains to estimate $\left|F_{\lambda}-G_{\lambda}\right|$ suitably from above by $|F-G|$. We observe that

$$
\begin{aligned}
\left|F_{\lambda}-G_{\lambda}\right| & \leqq \frac{1}{\lambda}|F-G|+\frac{1-\lambda}{\lambda}\left(|\widehat{F}-\widehat{G}|+\left|R_{F} e_{n}-R_{G} e_{n}\right|\right) \\
& \leqq \frac{2-\lambda}{\lambda}|F-G|+\frac{1-\lambda}{\lambda}\left|R_{F} e_{n}-R_{G} e_{n}\right|,
\end{aligned}
$$

where $\widehat{A}$ stands for the $n \times(n-1)$-matrix that results from removing the last column of $A \in \mathbb{R}^{n \times n}$. We denote the $n$-dimensional cross product of vectors $v_{1}, \ldots, v_{n} \in \mathbb{R}^{n}$ by $v_{1} \times \cdots \times v_{n-1}=\times_{i=1}^{n-1} v_{i} \in \mathbb{R}^{n}$. The latter is by definition the uniquely determined vector that is orthogonal on the hyperplane spanned by $v_{1}, \ldots, v_{n-1}$ such that the orientation of $v_{1}, \ldots, v_{n-1}, \times_{i=1}^{n-1} v_{i}$ is positive and its norm is the volume of the parallelotope associated with $v_{1}, \ldots, v_{n-1}$. For every rotation $R \in S O(n)$, one has that $R e_{n}=\times_{i=1}^{n-1} R e_{i}$.

The multilinearity of the cross product in $\mathbb{R}^{n}$ and the fact that $\left|R_{F} e_{i}\right|=$ $\left|R_{G} e_{i}\right|=1$ for $i=1, \ldots, n$ allows us to obtain iteratively that

$$
\begin{aligned}
\left|R_{F} e_{n}-R_{G} e_{n}\right|= & \left|\times_{i=1}^{n-1} R_{F} e_{i}-\times_{i=1}^{n-1} R_{G} e_{i}\right| \\
\leqq & \left|R_{F} e_{1}-R_{G} e_{1}\right|+\mid R_{G} e_{1} \times R_{G} e_{2} \times \cdots \times R_{G} e_{n-1}-R_{G} e_{1} \\
& \times R_{F} e_{2} \times \cdots \times R_{F} e_{n-1} \mid \\
\leqq & \cdots \leqq \sum_{i=1}^{n-1}\left|R_{F} e_{i}-R_{G} e_{i}\right| \leqq(n-1)\left|\widehat{R_{F}}-\widehat{R_{G}}\right| \leqq(n-1)|F-G| .
\end{aligned}
$$

Finally, we combine the above estimates to deduce the desired local Lipschitz property (5.6). 
(d) As mentioned in the introduction, proving a $\Gamma$-limit homogenization result as above without the hypothesis $(H 1)$ is an open problem. In any case, Theorem 5.2 provides an upper bound on the $\Gamma$-limit (if existent) in that situation.

We subdivide the proof of Theorem 5.2 into three main parts. After showing compactness, we first determine the homogenization $\Gamma$-limit for all affine functions, and then prove the general statement via a localization argument. Note that the specific structure of the admissible limit deformations as characterized in Theorem 3.1, in particular the resulting multiplicative separation of $x^{\prime}$ and $x_{n}$-variables in (4.1), is key. This observation allows us to construct an approximation that fulfills the (asymptotic) constraints on the stiff layers, cf. Proposition 4.1.

The first part of the proof is standard, yet, we sketch it here for the readers' convenience.

Proof of Theorem 5.2 (Part I): Compactness. Let $\left(u_{\varepsilon}\right)_{\varepsilon} \subset L_{0}^{p}\left(\Omega ; \mathbb{R}^{n}\right)$ be such that $E_{\varepsilon}\left(u_{\varepsilon}\right)<C$ for all $\varepsilon>0$. Then, $\operatorname{since} \operatorname{dist}(F, S O(n)) \geqq|F|-\sqrt{n}$ for all $F \in \mathbb{R}^{n \times n}$, the lower bounds on $W_{\text {soft }}$ and $W_{\text {stiff }}$ in (H2) and (H4), imply that $\left(\nabla u_{\varepsilon}\right)_{\varepsilon}$ is uniformly bounded in $L^{p}\left(\Omega ; \mathbb{R}^{n}\right)$. The stated relative compactness of $\left(u_{\varepsilon}\right)_{\varepsilon}$ in $L^{p}\left(\Omega ; \mathbb{R}^{n}\right)$ follows now from Poincaré's inequality, which shows that

$$
\left\|u_{\varepsilon}\right\|_{W^{1, p}\left(\Omega ; \mathbb{R}^{n}\right)} \leqq C \text { for all } \varepsilon>0,
$$

along with the compact embedding $W^{1, p}\left(\Omega ; \mathbb{R}^{n}\right) \hookrightarrow \hookrightarrow L^{p}\left(\Omega ; \mathbb{R}^{n}\right)$.

Proof of Theorem 5.2 (Part II): Affine case. Suppose that $u \in W^{1, p}\left(\Omega ; \mathbb{R}^{n}\right)$ with $E_{\text {hom }}(u)<\infty$ is affine. Hence, there is $F \in \mathbb{R}^{n \times n}$ with $F \in \mathcal{A}$, cf. (4.2).

Step 1: Existence of a recovery sequence The construction of a recovery sequence for $u$ as above, that is, finding $\left(u_{\varepsilon}\right)_{\varepsilon} \subset L^{p}\left(\Omega ; \mathbb{R}^{n}\right)$ with

$$
u_{\varepsilon} \rightarrow u \text { in } L^{p}\left(\Omega ; \mathbb{R}^{m}\right) \text { and } E_{\varepsilon}\left(u_{\varepsilon}\right) \rightarrow E_{\mathrm{hom}}(u) \text { as } \varepsilon \rightarrow 0
$$

requires a careful adaptation of by now classical techniques, see e.g. [47]. Indeed, instead of glueing small-scale oscillations on top of an affine function, the former are glued onto an appropriate laminate, namely the one constructed in (4.4).

Let $\delta>0$. In view of Remark $5.3 \mathrm{~b})$, one can find $\varphi_{\delta} \in W_{0}^{1, p}\left(Y_{\mathrm{soft}} ; \mathbb{R}^{n}\right)$ such that

$$
W_{\text {soft }}^{\mathrm{qc}}\left(F_{\lambda}\right) \leqq f_{Y_{\text {soft }}} W_{\text {soft }}\left(F_{\lambda}+\nabla \varphi_{\delta}\right) \mathrm{d} y \leqq W_{\text {soft }}^{\mathrm{qc}}\left(F_{\lambda}\right)+\delta .
$$

We set $\varphi_{\delta}$ equal to zero in the remainder of the unit cube and extend it $Y$-periodically to $\mathbb{R}^{n}$. For $\varepsilon>0$ let $v_{\varepsilon}^{F}$ be a Lipschitz function with gradients as in (4.4) and vanishing mean value on $\Omega$. Then, $v_{\varepsilon}^{F} \rightarrow u$ in $L^{p}\left(\Omega ; \mathbb{R}^{n}\right)$ as $\varepsilon \rightarrow 0$. With

$$
u_{\delta, \varepsilon}(x)=v_{\varepsilon}^{F}(x)+\varepsilon \varphi_{\delta}\left(\frac{x}{\varepsilon}\right), \quad x \in \Omega,
$$

it follows that $u_{\delta, \varepsilon} \rightarrow u$ in $L^{p}\left(\Omega ; \mathbb{R}^{n}\right)$ as $\varepsilon \rightarrow 0$. Regarding energies, we obtain that

$$
\begin{aligned}
E_{\varepsilon}\left(u_{\delta, \varepsilon}\right) & =\int_{\varepsilon Y_{\text {soft }} \cap \Omega} W_{\text {soft }}\left(F_{\lambda}+\nabla \varphi_{\delta}\left(\frac{x}{\varepsilon}\right)\right) \mathrm{d} x \\
& =\int_{\Omega} W_{\text {soft }}\left(F_{\lambda}+\nabla \varphi_{\delta}\left(\frac{x}{\varepsilon}\right)\right) \mathbb{1}_{Y_{\text {soft }}}\left(\frac{x}{\varepsilon}\right) \mathrm{d} x .
\end{aligned}
$$


Hence, as $\varepsilon$ tends to zero,

$$
\begin{aligned}
\lim _{\varepsilon \rightarrow 0} E_{\varepsilon}\left(u_{\delta, \varepsilon}\right) & =|\Omega| \int_{Y} W_{\text {soft }}\left(F_{\lambda}+\nabla \varphi_{\delta}\right) \mathbb{1}_{Y_{\text {soft }}} \mathrm{d} x \\
& =\lambda|\Omega| \int_{Y_{\text {soft }}} W_{\text {soft }}\left(F_{\lambda}+\nabla \varphi_{\delta}\right) \mathrm{d} x,
\end{aligned}
$$

and we infer, along with (5.8), that

$$
E_{\mathrm{hom}}\left(u_{\delta}\right) \leqq \lim _{\varepsilon \rightarrow 0} E_{\varepsilon}\left(u_{\delta, \varepsilon}\right) \leqq E_{\mathrm{hom}}\left(u_{\delta}\right)+\lambda|\Omega| \delta .
$$

By Attouch's diagonalization lemma (see e.g. [4, Lemma 1.15, Corollary 1.16]) there exist $\delta(\varepsilon)$ such that $u_{\delta(\varepsilon), \varepsilon} \rightarrow u$ in $L^{p}\left(\Omega ; \mathbb{R}^{n}\right)$ and $E_{\varepsilon}\left(u_{\delta(\varepsilon), \varepsilon}\right) \rightarrow E_{\text {hom }}(u)$ as $\varepsilon \rightarrow 0$. Finally, defining $u_{\varepsilon}:=u_{\delta(\varepsilon), \varepsilon}$ yields the desired recovery sequence for $u$.

Step 2: Lower bound Let $\left(u_{\varepsilon}\right)_{\varepsilon} \subset L^{p}\left(\Omega ; \mathbb{R}^{n}\right)$ be such that $u_{\varepsilon} \rightarrow u$ in $L^{p}\left(\Omega ; \mathbb{R}^{n}\right)$. Since the energies $E_{\varepsilon}$ and $E_{\text {hom }}$ depend only on gradients, one may assume that the functions $u_{\varepsilon}$ and $u$ have vanishing mean value, i.e., $u_{\varepsilon}, u \in L_{0}^{p}\left(\Omega ; \mathbb{R}^{n}\right)$. We will show that

$$
\liminf _{\varepsilon \rightarrow 0} E_{\varepsilon}\left(u_{\varepsilon}\right) \geqq E_{\mathrm{hom}}(u) .
$$

Without loss of generality, let $\liminf _{\varepsilon \rightarrow 0} E_{\varepsilon}\left(u_{\varepsilon}\right)=\lim _{\varepsilon \rightarrow 0} E_{\varepsilon}\left(u_{\varepsilon}\right)<\infty$. In view of Part I, one may further assume that

$$
u_{\varepsilon} \rightarrow u \quad \text { in } W^{1, p}\left(\Omega ; \mathbb{R}^{n}\right)
$$

We remark that (5.9) follows immediately, if one can prove that

$$
\liminf _{\varepsilon \rightarrow 0} \int_{\varepsilon Y_{\text {soft }} \cap Q} W_{\text {soft }}\left(\nabla u_{\varepsilon}\right) \mathrm{d} x \geqq \int_{Q} W_{\text {hom }}(\nabla u) \mathrm{d} x=|Q| W_{\text {hom }}(F)
$$

for any open cuboid $Q=O \times J \subset \subset \Omega$, where $O \subset \mathbb{R}^{n-1}$ and $J \subset \mathbb{R}$ and open interval. To deduce (5.9), we can then exhaust $\Omega$ with disjoint cuboids $Q_{i} \subset \Omega$ for $i \in \mathbb{N}$ such that $\left|\Omega \backslash \bigcup_{i=1}^{\infty} Q_{i}\right|=0$ and apply (5.9) on each $Q_{i}$. More precisely, for any $N \in \mathbb{N}$,

$$
\liminf _{\varepsilon \rightarrow 0} \int_{\Omega} W_{\varepsilon}^{\alpha}\left(\nabla u_{\varepsilon}\right) \mathrm{d} x \geqq \sum_{i=1}^{N} \int_{\varepsilon Y_{\text {soft }} \cap Q_{i}} W_{\text {soft }}\left(\nabla u_{\varepsilon}\right) \mathrm{d} x \geqq\left|\bigcup_{i=1}^{N} Q_{i}\right| W_{\text {hom }}(F),
$$

so that taking the supremum over $N \in \mathbb{N}$ implies (5.9).

It remains to prove (5.11), which relies substantially on hypothesis $(H 1)$, or equivalently on (5.1). Since $W_{\text {soft }}^{\mathrm{pc}}$ is polyconvex, we can find a convex function $g: \mathbb{R}^{\tau(n)} \rightarrow \mathbb{R}$ such that $W^{\mathrm{pc}}(F)=g(\mathcal{M}(F))$ for all $F \in \mathbb{R}^{n \times n}$. Moreover, let $P_{\varepsilon}^{i}=\left(\mathbb{R}^{n-1} \times \varepsilon[i, i+1)\right) \cap Q$ for $i \in \mathbb{N}$ and $I_{\varepsilon} \subset \mathbb{N}$ an index set such that $i \in I_{\varepsilon}$ if and only if $\left|P_{\varepsilon}^{i}\right|=\varepsilon|O|$. As a consequence, $\left|\varepsilon Y_{\text {soft }} \cap P_{\varepsilon}^{i}\right|=\lambda \varepsilon|O|$ for all $i \in I_{\varepsilon}$, and one finds with $Q_{\varepsilon}=\bigcup_{i \in I_{\varepsilon}} P_{\varepsilon}^{i} \subset Q$ that

$$
\left|Q_{\varepsilon}\right|=\varepsilon \# I_{\varepsilon} \rightarrow|Q| \text { and }\left|\varepsilon Y_{\text {soft }} \cap Q_{\varepsilon}\right| \rightarrow \lambda|Q| \quad \text { as } \varepsilon \rightarrow 0 \text {. }
$$


Due to the convexity of $g$ we can invoke Jensen's inequality, applied twice, first in the version for Lebesgue-measurable functions and second in the discrete version, to obtain

$$
\begin{aligned}
\int_{\varepsilon Y_{\text {soft }} \cap Q} W_{\text {soft }}\left(\nabla u_{\varepsilon}\right) \mathrm{d} x & \geqq \int_{\varepsilon Y_{\text {soft }} \cap Q} W_{\text {soft }}^{\mathrm{pc}}\left(\nabla u_{\varepsilon}\right) \mathrm{d} x \geqq \sum_{i \in I_{\varepsilon}} \int_{\varepsilon Y_{\text {soft }} \cap P_{\varepsilon}^{i}} g\left(\mathcal{M}\left(\nabla u_{\varepsilon}\right)\right) \mathrm{d} x \\
& \geqq \lambda \varepsilon \sum_{i \in I_{\varepsilon}} g\left(f_{\varepsilon Y_{\text {soft }} \cap P_{\varepsilon}^{i}} \mathcal{M}\left(\nabla u_{\varepsilon}\right) \mathrm{d} x\right) \\
& \geqq \lambda \varepsilon \# I_{\varepsilon} g\left(\frac{1}{\# I_{\varepsilon}} \sum_{i \in I_{\varepsilon}} f_{\varepsilon Y_{\text {soft }} \cap P_{\varepsilon}^{i}} \mathcal{M}\left(\nabla u_{\varepsilon}\right) \mathrm{d} x\right) \\
& =\lambda\left|Q_{\varepsilon}\right| g\left(f_{\varepsilon Y_{\text {soft }} \cap Q_{\varepsilon}} \mathcal{M}\left(\nabla u_{\varepsilon}\right) \mathrm{d} x\right) .
\end{aligned}
$$

With the aim of eventually passing to the limit $\varepsilon \rightarrow 0$ in (5.13), we will show first that

$$
\mathcal{M}\left(\nabla u_{\varepsilon}\right) \mathbb{1}_{\varepsilon} Y_{\text {soft }} \cap Q \rightarrow \mathcal{M}(F)-(1-\lambda) \mathcal{M}\left(R_{F}\right) \text { in } L^{1}\left(Q ; \mathbb{R}^{\tau(n)}\right) .
$$

For this the properties of $u_{\varepsilon}$ due to the presence of the stiff layers need to be taken into account. Owing to $(H 4)$ and (5.10), the sequence $\left(u_{\varepsilon}\right)_{\varepsilon}$ satisfies the requirements of Theorem 3.1, and also Corollary 3.8. Following the proofs, we find the onedimensional auxiliary sequence $\left(\Sigma_{\varepsilon}\right)_{\varepsilon} \subset L^{p}(J ; S O(n))$ defined in (3.10) with the properties that $\Sigma_{\varepsilon} \rightarrow \Sigma_{0}$ in $L^{p}\left(J ; \mathbb{R}^{n \times n}\right)$ and $\Sigma_{0}\left(x_{n}\right)=R_{F}$ for $x \in Q$, cf. (3.15) and (3.18).

For each $\varepsilon$, we extend $\Sigma_{\varepsilon}$ constantly in $x^{\prime}$ and call the resulting function $S_{\varepsilon} \in$ $L^{\infty}(Q ; S O(n))$. As a consequence of (3.6) (cf. also (3.10)) it holds that

$$
\left\|\nabla u_{\varepsilon}-S_{\varepsilon}\right\|_{L^{p}\left(\varepsilon Y_{\text {stiff }} \cap Q ; \mathbb{R}^{n \times n}\right)} \leqq C \varepsilon^{\frac{\alpha}{p}-1} .
$$

Summing up, we have hence found a sequence $\left(S_{\varepsilon}\right)_{\varepsilon} \subset L^{\infty}(Q ; S O(n))$ such that

$$
S_{\varepsilon} \rightarrow R_{F} \quad \text { in } L^{p}\left(Q ; \mathbb{R}^{n \times n}\right) \text { and }\left\|\nabla u_{\varepsilon}-S_{\varepsilon}\right\|_{L^{p}\left(\varepsilon Y_{\text {stiff }} \cap Q ; \mathbb{R}^{n \times n}\right)} \rightarrow 0
$$

as $\varepsilon \rightarrow 0$.

To see (5.14), let us rewrite the expression $\mathcal{M}\left(\nabla u_{\varepsilon}\right) \mathbb{1}_{\varepsilon Y_{\text {soft }} \cap Q}$ as follows:

$$
\begin{aligned}
\mathcal{M}\left(\nabla u_{\varepsilon}\right) \mathbb{1}_{\varepsilon} Y_{\text {soft }} \cap Q & =\mathcal{M}\left(\nabla u_{\varepsilon}\right)-\mathcal{M}\left(\nabla u_{\varepsilon}\right) \mathbb{1}_{\varepsilon} Y_{\text {stiff }} \cap Q \\
& =\mathcal{M}\left(\nabla u_{\varepsilon}\right)-\left(\mathcal{M}\left(\nabla u_{\varepsilon}\right)-\mathcal{M}\left(S_{\varepsilon}\right)\right) \mathbb{1}_{\varepsilon} Y_{\text {stiff }} \cap Q-\mathcal{M}\left(S_{\varepsilon}\right) \mathbb{1}_{\varepsilon} Y_{\text {stiff }} \cap Q
\end{aligned}
$$

It is well-known that for $p>n$ weak continuity of minors holds, that is, $\mathcal{M}\left(\nabla u_{\varepsilon}\right) \rightarrow$ $\mathcal{M}(\nabla u)=\mathcal{M}(F)$ in $L^{1}\left(\Omega ; \mathbb{R}^{\tau(n)}\right)$, see e.g. [25, Theorem 8.20, Part 4]. Furthermore, the first convergence in $(5.15)$ yields

$$
\mathcal{M}\left(S_{\varepsilon}\right) \rightarrow \mathcal{M}\left(R_{F}\right) \quad \text { in } L^{1}\left(Q ; \mathbb{R}^{\tau(n)}\right),
$$


while the second allows us to conclude that

$$
\left\|\mathcal{M}\left(\nabla u_{\varepsilon}\right)-\mathcal{M}\left(S_{\varepsilon}\right)\right\|_{L^{1}\left(\varepsilon Y_{\text {stiff }} \cap Q ; \mathbb{R}^{\tau(n)}\right)} \rightarrow 0 .
$$

Both convergences follow from the following estimate based on the Leibniz formula for determinants, together with Hölder's inequality and the uniform bounds on $\left(S_{\varepsilon}\right)_{\varepsilon}$ and $\left(\nabla u_{\varepsilon}\right)_{\varepsilon}$ in $L^{\infty}\left(\Omega ; \mathbb{R}^{n \times n}\right)$ and $L^{p}\left(\Omega ; \mathbb{R}^{n \times n}\right)$, respectively. For any $A, B \in$ $\mathbb{R}^{m \times m}$,

$$
\begin{aligned}
|\operatorname{det} A-\operatorname{det} B| & =\left|\sum_{\sigma \in \mathcal{S}_{m}} \operatorname{sgn}(\sigma)\left(\prod_{i=1}^{m} A_{i, \sigma(i)}-\prod_{i=1}^{m} B_{i, \sigma(i)}\right)\right| \\
& \leqq \sum_{\sigma \in \mathcal{S}_{m}} \sum_{j=1}^{m}\left|\left(A_{j, \sigma(j)}-B_{j, \sigma(j)}\right) \cdot \prod_{1 \leqq k<j} A_{k, \sigma(k)} \cdot \prod_{j<\ell \leqq m} B_{\ell, \sigma(\ell)}\right|
\end{aligned}
$$

where $\mathcal{S}_{m}$ denotes the symmetric group over a set of $m$ elements. To give more detail regarding the argument behind (5.17), we observe that with $\mathcal{M}^{m}(F)$ denoting any subdeterminant of $F \in \mathbb{R}^{n \times n}$ of order $m \leqq n$,

$$
\begin{aligned}
& \left\|\mathcal{M}^{m}\left(\nabla u_{\varepsilon}\right)-\mathcal{M}^{m}\left(S_{\varepsilon}\right)\right\|_{L^{1}\left(\varepsilon Y_{\text {stiff }} \cap Q\right)} \leqq|Q|^{\frac{p-m}{p}}\left\|\mathcal{M}^{m}\left(\nabla u_{\varepsilon}\right)-\mathcal{M}^{m}\left(S_{\varepsilon}\right)\right\|_{L^{\frac{p}{m}}\left(\varepsilon Y_{\text {stiff }} \cap Q\right)} \\
& \quad \leqq|Q|^{\frac{p-m}{p}} \sum_{\sigma \in \mathcal{S}_{m}} \sum_{j=1}^{m}\left\|\nabla u_{\varepsilon}\right\|_{L^{p}\left(Q ; \mathbb{R}^{n \times n}\right)}^{j-1}\left\|S_{\varepsilon}\right\|_{L^{p}\left(Q ; \mathbb{R}^{n \times n}\right)}^{m-j}\left\|\left(\nabla u_{\varepsilon}\right)_{j, \sigma(j)}-\left(S_{\varepsilon}\right)_{j, \sigma(j)}\right\|_{L^{p}\left(\varepsilon Y_{\text {stiff }} \cap Q\right)} \\
& \quad \leqq C\left\|\nabla u_{\varepsilon}-S_{\varepsilon}\right\|_{L^{p}\left(\varepsilon Y_{\text {stiff }} \cap Q ; \mathbb{R}^{n \times n}\right)},
\end{aligned}
$$

with a constant $C>0$ depending on $m, p$ and $Q$ and the uniform bound on $\left\|\nabla u_{\varepsilon}\right\|_{L^{p}\left(\Omega ; \mathbb{R}^{n \times n}\right)}$.

From the lemma on weak convergence of highly oscillating periodic functions [18, Section 2.3] we infer that $\mathbb{1}_{\varepsilon} Y_{\text {stiff }} \cap Q \stackrel{*}{\rightarrow}(1-\lambda)$ in $L^{\infty}(Q)$. Finally, applying these results to the individual terms in (5.16) along with a weak-strong convergence argument implies (5.14).

Next, we observe that, as a Null-Lagrangian or polyaffine function, $G \mapsto$ $\mathcal{M}(G)$ for $G \in \mathbb{R}^{n \times n}$ is also rank-one affine, cf. [25, Theorem 5.20]. Since $F=\lambda F_{\lambda}+(1-\lambda) R_{F}$ and $F_{\lambda}-R_{F}=\frac{1}{\lambda}\left(F-R_{F}\right)=\frac{1}{\lambda} d_{F} \otimes e_{n}$, it follows that

$$
\mathcal{M}(F)=\lambda \mathcal{M}\left(F_{\lambda}\right)+(1-\lambda) \mathcal{M}\left(R_{F}\right) .
$$

Then, together with (5.14), we obtain

$$
\mathcal{M}\left(\nabla u_{\varepsilon}\right) \mathbb{1}_{\varepsilon Y_{\text {soft }} \cap Q} \rightarrow \lambda \mathcal{M}\left(F_{\lambda}\right) \text { in } L^{1}\left(Q ; \mathbb{R}^{\tau(n)}\right),
$$

which, in view of (5.12) and the uniform boundedness of $\left(\nabla u_{\varepsilon}\right)_{\varepsilon}$ in $L^{p}\left(Q ; \mathbb{R}^{n}\right)$, results in

$$
\lim _{\varepsilon \rightarrow 0} \int_{\varepsilon Y_{\text {soft }} \cap Q_{\varepsilon}} \mathcal{M}\left(\nabla u_{\varepsilon}\right) \mathrm{d} x=\mathcal{M}\left(F_{\lambda}\right) .
$$

Finally, we combine (5.13) with (5.12) and (5.19) and exploit the continuity of $g$ as a convex function to arrive at (5.11). This concludes the proof of the lower bound. 
Remark 5.4. (a) Step 1 can be performed as above for any open and bounded set $\Omega$, meaning that the restriction to a flat, cross-section Lipschitz domain is not necessary for the construction of a sequence satisfying (5.7).

(b) Note that the recovery sequence constructed in Step 1 can be assumed to have the same boundary values as $v_{\varepsilon}^{F}$, i.e. $u_{\varepsilon}-v_{\varepsilon}^{F} \in W_{0}^{1, p}\left(\Omega ; \mathbb{R}^{n}\right)$. Indeed, the small-scale oscillations glued onto the laminate $v_{\varepsilon}^{F}$ for sufficiently small $\varepsilon$ can be adapted outside of $\{x \in \Omega: \operatorname{dist}(x, \partial \Omega)>2 \varepsilon\}$ to vanish on $\{x \in \Omega$ : $\operatorname{dist}(x, \partial \Omega)<\varepsilon\}$. This modification affects neither the convergence of $\left(u_{\varepsilon}\right)_{\varepsilon}$ nor of $\left(E_{\varepsilon}\left(u_{\varepsilon}\right)\right)_{\varepsilon}$.

Based on the findings of Part II for the affine case, we will now prove the homogenization $\Gamma$-convergence result for general limit functions.

Proof of Theorem 5.2 (Part III): General case. Let $u \in W^{1, p}\left(\Omega ; \mathbb{R}^{n}\right)$ be such that $u(x)=R(x) x+b(x)$ for $x \in \Omega$, where $R \in W^{1, p}(\Omega ; S O(n))$ and $b \in$ $W^{1, p}\left(\Omega ; \mathbb{R}^{n}\right)$ satisfy $\nabla^{\prime} R=0$ and $\nabla^{\prime} b=0$. As in the previous parts, we have arranged the arguments in several steps, numbered consecutively.

Step 3: Existence of a recovery sequence We aim to find a sequence $\left(u_{\varepsilon}\right)_{\varepsilon} \subset$ $W^{1, p}\left(\Omega ; \mathbb{R}^{n}\right)$ such that $u_{\varepsilon} \rightarrow u$ in $W^{1, p}\left(\Omega ; \mathbb{R}^{n}\right)$ and $\lim \sup _{\varepsilon \rightarrow 0} E_{\varepsilon}\left(u_{\varepsilon}\right) \leqq E_{\text {hom }}(u)$. The idea behind the construction of a recovery sequence for $u$ is to use the approximating sequence from Proposition 4.1 and to perturb it in the softer layers by suitably relaxing microstructures that guarantee the optimal energy. To obtain these perturbations, the results from Step 1 (Part II) are applied to piecewise affine approximations of $u$.

Step 3a: Piecewise constant approximation of $\nabla u$. Recall that the gradient of $u$ is

$$
\nabla u=R+\left(\partial_{n} R\right) x \otimes e_{n}+d \otimes e_{n} .
$$

First we approximate the functions in (5.20), that is $d, \partial_{n} R, R$, and the identity $\operatorname{map}_{\mathbb{R}^{n}}: x \mapsto x$, by simple functions. Indeed, by following standard constructions (e.g. [3, Theorem 1.2]), it is not hard to see that uniform approximation of the continuous function $R$ is possible while preserving the values in $S O(n)$. Without loss of generality, we may assume that all four approximations above have a common partition of $\Omega$. Due to the globally one-dimensional character of $d, \partial_{n} R$ and $R$, the elements of the partition that do not intersect with $\partial \Omega$ can be assumed to be cubes aligned with the coordinate axes. To be precise, for every $\delta>0$ there are finitely many cubes $Q_{\delta}^{i} \subset \mathbb{R}^{n}$, which we index by $I_{\delta}$, with maximal side length $\delta$ such that $\left|\Omega \backslash \bigcup_{i \in I_{\delta}} Q_{\delta}^{i}\right|=0$ and $Q_{\delta}^{i} \cap \Omega \neq \emptyset$ for $i \in I_{\delta}$, and $d_{\delta}^{i}, \xi_{\delta}^{i} \in \mathbb{R}^{n}$, $S_{\delta}^{i} \in \mathbb{R}^{n \times n}$, and $R_{\delta}^{i} \in S O(n)$ such that the simple functions defined by

$$
\begin{aligned}
& R_{\delta}=\sum_{i \in I_{\delta}} R_{\delta}^{i} \mathbb{1}_{Q_{\delta}^{i} \cap \Omega}, \quad d_{\delta}=\sum_{i \in I_{\delta}} d_{\delta}^{i} \mathbb{1}_{Q_{\delta}^{i} \cap \Omega}, \\
& S_{\delta}=\sum_{i \in I_{\delta}} S_{\delta}^{i} \mathbb{1}_{Q_{\delta}^{i} \cap \Omega} \text { and } \quad \xi_{\delta}=\sum_{i \in I_{\delta}} \xi_{\delta}^{i} \mathbb{1}_{Q_{\delta}^{i} \cap \Omega},
\end{aligned}
$$


satisfy

$$
\begin{aligned}
& \left\|R_{\delta}-R\right\|_{L^{\infty}\left(\Omega ; \mathbb{R}^{n \times n}\right)}+\left\|d_{\delta}-d\right\|_{L^{p}\left(\Omega ; \mathbb{R}^{n}\right)} \\
& \quad+\left\|S_{\delta}-\partial_{n} R\right\|_{L^{p}\left(\Omega ; \mathbb{R}^{n}\right)}+\left\|\xi_{\delta}-\mathrm{id}_{\mathbb{R}^{n}}\right\|_{L^{\infty}\left(\Omega ; \mathbb{R}^{n}\right)}<\delta .
\end{aligned}
$$

Consider the piecewise constant function $U_{\delta} \in L^{\infty}\left(\Omega ; \mathbb{R}^{n \times n}\right)$ defined by

$$
U_{\delta}=R_{\delta}+S_{\delta} \xi_{\delta} \otimes e_{n}+d_{\delta} \otimes e_{n}=\sum_{j \in I_{\delta}} U_{\delta}^{i} \mathbb{1}_{Q_{\delta}^{i} \cap \Omega}
$$

where $U_{\delta}^{i}=R_{\delta}^{i}+S_{\delta}^{i} \xi_{\delta}^{i} \otimes e_{n}+d_{\delta}^{i} \otimes e_{n} \in \mathcal{A}$ for $i \in I_{\delta}$. Then,

$$
\left\|U_{\delta}-\nabla u\right\|_{L^{p}\left(\Omega ; \mathbb{R}^{n \times n}\right)} \leqq C \delta,
$$

with a constant $C>0$ independent of $\delta$. Indeed, in view of (5.21) and (5.20), this is an immediate consequence of the estimate

$$
\begin{aligned}
\left\|U_{\delta}-\nabla u\right\|_{L^{p}\left(\Omega ; \mathbb{R}^{n \times n}\right)} \leqq & \left\|R_{\delta}-R\right\|_{L^{\infty}\left(\Omega ; \mathbb{R}^{n \times n}\right)}+\operatorname{diam}(\Omega)\left\|S_{\delta}-\partial_{n} R\right\|_{L^{p}\left(\Omega ; \mathbb{R}^{n \times n}\right)} \\
& +\left\|\partial_{n} R\right\|_{L^{p}\left(\Omega ; \mathbb{R}^{n \times n}\right)}\left\|\xi_{\delta}-\operatorname{id}_{\mathbb{R}^{n}}\right\|_{L^{p}\left(\Omega ; \mathbb{R}^{n}\right)} \\
& +\left\|d_{\delta}-d\right\|_{L^{p}\left(\Omega ; \mathbb{R}^{n}\right)} .
\end{aligned}
$$

Step 3b: Locally optimal microstructure By Step 1 (Part II), where recovery sequences in the affine case were established, we can find under consideration of Remark 5.4a) on each $Q_{\delta}^{i} \cap \Omega$ with $\delta>0$ and $i \in I_{\delta}$ a sequence $\left(u_{\delta, \varepsilon}^{i}\right)_{\varepsilon} \subset$ $W^{1, p}\left(Q_{\delta}^{i} \cap \Omega ; \mathbb{R}^{n}\right)$ such that $\nabla u_{\delta, \varepsilon}^{i} \rightarrow U_{\delta}$ in $L^{p}\left(Q_{\delta}^{i} \cap \Omega ; \mathbb{R}^{n \times n}\right)$ as $\varepsilon \rightarrow 0$ and

$$
\begin{aligned}
\lim _{\varepsilon \rightarrow 0} \int_{Q_{\delta}^{i} \cap \Omega} W_{\varepsilon}^{\alpha}\left(x, \nabla u_{\delta, \varepsilon}^{i}\right) \mathrm{d} x & =\lim _{\varepsilon \rightarrow 0} \int_{\varepsilon Y_{\text {soft }} \cap Q_{\delta}^{i} \cap \Omega} W_{\text {soft }}\left(\nabla u_{\delta, \varepsilon}^{i}\right) \mathrm{d} x \\
& =\int_{Q_{\delta}^{i} \cap \Omega} W_{\text {hom }}\left(U_{\delta}\right) \mathrm{d} x .
\end{aligned}
$$

Now, with $w_{\delta, \varepsilon}^{i}:=v_{\varepsilon}^{U_{\delta}^{i}} \in W^{1, \infty}\left(Q_{\delta}^{i} \cap \Omega ; \mathbb{R}^{n}\right)$ a laminate as introduced in (4.4), let

$$
\varphi_{\delta, \varepsilon}^{i}=u_{\delta, \varepsilon}^{i}-w_{\delta, \varepsilon}^{i} \quad \text { on } Q_{\delta}^{i} \cap \Omega
$$

According to Remark $5.4 \mathrm{~b}$ ), we may assume that the boundary values of $u_{\delta, \varepsilon}^{i}$ and $w_{\delta, \varepsilon}^{i}$ coincide, which entails that $\varphi_{\delta, \varepsilon}^{i} \in W_{0}^{1, p}\left(Q_{\delta}^{i} \cap \Omega ; \mathbb{R}^{n}\right)$. Let us join these local components together in one function $\varphi_{\delta, \varepsilon} \in W_{0}^{1, p}\left(\Omega ; \mathbb{R}^{n}\right)$ given by

$$
\varphi_{\delta, \varepsilon}=\sum_{i \in I_{\delta}} \varphi_{\delta, \varepsilon}^{i} \mathbb{1}_{Q_{\delta}^{i} \cap \Omega} .
$$

Note that by construction $\varphi_{\delta, \varepsilon}=0$ in $\varepsilon Y_{\text {stiff }} \cap \Omega$. Moreover,

$$
\nabla \varphi_{\delta, \varepsilon} \rightarrow 0 \quad \text { in } L^{p}\left(\Omega ; \mathbb{R}^{n \times n}\right) \text { as } \varepsilon \rightarrow 0,
$$


and $\left\|\nabla \varphi_{\delta, \varepsilon}\right\|_{L^{p}\left(\Omega ; \mathbb{R}^{n \times n)}\right.}$ is uniformly bounded with respect to $\varepsilon$ and $\delta$. In analogy to $(5.25)$ we define for later reference the map of local laminates

$$
w_{\varepsilon, \delta}=\sum_{i \in I_{\delta}} w_{\delta, \varepsilon}^{i} \mathbb{1}_{Q_{\delta}^{i} \cap \Omega} \in L^{\infty}\left(\Omega ; \mathbb{R}^{n}\right)
$$

Since the homogenized energy density $W_{\text {hom }}$ satisfies the local Lipschitz condition (5.6) according to Remark 5.3(c), we infer along with (5.23) and Hölder's inequality that

$$
\begin{aligned}
\int_{\Omega} W_{\text {hom }}\left(U_{\delta}\right) \mathrm{d} x & \leqq \int_{\Omega} W_{\text {hom }}(\nabla u) \mathrm{d} x+C\left\|U_{\delta}-\nabla u\right\|_{L^{p}\left(\Omega ; \mathbb{R}^{n \times n}\right)} \\
& \leqq \int_{\Omega} W_{\mathrm{hom}}(\nabla u) \mathrm{d} x+C \delta
\end{aligned}
$$

Summing over all $i \in I_{\delta}$ in (5.24) and taking the limit $\varepsilon \rightarrow 0$ gives that

$$
\begin{aligned}
\limsup _{\varepsilon \rightarrow 0} \int_{\Omega} W_{\varepsilon}^{\alpha}\left(x, U_{\delta, \varepsilon}\right) \mathrm{d} x & =\limsup _{\varepsilon \rightarrow 0} \int_{\varepsilon Y_{\mathrm{soft}} \cap \Omega} W_{\text {soft }}\left(U_{\delta, \varepsilon}\right) \mathrm{d} x \\
& \leqq \int_{\Omega} W_{\mathrm{hom}}(\nabla u) \mathrm{d} x+C \delta
\end{aligned}
$$

where $U_{\varepsilon, \delta}=\sum_{i \in I_{\delta}} \nabla u_{\delta, \varepsilon}^{i} \mathbb{1}_{Q_{\delta}^{i} \cap \Omega}$.

Step 3c: Optimal construction with admissible gradient structure After diagonalization, the functions $U_{\varepsilon, \delta(\varepsilon)}$ would define a recovery sequence as desired, provided they have gradient structure, i.e., there is a potential $u_{\varepsilon} \in W^{1, p}\left(\Omega ; \mathbb{R}^{n}\right)$ with $\nabla u_{\varepsilon}=U_{\varepsilon, \delta(\varepsilon)}$. Due to incompatibilities at the interfaces between neighboring cubes, however, this can in general not be expected. To overcome this issue and to obtain an admissible recovery sequence, we discard the local laminates $w_{\varepsilon, \delta}$ from (5.27), and instead add the locally optimal microstructures $\varphi_{\delta, \varepsilon}$ onto the functions $v_{\varepsilon}$, which result from Proposition 4.1 applied to $u$.

More precisely, applying Proposition 4.1 to the given $u$ provides us with an approximating sequence in $W^{1, p}\left(\Omega ; \mathbb{R}^{n}\right)$ with useful properties, which we call $\left(v_{\varepsilon}\right)_{\varepsilon}$. In particular,

$$
\nabla v_{\varepsilon} \rightarrow \nabla u \text { in } L^{p}\left(\Omega ; \mathbb{R}^{n}\right),
$$

$\nabla v_{\varepsilon} \in S O(n)$ a.e. in $\varepsilon Y_{\text {stiff }} \cap \Omega$,

$$
\left\|\nabla v_{\varepsilon}-(\nabla u)_{\lambda}\right\|_{L^{p}\left(\varepsilon Y_{\mathrm{soft}} \cap \Omega ; \mathbb{R}^{n \times n}\right)} \rightarrow 0
$$

with $(\nabla u)_{\lambda}$ as in (4.5).

Let $u_{\delta, \varepsilon} \in W^{1, p}\left(\Omega ; \mathbb{R}^{n}\right)$ be given by

$$
u_{\delta, \varepsilon}=v_{\varepsilon}+\varphi_{\delta, \varepsilon}-f_{\Omega} v_{\varepsilon}+\varphi_{\delta, \varepsilon}-u \mathrm{~d} x
$$


Next, we estimate the energetic error brought about by replacing $w_{\varepsilon, \delta}$ in Step $3 \mathrm{~b}$ with $v_{\varepsilon}$. By $(H 3)$, Hölder's inequality and the above definitions,

$$
\begin{aligned}
& \int_{\varepsilon Y_{\text {soft }} \cap \Omega}\left|W_{\text {soft }}\left(U_{\delta, \varepsilon}\right)-W_{\text {soft }}\left(\nabla u_{\delta, \varepsilon}\right)\right| \mathrm{d} x \\
& \leq L\left\|1+\left|U_{\delta, \varepsilon}\right|^{p-1}+\left|\nabla u_{\delta, \varepsilon}\right|^{p-1}\right\|_{L^{\frac{p}{p-1}}\left(\varepsilon Y_{\text {soft }} \cap \Omega\right)}\left\|U_{\delta, \varepsilon}-\nabla u_{\delta, \varepsilon}\right\|_{L^{p}\left(\varepsilon Y_{\text {soft }} \cap \Omega ; \mathbb{R}^{n \times n}\right)} \\
& \leq C\left(1+\left\|\nabla v_{\varepsilon}\right\|_{L^{p}\left(\Omega ; \mathbb{R}^{n}\right)}+\left\|\left(U_{\delta}\right)_{\lambda}\right\|_{L^{p}\left(\Omega ; \mathbb{R}^{n \times n}\right)}\right. \\
& \left.\quad+\left\|\nabla \varphi_{\varepsilon, \delta}\right\|_{L^{p}\left(\Omega ; \mathbb{R}^{n}\right)}\right)\left\|\left(U_{\delta}\right)_{\lambda}-\nabla v_{\varepsilon}\right\|_{L^{p}\left(\varepsilon Y_{\text {soft }} \cap \Omega ; \mathbb{R}^{n \times n}\right)}
\end{aligned}
$$

with $C>0$ independent of $\varepsilon$ and $\delta$. The first factor in the last line of (5.31) is uniformly bounded (with respect to $\delta$ and $\varepsilon$ ) as a consequence of (5.29), (5.23) and the remark below (5.26). The second factor can be controlled with the help of (5.30) and the following estimate, which exploits (5.21) and (5.23):

$$
\begin{aligned}
& \left\|(\nabla u)_{\lambda}-\left(U_{\delta}\right)_{\lambda}\right\|_{L^{p}\left(\varepsilon Y_{\mathrm{soft}} \cap \Omega ; \mathbb{R}^{n \times n}\right)} \\
& \quad \leqq\left(1-\frac{1}{\lambda}\right)\left\|R-R_{\delta}\right\|_{L^{\infty}\left(\Omega ; \mathbb{R}^{n \times n}\right)}+\frac{1}{\lambda}\left\|\nabla u-U_{\delta}\right\|_{L^{p}\left(\Omega ; \mathbb{R}^{n \times n}\right)} \leqq C \delta .
\end{aligned}
$$

Thus,

$$
\begin{aligned}
& \int_{\varepsilon Y_{\text {soft }} \cap \Omega}\left|W_{\text {soft }}\left(U_{\delta, \varepsilon}\right)-W_{\text {soft }}\left(\nabla u_{\delta, \varepsilon}\right)\right| \mathrm{d} x \\
& \quad \leqq C\left(\left\|(\nabla u)_{\lambda}-\nabla v_{\varepsilon}\right\|_{L^{p}\left(\varepsilon Y_{\text {soft }} \cap \Omega ; \mathbb{R}^{n \times n}\right)}+\delta\right) .
\end{aligned}
$$

Step 3d: Diagonalization As both $U_{\varepsilon, \delta}$ and $\nabla u_{\delta, \varepsilon}$ lie in $S O(n)$ almost everywhere on the stiff layers, (5.28) in combination with (5.33), (5.30) and (H2) yields that

$$
\limsup _{\varepsilon \rightarrow 0} E_{\varepsilon}\left(u_{\varepsilon, \delta}\right) \leqq \int_{\Omega} W_{\text {hom }}(\nabla u) \mathrm{d} x+C \delta .
$$

Moreover, we derive from (5.29) and (5.26) that $\nabla u_{\varepsilon, \delta} \rightarrow \nabla u$ in $L^{p}\left(\Omega ; \mathbb{R}^{n}\right)$ as $\varepsilon \rightarrow 0$ for every $\delta$. After exploiting Poincaré's inequality, the compact embedding of $W^{1, p}$ into $L^{p}$, and the Urysohn subsequence principle it follows then that $u_{\varepsilon, \delta} \rightarrow u$ in $L^{p}\left(\Omega ; \mathbb{R}^{n}\right)$ as $\varepsilon \rightarrow 0$.

Finally, the diagonalization lemma by Аттоuсн (see e.g. [4, Lemma 1.15, Corollary 1.16]) guarantees the existence of a sequence $\delta(\varepsilon)$ such that $u_{\varepsilon}:=$ $u_{\varepsilon, \delta(\varepsilon)} \in W^{1, p}\left(\Omega ; \mathbb{R}^{n}\right)$ satisfies that

$$
\limsup _{\varepsilon \rightarrow 0} E_{\varepsilon}\left(u_{\varepsilon}\right) \leqq \int_{\Omega} W_{\text {hom }}(\nabla u) \mathrm{d} x
$$

and that $u_{\varepsilon} \rightarrow u$ in $L^{p}\left(\Omega ; \mathbb{R}^{n}\right)$. This shows that $\left(u_{\varepsilon}\right)_{\varepsilon}$ is a recovery sequence for $u$ as stated.

Step 4: Lower bound. Let $\left(u_{\varepsilon}\right)_{\varepsilon} \subset W^{1, p}\left(\Omega ; \mathbb{R}^{n}\right)$ be a sequence of uniformly bounded energy, i.e., $E_{\varepsilon}\left(u_{\varepsilon}\right)<C$ for all $\varepsilon>0$, such that $u_{\varepsilon} \rightarrow u$ in $W^{1, p}\left(\Omega ; \mathbb{R}^{n}\right)$ 
for some $u \in W^{1, p}\left(\Omega ; \mathbb{R}^{n}\right)$. By Theorem 3.1, $\nabla u$ has the form (4.1). We will show that

$$
\liminf _{\varepsilon \rightarrow 0} \int_{\varepsilon Y_{\text {soft }} \cap \Omega} W_{\text {soft }}\left(\nabla u_{\varepsilon}\right) \mathrm{d} x \geqq \int_{\Omega} \lambda W_{\text {soft }}^{\mathrm{qc}}\left((\nabla u)_{\lambda}\right) \mathrm{d} x=\int_{\Omega} W_{\text {hom }}(\nabla u) \mathrm{d} x,
$$

which implies the desired liminf-inequality $\liminf _{\varepsilon \rightarrow 0} E_{\varepsilon}\left(u_{\varepsilon}\right) \geqq E_{\text {hom }}(u)$.

To tie this general case to the affine one in Step 2, we adjust to our specific situation a common approximation strategy (see e.g. [47, Theorem 1.3]) based on comparison sequences that involve elements of the constructed recovery sequences. Note that there is no need for the comparison sequence to have full gradient structure, which allows us to argue separately on each piece of the piecewise constant approximation of $\nabla u$.

Step 4a: Construction of a comparison sequence. First, we approximate $\nabla u$ by piecewise constant functions $U_{\delta}$ as in Step 3a, see (5.22) and (5.23). For $\varepsilon, \delta>0$ let $w_{\varepsilon, \delta}$ and $v_{\varepsilon}$ be as in Step 3c. Recall that for any $\delta>0$ and $i \in I_{\delta}$,

$$
\nabla w_{\delta, \varepsilon}^{i} \rightarrow U_{\delta}^{i} \quad \text { in } L^{p}\left(Q_{\delta}^{i} ; \mathbb{R}^{n \times n}\right) \text { as } \varepsilon \rightarrow 0,
$$

and that the sequence $\left(v_{\varepsilon}\right)_{\varepsilon} \subset W^{1, p}\left(\Omega ; \mathbb{R}^{n}\right)$ satisfies (5.29) and (5.30). Moreover,

$$
\left\|\nabla v_{\varepsilon}-R\right\|_{L^{p}\left(\varepsilon Y_{\text {stiff }} \cap \Omega ; \mathbb{R}^{n \times n}\right)} \rightarrow 0 \text { as } \varepsilon \rightarrow 0,
$$

in view of Proposition 4.1.

Now let us introduce

$$
z_{\delta, \varepsilon}=u_{\varepsilon}-v_{\varepsilon}+w_{\delta, \varepsilon}+f_{\Omega} v_{\varepsilon}-w_{\delta, \varepsilon} \mathrm{d} x .
$$

These functions have vanishing mean value on $\Omega$ and satisfy $z_{\delta, \varepsilon}^{i}=\left.z_{\delta, \varepsilon}\right|_{Q_{\delta}^{i}} \in$ $W^{1, p}\left(Q_{\delta}^{i} ; \mathbb{R}^{n}\right)$ for any $i \in I_{\delta}$. Due to (5.35), (5.29) and the assumption on the weak convergence of $\left(u_{\varepsilon}\right)_{\varepsilon}$, it follows for every $\delta>0$ that

$$
\nabla z_{\delta, \varepsilon}^{i}=\nabla u_{\varepsilon}-\nabla v_{\varepsilon}+\nabla w_{\delta, \varepsilon}^{i} \rightarrow U_{\delta}^{i} \quad \text { in } L^{p}\left(Q_{\delta}^{i} ; \mathbb{R}^{n}\right) \quad \text { as } \varepsilon \rightarrow 0 .
$$

Hence, as a consequence of the result in the affine case (see Step 2, Part II), applied to the restriction of $z_{\delta, \varepsilon}$ to any cuboid $Q_{\delta}^{i}$ with $i \in \tilde{I}_{\delta}:=\left\{i \in I_{\delta}: Q_{\delta}^{i} \subset \subset\right.$ $Q\}$, we deduce that

$$
\liminf _{\varepsilon \rightarrow 0} \int_{\varepsilon Y_{\text {soft }} \cap Q_{\delta}^{i}} W_{\varepsilon}^{\alpha}\left(x, \nabla z_{\delta, \varepsilon}^{i}\right) \mathrm{d} x \geqq \int_{Q_{\delta}^{i}} W_{\text {hom }}\left(U_{\delta}^{i}\right) \mathrm{d} x .
$$

In fact, if

$$
\left\|\operatorname{dist}\left(\nabla z_{\delta, \varepsilon}^{i}, S O(n)\right)\right\|_{L^{p}\left(\varepsilon Y_{\text {stiff }} \cap Q_{\delta}^{i}\right)} \rightarrow 0
$$

as $\varepsilon \rightarrow 0$, one can follow the reasoning of Step 2 in Part II to see that even

$$
\liminf _{\varepsilon \rightarrow 0} \int_{\varepsilon Y_{\text {soft }} \cap Q_{\delta}^{i}} W_{\text {soft }}\left(\nabla z_{\delta, \varepsilon}^{i}\right) \mathrm{d} x \geqq \int_{Q_{\delta}^{i}} W_{\text {hom }}\left(U_{\delta}^{i}\right) \mathrm{d} x .
$$


To verify (5.37) for $i \in \tilde{I}_{\delta}$, we mimic the arguments leading to (5.15) on the cuboid $Q_{\delta}^{i} \subset \subset \Omega$. This implies in particular that

$$
\left\|\nabla u_{\varepsilon}-R\right\|_{L^{p}\left(\varepsilon Y_{\text {stiff }} \cap Q_{\delta}^{i} ; \mathbb{R}^{n \times n}\right)} \rightarrow 0 .
$$

Then,

$$
\begin{aligned}
& \left\|\nabla z_{\delta, \varepsilon}^{i}-R_{\delta}^{i}\right\|_{L^{p}\left(\varepsilon Y_{\text {stiff }} \cap Q_{\delta}^{i} ; \mathbb{R}^{n \times n}\right)} \\
& \quad=\left\|\nabla z_{\delta, \varepsilon}^{i}-\nabla w_{\delta, \varepsilon}^{i}\right\|_{L^{p}\left(\varepsilon Y_{\text {stiff }} \cap Q_{\delta}^{i} ; \mathbb{R}^{n \times n}\right)} \\
& \quad=\left\|\nabla u_{\varepsilon}-\nabla v_{\varepsilon}\right\|_{L^{p}\left(\varepsilon Y_{\text {stiff }} \cap Q_{\delta}^{i} ; \mathbb{R}^{n \times n}\right)} \\
& \quad \leqq\left\|\nabla u_{\varepsilon}-R\right\|_{L^{p}\left(\varepsilon Y_{\text {stiff }} \cap Q_{\delta}^{i} ; \mathbb{R}^{n \times n}\right)}+\left\|R-\nabla v_{\varepsilon}\right\|_{L^{p}\left(\varepsilon Y_{\text {stiff }} \cap Q_{\delta}^{i} ; \mathbb{R}^{n \times n}\right)},
\end{aligned}
$$

which, in light of (5.39) and (5.36), gives (5.37).

Step 4b: Energy estimates For the homogenized energy, we derive from the local Lipschitz continuity of $W_{\text {hom }}$ (cf. Remark 5.3(c)), along with (5.23) and Hölder's inequality, that

$$
\int_{\Omega}\left|W_{\text {hom }}\left(U_{\delta}\right)-W_{\text {hom }}(\nabla u)\right| \mathrm{d} x \leqq C\left\|U_{\delta}-\nabla u\right\|_{L^{p}\left(\Omega ; \mathbb{R}^{n \times n}\right)}<C \delta .
$$

Furthermore, with $(H 2)$ and the uniform $L^{p}$-bounds on $\nabla u_{\varepsilon}$ and $\nabla w_{\delta, \varepsilon}^{i}$, we have for any $i \in I_{\delta}$,

$$
\begin{aligned}
& \int_{\varepsilon Y_{\text {soft }} \cap Q_{\delta}^{i}}\left|W_{\text {soft }}\left(\nabla z_{\delta, \varepsilon}^{i}\right)-W_{\text {soft }}\left(\nabla u_{\varepsilon}\right)\right| \\
& \quad=\int_{\varepsilon Y_{\text {soft }} \cap Q_{\delta}^{i}}\left|W_{\text {soft }}\left(\nabla u_{\varepsilon}-\nabla v_{\varepsilon}+\nabla w_{\delta, \varepsilon}\right)-W_{\text {soft }}\left(\nabla u_{\varepsilon}\right)\right| \mathrm{d} x \\
& \quad \leqq C\left\|\nabla v_{\varepsilon}-\nabla w_{\delta, \varepsilon}\right\|_{L^{p}\left(\varepsilon Y_{\text {soft }} \cap Q_{\delta}^{i} ; \mathbb{R}^{n \times n}\right)} \\
& \quad \leqq C\left(\left\|\nabla v_{\varepsilon}-(\nabla u)_{\lambda}\right\|_{L^{p}\left(\varepsilon Y_{\text {soft }} \cap Q_{\delta}^{i} ; \mathbb{R}^{n \times n}\right)}+\left\|(\nabla u)_{\lambda}-\left(U_{\delta}\right)_{\lambda}\right\|_{L^{p}\left(\varepsilon Y_{\text {soft }} \cap Q_{\delta}^{i} ; \mathbb{R}^{n \times n}\right)}\right) .
\end{aligned}
$$

Due to (5.30), the first expression on the right hand side converges to zero as $\varepsilon \rightarrow$ 0 , while the second can be estimated from above by $\delta$ by (5.32). Considering (5.38), we conclude after summing over $i \in \tilde{I}_{\delta}$ that

$$
\liminf _{\varepsilon \rightarrow 0} \int_{\varepsilon Y_{\text {soft }} \cap \Omega_{\delta}} W_{\text {soft }}\left(\nabla u_{\varepsilon}\right) \mathrm{d} x \geqq \int_{\Omega_{\delta}} W_{\text {hom }}(\nabla u) \mathrm{d} x-C \delta,
$$

where $\Omega_{\delta}=\bigcup_{i \in \tilde{I}_{\delta}} Q_{\delta}^{i}$. Since $\left|\Omega \backslash \Omega_{\delta}\right| \rightarrow 0$ by construction, passing to the limit $\delta \rightarrow 0$ establishes (5.34), which concludes the proof.

As the next remark shows, the homogenized energy density $W_{\text {hom }}$ from (5.4) coincides with the single-cell formula arising from a related model without elasticity (" $\alpha=\infty$ ") on the stiff layers. This observation indicates that microstructures developing over multiple cells, as they are to be expected in general homogenization problems with non-convex energy densities (cf. [47] and more recently [5]), do not occur. They are indeed inhibited by the presence of the stiff horizontal layers. 
Remark 5.5. With $W_{\text {soft }}$ satisfying $(H 1)-(H 3)$ and $W_{\text {rig }}(F)=\chi_{S O(n)}(F)$ for $F \in$ $\mathbb{R}^{n \times n}$, let $\bar{W}: \Omega \times \mathbb{R}^{n \times n} \rightarrow[0, \infty]$ be given by

$$
\bar{W}(x, F)= \begin{cases}W_{\text {rig }}(F) & \text { for } x \in Y_{\text {stiff }} \cap \Omega, \\ W_{\text {soft }}(F) & \text { for } x \in Y_{\text {soft }} \cap \Omega,\end{cases}
$$

and denote by $\bar{W}_{\text {cell }}$ the cell formula associated with $\bar{W}$, i.e.,

$$
\bar{W}_{\text {cell }}(F)=\inf _{\psi \in W_{\#}^{1, p}\left(Y ; \mathbb{R}^{n}\right)} f_{Y} \bar{W}(y, F+\nabla \psi) \mathrm{d} y, \quad F \in \mathbb{R}^{n \times n} .
$$

We will show that for $F \in \mathbb{R}^{n \times n}$,

$$
\bar{W}_{\text {cell }}(F)= \begin{cases}W_{\text {hom }}(F) & \text { for } F \in \mathcal{A}, \\ \infty & \text { otherwise }\end{cases}
$$

Indeed, if $\bar{W}_{\text {cell }}(F)<\infty$, there exists $\psi \in W_{\#}^{1, p}\left(Y ; \mathbb{R}^{n}\right)$ such that the expression $f_{Y} W_{\text {rig }}(y, F+\nabla \psi)$ d $y$ is finite. This implies $F+\nabla \psi \in S O(n)$ a.e. in $Y_{\text {stiff }}$, and we infer from Reshetnyak's theorem [53] (cf. also Theorem 3.3) that for some $R \in S O(n)$,

$$
F+\nabla \psi=R \quad \text { on } Y_{\text {stiff }} .
$$

Therefore, since $\psi$ is periodic, one obtains for $i=1, \ldots, n-1$ that

$$
F e_{i}=F e_{i}+\int_{Y} \partial_{i} \psi \mathrm{d} y=\int_{Y} R e_{i} \mathrm{~d} y=R e_{i},
$$

and hence, $F \in \mathcal{A}$ and in particular, $F=R+d \otimes e_{n}$ with $d \in \mathbb{R}^{n}$. By (5.41), $\nabla \psi=-d \otimes e_{n}$ on $Y_{\text {stiff }}$.

Considering the piecewise affine function $v \in W_{\#}^{1, \infty}\left(Y ; \mathbb{R}^{n}\right)$ with zero mean value and gradient

$$
\nabla v=\left(-\mathbb{1}_{Y_{\text {stiff }}}+\frac{1-\lambda}{\lambda} \mathbb{1}_{Y_{\text {soft }}}\right) d \otimes e_{n},
$$

we can find $\varphi \in W_{\#}^{1, p}\left(Y ; \mathbb{R}^{n}\right)$ such that $\nabla \varphi=0$ in $Y_{\text {stiff }}$ and $\psi$ is represented as $\psi=v+\varphi$. Thus,

$$
\begin{aligned}
& \inf _{\psi \in W_{\#}^{1, p}\left(Y ; \mathbb{R}^{n}\right)} f_{Y} \bar{W}(y, F+\nabla \psi) \mathrm{d} y \\
& =\inf \left\{\int_{Y_{\text {soft }}} W_{\text {soft }}(F+\nabla \psi) \mathrm{d} y: \psi \in W_{\#}^{1, p}\left(Y ; \mathbb{R}^{n}\right), \nabla \psi=-d \otimes e_{n} \text { on } Y_{\text {stiff }}\right\} \\
& =\inf \left\{\int_{Y_{\text {soft }}} W_{\text {soft }}\left(F+\frac{1-\lambda}{\lambda} d \otimes e_{n}+\nabla \varphi\right) \mathrm{d} y: \varphi \in W_{\#}^{1, p}\left(Y ; \mathbb{R}^{n}\right), \nabla \varphi=0 \text { on } Y_{\text {stiff }}\right\} \\
& =\inf \left\{\int_{Y_{\text {soft }}} \lambda W_{\text {soft }}\left(F_{\lambda}+\nabla \varphi\right) \mathrm{d} y: \varphi \in W_{\#}^{1, p}\left(Y ; \mathbb{R}^{n}\right), \varphi=0 \text { on } Y_{\text {stiff }}\right\} \\
& =\lambda \inf _{\phi \in W_{0}^{1, p}\left(Y_{\text {soft }} ; \mathbb{R}^{n}\right)} f_{Y_{\text {soft }}} W_{\text {soft }}\left(F_{\lambda}+\nabla \phi\right) \mathrm{d} y,
\end{aligned}
$$

where the last equality makes use of Remark $5.3 \mathrm{~b}$ ). This verifies (5.40). 
Acknowledgements. The authors would like to thank Georg Dolzmann for his valuable comments on FC's Ph.D. thesis, which helped to improve also the presentation of this manuscript. FC gratefully acknowledges a traveling grant by the DFG Graduiertenkolleg 1692 "Curvature, Cyles, and Cohomology". CK was partially supported by a Westerdijk Fellowship from Utrecht University.

Open Access This article is distributed under the terms of the Creative Commons Attribution 4.0 International License (http://creativecommons.org/licenses/by/4.0/), which permits unrestricted use, distribution, and reproduction in any medium, provided you give appropriate credit to the original author(s) and the source, provide a link to the Creative Commons license, and indicate if changes were made.

Publisher's Note Springer Nature remains neutral with regard to jurisdictional claims in published maps and institutional affiliations.

\section{Appendix A. Collected Auxiliary Results}

In the next lemma, we provide a type of reverse Poincaré inequality for special affine maps given as the difference of two rotations on a domain that is thin in one dimension. The special feature of this result (e.g. in comparison with classical Caccioppoli estimates for harmonic maps [39]) is that the constant can be chosen independently of the thickness of the domain in the $e_{n}$-direction.

Lemma A.1. For an integer $n \geqq 2$ let $P=O \times I$ with $O \subset \mathbb{R}^{n-1}$ an open cube of side length $l>0$ and $I \subset \mathbb{R}$ an interval of length $h>0$, and let $1 \leqq p<\infty$. Then there exists a constant $C>0$ depending only on $n$ and $p$ such that for all rotations $R_{1}, R_{2} \in S O(n)$ and translation vectors $d \in \mathbb{R}^{n}$,

$$
\int_{P}\left|\left(R_{2}-R_{1}\right) x+d\right|^{p} \mathrm{~d} x \geqq C l^{p}|P|\left|R_{2}-R_{1}\right|^{p} .
$$

Proof. We will prove the result for $p=1$, for general $p$ the statement then follows immediately from Hölder's inequality.

Moreover, without loss of generality let $R_{2}$ be the identity matrix $\mathbb{I}=\mathbb{I}_{n} \in$ $\mathbb{R}^{n \times n}$. We set $R=R_{1} \in S O(n)$ and write $A:=\mathbb{I}-R \in \mathbb{R}^{n \times n}$. Let $\bar{P}$ denote the translation of the open cuboid $P$ centered in the origin. The arguments below make use of the nested sets $\widehat{P} \subset Z \subset \bar{P}$, where $Z$ is the cylinder with circular cross section inscribed in $\bar{P}$ and $\widehat{P}$ is the largest centered, open cuboid contained in $Z$. Precisely,

$$
Z=B_{l / 2}^{n-1} \times\left(-\frac{h}{2}, \frac{h}{2}\right) \text { and } \widehat{P}=\left(-\frac{l}{2 \sqrt{n}}, \frac{l}{2 \sqrt{n}}\right)^{n-1} \times\left(-\frac{h}{2}, \frac{h}{2}\right),
$$

where $B_{r}^{n-1}$ the $(n-1)$-dimensional ball around the origin with radius $r$.

With this notation in place, we observe that

$$
\int_{P}|A x+d| \mathrm{d} x \geqq \int_{\bar{P}}|A x| \mathrm{d} x \geqq \int_{Z}|A x| \mathrm{d} x .
$$

To derive the desired estimate, we determine the singular values of $A$. It follows from the specific structure of $A$ that

$$
A^{T} A=2 \mathbb{I}-\left(R+R^{T}\right) .
$$


Considering that every $R \in S O(n)$ can be transformed into block diagonal form with the help of another rotation $U \in S O(n)$, i.e. there is an integer $k \leqq \frac{n}{2}$ and two-dimensional rotations $\Theta_{1}, \ldots, \Theta_{k} \in S O(2)$ such that

$$
R=U^{T} \operatorname{diag}\left(\Theta_{1}, \ldots, \Theta_{k}, \mathbb{I}_{n-2 k}\right) U,
$$

see e.g. [41, Satz 8.3.10], we conclude from the fact that the symmetric part of a two-dimensional rotation matrix is diagonal that $A^{T} A=U^{T} D U$, where

$$
D=2 \operatorname{diag}\left(1-\theta_{1}, 1-\theta_{1}, \ldots, 1-\theta_{k}, 1-\theta_{k}, 0, \ldots, 0\right) \in \mathbb{R}^{n \times n},
$$

with $\theta_{i}=\left(\Theta_{i}\right)_{11} \in[-1,1)$. One may assume without loss of generality that $\theta_{1} \leqq \theta_{2} \leqq \cdots \leqq \theta_{k}$, which implies that $2\left(1-\theta_{1}\right)$ is the largest eigenvalue of $A^{T} A$, and hence corresponds to the squared spectral norm of $A$. Since all norms on $\mathbb{R}^{n \times n}$ are equivalent, there is a constant $C=C(n)>0$ such that $\sqrt{2\left(1-\theta_{1}\right)} \geqq C|A|$, where $|\cdot|$ denotes the Frobenius norm. Hence,

$$
\begin{aligned}
\int_{Z}|A x| \mathrm{d} x & =\int_{Z} \sqrt{A^{T} A x \cdot x} \mathrm{~d} x=\int_{Z} \sqrt{D(U x) \cdot U x} \mathrm{~d} x \\
& \geqq \sqrt{2\left(1-\theta_{1}\right)} \int_{U Z} \sqrt{x_{1}^{2}+x_{2}^{2}} \mathrm{~d} x \geqq C|A| \int_{U Z} \sqrt{x_{1}^{2}+x_{2}^{2}} \mathrm{~d} x .
\end{aligned}
$$

In view of (A.1) and (A.2) it remains to show that

$$
\int_{U Z} \sqrt{x_{1}^{2}+x_{2}^{2}} \mathrm{~d} x \geqq C l|P|,
$$

with $C>0$ depending only on $n$. If $U=\mathbb{I}$, we simply neglect one of the two additive terms in the integrand, say $x_{2}^{2}$, and estimate that

$$
\int_{Z}\left|x_{1}\right| \mathrm{d} x \geqq \int_{\widehat{P}}\left|x_{1}\right| \mathrm{d} x=2 h\left(\frac{l}{\sqrt{n}}\right)^{n-2} \int_{0}^{\frac{l}{2 \sqrt{n}}} x_{1} \mathrm{~d} x_{1}=h\left(\frac{l}{\sqrt{n}}\right)^{n}=n^{-\frac{n}{2}} l|P| .
$$

For general $U$, our argument requires to select a suitable rotation of the plane spanned by the unit vectors $e_{1}$ and $e_{2}$ to guarantee that the axes of the rotated cylinder $U Z$ is orthogonal to $e_{1}$. More precisely, one observes that any planar rotation $S=\operatorname{diag}\left(\Sigma, \mathbb{I}_{n-2}\right)$ with $\Sigma \in S O(2)$ leaves the integral in (A.3) unchanged, and therefore

$$
\int_{U Z} \sqrt{x_{1}^{2}+x_{2}^{2}} \mathrm{~d} x=\int_{S U Z} \sqrt{x_{1}^{2}+x_{2}^{2}} \mathrm{~d} x \geqq \int_{S U Z}\left|x_{1}\right| \mathrm{d} x .
$$

Since the intersection of $\operatorname{span}\left\{e_{1}, e_{2}\right\}$ with the $(n-1)$-dimensional orthogonal complement of $\operatorname{span}\left\{U e_{n}\right\}$ is at least a one-dimensional subspace, we can choose a planar rotation $S$ such that $U e_{n} \cdot S^{T} e_{1}=0$, and thus $(S U)^{T} e_{1} \cdot e_{n}=0$. Then there exists $Q=\operatorname{diag}\left(\Xi, \mathbb{I}_{1}\right) \in S O(n)$ with $\Xi \in \mathrm{SO}(n-1)$ such that $Q^{T} e_{1}=(S U)^{T} e_{1}$, and

$$
\int_{S U Z}\left|x_{1}\right| \mathrm{d} x=\int_{Z}\left|S U x \cdot e_{1}\right| \mathrm{d} x=\int_{Z}\left|Q x \cdot e_{1}\right| \mathrm{d} x=\int_{Q Z}\left|x_{1}\right| \mathrm{d} x=\int_{Z}\left|x_{1}\right| \mathrm{d} x,
$$


where we have used the invariance of the cylinder $Z$ under rotations that leave the $x_{n}$-component unaffected. In view of (A.5) and (A.4) this shows (A.3), and hence, finishes the proof.

Next we give details on the extension result for locally one-dimensional functions in the $e_{n}$-direction used in Sections 3 and 4. Recall that for a bounded domain $\Omega \subset \mathbb{R}^{n}$, the smallest open cuboid containing $\Omega$ is denoted by $Q_{\Omega}$ and $Q_{\Omega}=O_{\Omega} \times J_{\Omega}$ with $O_{\Omega} \subset \mathbb{R}^{n-1}$ an open cuboid and an open interval $J_{\Omega} \subset \mathbb{R}$.

Lemma A.2. Let $\Omega \subset \mathbb{R}^{n}$ be a bounded, flat and cross-section connected Lipschitz domain. If $v \in W^{1, p}\left(\Omega ; \mathbb{R}^{m}\right)$ satisfies $\nabla^{\prime} v=0$, then $v$ can be extended to $Q_{\Omega}$ by a globally one-dimensional function in the $e_{n}$-direction $\tilde{v} \in W^{1, p}\left(Q_{\Omega} ; \mathbb{R}^{m}\right) \cap$ $C^{0}\left(Q_{\Omega} ; \mathbb{R}^{m}\right)$.

In particular, one can identify $v$ with the one-dimensional function $v \in W^{1, p}$ $\left(J_{\Omega} ; \mathbb{R}^{m}\right)$ defined by the identity $\tilde{v}(x)=v\left(x_{n}\right)$ for $x \in Q_{\Omega}$.

Proof. As pointed out at the beginning of Section 3, $v$ is locally one-dimensional in the $e_{n}$-direction, and hence, locally constant on any non-empty cross section $\Omega_{t}=H_{t} \cap \Omega=\left\{x \in \mathbb{R}^{n}: x_{n}=t\right\} \cap \Omega$. Since the latter are connected by assumption, it follows that $v$ is also globally one-dimensional in the $e_{n}$-direction.

We can now define an extension $\tilde{v}$ of $v$ to $Q_{\Omega}$ by setting

$$
\tilde{v}(x)=v(y) \text { with } y \in \Omega_{x_{n}}
$$

for $x \in Q_{\Omega}$. Observe that with $Q_{\Omega}$ the smallest open cuboid such that $\Omega \subset Q_{\Omega}$, the intersection $H_{x_{n}} \cap \Omega=\Omega_{x_{n}}$ is non-empty for all $x \in \Omega$. Clearly, $\tilde{v}$ is globally one-dimensional in the $e_{n}$-direction by definition. It therefore remains to prove that $\tilde{v} \in W^{1, p}\left(Q_{\Omega} ; \mathbb{R}^{m}\right)$ (for continuity one can then argue as in the first paragraph of Section 3).

To see this we will construct a sequence $w_{j} \in C^{\infty}\left(\overline{Q_{\Omega}} ; \mathbb{R}^{m}\right)$ that approximates $\tilde{v}$ in $W^{1, p}\left(Q_{\Omega} ; \mathbb{R}^{m}\right)$. Let $J_{\Omega}=(a, b)$ with $a, b \in \mathbb{R}, a<b$. Since $\Omega$ is a flat Lipschitz domain there exist $x_{a} \in \Omega_{a}$ and $x_{b} \in \Omega_{b}$ and balls $B_{r}\left(x_{a}\right)$ and $B_{r}\left(x_{b}\right)$ with radius $r>0$ such that $B_{r}\left(x_{a}\right) \cap Q_{\Omega} \subset \Omega$ and $B_{r}\left(x_{b}\right) \cap Q_{\Omega} \subset \Omega$. Exploiting further that $\Omega$ is open and connected, hence also path-connected, we can connect the edge points $x_{a}$ with $x_{b}$ by a $C^{1}$-curve $\gamma$ (after smoothing of a continuous curve). Moreover, one can be chosen $\gamma$ to be monotone in $x_{n}$ due to the cross-section connectedness of $\Omega$ and even strictly monotone, which implies that $\gamma$ is a regular curve, considering that $\Omega$ is open. After reparametrization we obtain

$$
\gamma \in C^{1}\left([a, b] ; \mathbb{R}^{n}\right) \text { with } \gamma(t) \in \Omega_{t} \text { for all } t \in[a, b] .
$$

For the composition $w=v \circ \gamma \in W^{1, p}\left(J_{\Omega} ; \mathbb{R}^{m}\right)$ there exist approximating functions $w_{j} \in C^{\infty}\left(\overline{J_{\Omega}} ; \mathbb{R}^{m}\right)$ such that $w_{j} \rightarrow w$ in $W^{1, p}(a, b)$. Without changing notation, let us identify $w_{j}$ and $w$ with their constant expansion in $x^{\prime}$, that is with elements in $W^{1, p}\left(Q_{\Omega} ; \mathbb{R}^{m}\right)$ and $C^{\infty}\left(\overline{Q_{\Omega}} ; \mathbb{R}^{m}\right)$, respectively. Finally, in view of (A.6) and (A.7),

$$
w_{j} \rightarrow w=v \circ \gamma=\tilde{v} \text { in } W^{1, p}\left(Q_{\Omega} ; \mathbb{R}^{m}\right),
$$

which shows that $\tilde{v} \in W^{1, p}\left(Q_{\Omega} ; \mathbb{R}^{m}\right)$ and concludes the proof. 
Remark A.3. (a) Since only local arguments have been used in the proof above, Lemma A.2 still holds if $W^{1, p}\left(\Omega ; \mathbb{R}^{m}\right)$ is replaced with $W_{\mathrm{loc}}^{1, p}\left(\Omega ; \mathbb{R}^{m}\right)$. In this case, it is even enough to require that $\Omega \subset \mathbb{R}^{n}$ is a bounded, cross-section connected domain.

(b) As Lemma A.2 relies on constant extensions only, changing the codomain of $v$ from $\mathbb{R}^{m}$ to $S O(n)$ does not change the statement.

\section{References}

1. Acerbi, E., Chindò Piat, V., Dal Maso, G., Percivale, D.: An extension theorem from connected sets, and homogenization in general periodic domains. Nonlinear Anal. 18(5), 481-496, 1992

2. Alt, H.W.: Linear Functional Analysis. Universitext. Springer, London. An applicationoriented introduction, Translated from the German edition by Robert Nürnberg 2016

3. Amann, H., Escher, J.: Analysis. II. Birkhäuser Verlag, Basel. Translated from the 1999 German original by Silvio Levy and Matthew Cargo 2008

4. Аттоuсн, H.: Variational Convergence for Functions and Operators. Applicable Mathematics Series. Pitman (Advanced Publishing Program), Boston 1984

5. BARChiesi, M., Gloria, A.: New counterexamples to the cell formula in nonconvex homogenization. Arch. Ration. Mech. Anal. 195(3), 991-1024, 2010

6. Barchiesi, M., LazZaroni, G., ZepPieri, C.I.: A bridging mechanism in the homogenization of brittle composites with soft inclusions. SIAM J. Math. Anal. 48(2), 11781209,2016

7. Braides, A.: Homogenization of some almost periodic coercive functional. Rend. Accad. Naz. Sci. XL Mem. Mat. (5) 9(1), 313-321, 1985

8. Braides, A.: Г-Convergence for Beginners. Number 22 in Oxford Lecture Series in Mathematics and Its Applications, 1st edn. Oxford University Press, Oxford 2005

9. Braides, A., FonseCa, I., LeONI, G.: $\mathcal{A}$-quasiconvexity: relaxation and homogenization. ESAIM Control Optim. Calc. Var. 5, 539-577, 2000

10. Braides, A., Garroni, A.: Homogenization of periodic nonlinear media with stiff and soft inclusions. Math. Models Methods Appl. Sci. 5(4), 543-564, 1995

11. Cagnetti, F., Scardia, L.: An extension theorem in SBV and an application to the homogenization of the Mumford-Shah functional in perforated domains. J. Math. Pures Appl. (9) 95(4), 349-381, 2011

12. Chambolle, A., Giacomini, A., Ponsiglione, M.: Piecewise rigidity. J. Funct. Anal. 244(1), 134-153, 2007

13. Cherdantsev, M., Cherednichenko, K., Neukamm, S.: High contrast homogenisation in nonlinear elasticity under small loads. Asymptot. Anal. 104(1-2), 67-102, 2017

14. Cherdantsev, M., Cherednichenko, K.D.: Two-scale $\Gamma$-convergence of integral functionals and its application to homogenisation of nonlinear high-contrast periodic composites. Arch. Ration. Mech. Anal. 204(2), 445-478, 2012

15. Christowiak, F.: Homogenization of Layered Materials with Stiff Components. Ph.D. thesis, Universität Regensburg, 2018

16. Christowiak, F., Kreisbeck, C.: Homogenization of layered materials with rigid components in single-slip finite crystal plasticity. Calc. Var. Partial Differ. Equ. 56(3), 75, 2017

17. Cioranescu, D., Damlamian, A., De Arcangelis, R.: Homogenization of integrals with pointwise gradient constraints via the periodic unfolding method. Ric. Mat. 55(1), 31-53, 2006

18. Cioranescu, D., Donato, P.: An Introduction to Homogenization, Volume 17 of Oxford Lecture Series in Mathematics and Its Applications. The Clarendon Press, Oxford University Press, New York 1999 
19. Conti, S.: Low-Energy Deformations of Thin Elastic Plates: Isometric Embeddings and Branching Patterns. Habilitation thesis, University of Leipzig, 2003

20. Conti, S., Dolzmann, G.: On the theory of relaxation in nonlinear elasticity with constraints on the determinant. Arch. Ration. Mech. Anal. 217(2), 413-437, 2015

21. Conti, S., Dolzmann, G., Kreisbeck, C.: Relaxation of a model in finite plasticity with two slip systems. Math. Models Methods Appl. Sci. 23(11), 2111-2128, 2013

22. Conti, S., Dolzmann, G., Müller, S.: Korn's second inequality and geometric rigidity with mixed growth conditions. Calc. Var. Partial Differ. Equ. 50(1-2), 437-454, 2014

23. Conti, S., Schweizer, B.: Rigidity and Gamma-convergence for solid-solid phase transitions with SO(2) invariance. Commun. Pure Appl. Math. 59(6), 830-868, 2006

24. Conti, S., Theil, F.: Single-slip elastoplastic microstructures. Arch. Ration. Mech. Anal. 178(1), 125-148, 2005

25. Dacorogna, B.: Direct Methods in the Calculus of Variations, Volume 78 of Applied Mathematical Sciences, 2nd edn. Springer, New York 2008

26. Dal Maso, G.: An Introduction to $\Gamma$-Convergence. Number 8 in Progress in Nonlinear Differential Equations and Their Applications. Birkhäuser, Boston 1993

27. Davoli, E., Ferreira, R., Kreisbeck, C.: Homogenization in BV of a model for layered composites in finite crystal plasticity. Preprint arXiv:1901.11517, 2019

28. De Giorgi, E.: Sulla convergenza di alcune successioni d'integrali del tipo dell' area. Rendiconti di Matematica e delle sue applicazioni 8(6), 277-294, 1975. Collection of articles dedicated to Mauro Picone on the occasion of his ninetieth birthday

29. De Giorgi, E., Franzoni, T.: Su un tipo di convergenza variazionale. Atti Accad. Naz. Lincei Rend. Cl. Sci. Fis. Mat. Natur. (8) 58(6), 842-850, 1975

30. Duerinckx, M., Gloria, A.: Stochastic homogenization of nonconvex unbounded integral functionals with convex growth. Arch. Ration. Mech. Anal. 221(3), 1511-1584, 2016

31. Faraco, D., Zhong, X.: Geometric rigidity of conformal matrices. Ann. Sc. Norm. Super. Pisa Cl. Sci. (5) 4(4), 557-585, 2005

32. Friedrich, M., SCHMIDT, B.: A quantitative geometric rigidity result in SBD. Preprint arXiv: $1503.06821,2015$

33. Friesecke, G., James, R.D., Müller, S.: A theorem on geometric rigidity and the derivation of nonlinear plate theory from three-dimensional elasticity. Commun. Pure Appl. Math. 55(11), 1461-1506, 2002

34. Friesecke, G., James, R.D., Müller, S.: A hierarchy of plate models derived from nonlinear elasticity by $\Gamma$-convergence. Arch. Ration. Mech. Anal. 180(2), 183-236, 2006

35. Gilbarg, D., Trudinger, N.S.: Elliptic Partial Differential Equations of Second Order. Classics in Mathematics, 2nd edn. Springer, Berlin 2001

36. Gurtin, M.E.: An Introduction to Continuum Mechanics. Number 158 in Mathematics in Science and Engineering. Academic Press, New York 1981

37. Hanche-Olsen, H., Holden, H.: The Kolmogorov-Riesz compactness theorem. Expo. Math. 28(4), 385-394, 2010

38. Iwaniec, T., Martin, G.: The Liouville theorem. In: Cazacu, C.A., Lehto, E.O. (eds.) Analysis and Topology, pp. 339-361. World Scientific Publishing, River Edge 1998

39. Iwaniec, T., Sbordone, C.: Caccioppoli estimates and very weak solutions of elliptic equations. Atti Accad. Naz. Lincei Cl. Sci. Fis. Mat. Natur. Rend. Lincei (9) Mat. Appl. 14(3), 189-205, 2004, 2003. Renato Caccioppoli and modern analysis

40. Koumatos, K., Rindler, F., Wiedemann, E.: Differential inclusions and Young measures involving prescribed Jacobians. SIAM J. Math. Anal. 47(2), 1169-1195, 2015

41. Kowalsky, H.-J., Michler, G.: Lineare Algebra. De Gruyter Lehrbuch. De Gruyter, Berlin, 2003. 12te Auflage.

42. KREISBECK, C., KRÖMER, S.: Heterogeneous thin films: combining homogenization and dimension reduction with directors. SIAM J. Math. Anal. 48(2), 785-820, 2016

43. Le Dret, H., Raoult, A.: The quasiconvex envelope of the Saint Venant-Kirchhoff stored energy function. Proc. R. Soc. Edinb. Sect. A 125(6), 1179-1192, 1995 
44. Leoni, G.: A First Course in Sobolev Spaces, Volume105 of Graduate Studies in Mathematics. American Mathematical Society, Providence 2009

45. Lewicka, M., Pakzad, M.R.: Scaling laws for non-Euclidean plates and the $W^{2,2}$ isometric immersions of Riemannian metrics. ESAIM Control Optim. Calc. Var. 17(4), $1158-1173,2011$

46. Marcellini, P.: Periodic solutions and homogenization of nonlinear variational problems. Ann. Mat. Pura Appl. 4(117), 139-152, 1978

47. MülleR, S.: Homogenization of nonconvex integral functionals and cellular elastic materials. Arch. Ration. Mech. Anal. 99(3), 189-212, 1987

48. Müller, S.: Homogenization of nonconvex integral functionals and cellular elastic materials. Arch. Ration. Mech. Anal. 99(3), 189-212, 1987

49. Müller, S.: Variational models for microstructure and phase transitions. In: HildeBRAndt, S., Struwe, M. (eds.) Calculus of Variations and Geometric Evolution Problems. Lecture Notes in Mathematics, vol. 1713, pp. 85-210. Springer, Berlin 1999

50. Müller, S., ScARdia, L., ZePPIERI, C.I.: Geometric rigidity for incompatible fields, and an application to strain-gradient plasticity. Indiana Univ. Math. J. 63(5), 1365-1396, 2014

51. Neukamm, S., SchäFfner, M.: Quantitative homogenization in nonlinear elasticity for small loads. Arch. Ration. Mech. Anal. 230(1), 343-396, 2018

52. Pratelli, A.: On the bi-Sobolev planar homeomorphisms and their approximation. Nonlinear Anal. 154, 258-268, 2017

53. ReŠEtNJAK, J.G.: Liouville's conformal mapping theorem under minimal regularity hypotheses. Sibirsk. Mat. Ž. 8, 835-840, 1967

54. Rudin, W.: Principles of Mathematical Analysis. International Series in Pure and Applied Mathematics, 3rd edn. McGraw-Hill, New York 1976

\author{
FABIAN Christowiak \\ Fakultät für Mathematik, \\ Universität Regensburg, \\ 93040 Regensburg \\ Germany.
}

e-mail: Fabian.Christowiak@mathematik.uni-regensburg.de

and

Carolin Kreisbeck

Mathematisch Instituut,

Universiteit Utrecht,

Postbus 80010, 3508 TA Utrecht

The Netherlands.

e-mail: c.kreisbeck@uu.nl

(Received August 30, 2018 / Accepted July 1, 2019)

Published online July 17, 2019

(C) The Author(s) (2019) 